\title{
Fast, Rate-Independent, Finite Element Implementation of a 3D Constrained Mixture Model of Soft Tissue Growth and Remodeling
}

\author{
Marcos Latorre ${ }^{\mathrm{a}, *}$, Jay D. Humphrey ${ }^{\mathrm{a}, \mathrm{b}}$ \\ ${ }^{a}$ Department of Biomedical Engineering \\ Yale University, New Haven, CT, USA \\ ${ }^{b}$ Vascular Biology and Therapeutics Program \\ Yale School of Medicine, New Haven, CT, USA
}

\begin{abstract}
Constrained mixture models of soft tissue growth and remodeling can simulate many evolving conditions in health as well as in disease and its treatment, but they can be computationally expensive. In this paper, we derive a new fast, robust finite element implementation based on a concept of mechanobiological equilibrium that yields fully resolved solutions and allows computation of quasi-equilibrated evolutions when imposed perturbations are slow relative to the adaptive process. We demonstrate quadratic convergence and verify the model via comparisons with semi-analytical solutions for arterial mechanics. We further examine the enlargement of aortic aneurysms for which we identify new mechanobiological insights into factors that affect the nearby non-aneurysmal segment as it responds to the changing mechanics within the diseased segment. Because this new 3D approach can be implemented within many existing finite element solvers, constrained mixture models of growth and remodeling can now be used more widely.
\end{abstract}

Keywords: growth, remodeling, constrained mixture, mechanobiology, artery

\section{Introduction}

A distinguishing feature of soft biological tissues is their ability to grow (change mass) and remodel (change microstructure) in response to diverse stimuli, often mechanical and chemical. Multiple approaches for mathematically modeling such growth and remodeling $(\mathrm{G} \& \mathrm{R})$ have proven useful in describing diverse situations for many different tissues [1-5]. Among these approaches, a constrained mixture model has proven particularly useful when there is a need to account for the different natural configurations, material properties, and rates of turnover of the individual constituents that define the tissue [6]. The classical (heredity integral-based) formulation of this mixture approach is computationally expensive, however, hence most implementations have focused on simple geometries (e.g., cylindrical bodies or axisymmetric membranes).

Herein, we exploit a recent concept of mechanobiologically equilibrated G\&R [7] and show computational advantages for illustrative cases that allow direct comparison with full constrained mixture solutions. We also extend prior kinematics to account for general three-dimensional G\&R with finite deformations and possible rotations. The proposed rate-independent framework can compute evolving homeostatic states efficiently by enforcing mechanical and mechanobiological equilibrium without the need to track the past history of deposition and removal, as in integral-based approaches, or to integrate evolution equations, as in rate-based approaches. We submit that this new 3D formulation, which can be implemented easily within existing finite element solvers though with a non-symmetric tangent stiffness

\footnotetext{
${ }^{*}$ Corresponding author

Email addresses: marcos.latorre@yale.edu (Marcos Latorre), jay.humphrey@yale.edu (Jay D. Humphrey)
} 
matrix, will enable fast, reliable finite element simulations of many G\&R problems while accounting for critical differences in the diverse constituents that characterize soft tissues.

\section{A mechanobiologically equilibrated constrained mixture model}

We first review local equations for mechanobiologically equilibrated mass fractions, deformation gradients, strain energy functions, and stresses, at constituent and mixture levels, which when complemented with an equilibrium value for a given stimulus function for mass production furnish a set of equations to compute fully resolved states at any material point and G\&R time $s$.

\subsection{Mechanobiological equilibrium}

Consider an in vivo loaded configuration $\kappa$ of a soft tissue that consists of a mixture of multiple constituents, in particular, various types of cells, extracellular matrix proteins, glycosaminoglycans, and abundant water. Because effects of internal solid-fluid interactions are negligible with respect to other characteristics exhibited by these tissues for applications of interest (e.g., ex vivo testing and in vivo behaviors during cyclic loading), we do not account explicitly for a solid-fluid mixture with momentum exchanges. Rather, the tissue is modeled as a constrained mixture of multiple hydrated solid constituents, with the hydrostatic pressure associated with interstitial water absorbed by a Lagrange multiplier that enforces transient incompressibility. In a continuum theory of constrained mixtures for G\&R [6], properties at a material point in configuration $\kappa$ are represented in a locally averaged sense, in terms of multiple structurally significant constituents $\alpha=1, \ldots, N$, to satisfy mass balance in spatial form

$$
\frac{\partial \rho^{\alpha}}{\partial s}+\operatorname{div}\left(\rho^{\alpha} \mathbf{v}^{\alpha}\right)=\bar{m}^{\alpha}
$$

where $\rho^{\alpha}$ is the homogenized, apparent mass density (mass of constituent $\alpha$ per unit current volume of mixture), $\mathbf{v}^{\alpha}$ the velocity (constrained to equal the velocity $\mathbf{v}$ of the mixture), and $\bar{m}^{\alpha}$ the net rate of mass density production or removal, which must be prescribed constitutively. Let $\bar{m}^{\alpha}$ be defined in terms of true rates of mass density production $m^{\alpha}>0$ and removal $n^{\alpha}>0$ as $\bar{m}^{\alpha}=m^{\alpha}-n^{\alpha}$, both of which must be prescribed constitutively. It proves useful to define a stimulus function $\Upsilon^{\alpha}=m^{\alpha} / n^{\alpha}>0$, which enhances $\left(\Upsilon^{\alpha}-1>0\right)$, reduces $\left(\Upsilon^{\alpha}-1<0\right)$, or balances $\left(\Upsilon^{\alpha}-1=0\right)$ mass production with respect to mass removal. Constitutively prescribing $m^{\alpha}$ and $n^{\alpha}$ is tantamount to prescribing $n^{\alpha}$ and $\Upsilon^{\alpha}$, which is often more convenient from a modeling perspective. Because $\operatorname{div}\left(\rho^{\alpha} \mathbf{v}^{\alpha}\right)=\rho^{\alpha} \operatorname{div} \mathbf{v}^{\alpha}+\mathbf{v}^{\alpha} \cdot \operatorname{grad} \rho^{\alpha}$, thus $\partial \rho^{\alpha} / \partial s+\mathbf{v}^{\alpha} \cdot \operatorname{grad} \rho^{\alpha}=\dot{\rho}^{\alpha}$, with $\dot{\rho}^{\alpha}$ the material time derivative of $\rho^{\alpha}$, and $\operatorname{div} \mathbf{v}^{\alpha}=\operatorname{div} \mathbf{v}=\dot{J} / J$, with $J=\operatorname{det} \mathbf{F}$ the Jacobian determinant of a deformation gradient $\mathbf{F}$ defined at the mixture level, which conveniently describes (measurable) deformations between an initial, original homeostatic configuration $\kappa_{o}$ and $\kappa$. Equation (1) can thus be written in terms of the referential mass density $\rho_{\mathrm{R}}^{\alpha}=J \rho^{\alpha}$ (defined per unit reference volume of mixture) as

$$
\frac{\dot{\rho}_{\mathrm{R}}^{\alpha}}{J} \equiv \dot{\rho}^{\alpha}+\rho^{\alpha} \frac{\dot{J}}{J}=m^{\alpha}-n^{\alpha}=n^{\alpha}\left(\Upsilon^{\alpha}-1\right),
$$

which generalizes rate equations for $\dot{\rho}_{\mathrm{R}}^{\alpha}$ obtained previously [7, 8] from an integral-based approach based on a first-order kinetic decay $n^{\alpha}=k^{\alpha} \rho^{\alpha}$, with $k^{\alpha}$ a rate parameter that defines the removal. Similarly, spatial linear momentum balance for constituent $\alpha$, with vanishing interactive forces among constituents due to their constrained motion, may be written as

$$
\rho^{\alpha} \dot{\mathbf{v}}^{\alpha}=\operatorname{div} \boldsymbol{\sigma}_{\mathrm{t}}^{\alpha}+\rho^{\alpha} \mathbf{b}^{\alpha}+\mathbf{p}_{\text {open }}^{\alpha}
$$

where $\boldsymbol{\sigma}_{\mathrm{t}}^{\alpha}$ is the total Cauchy stress tensor (i.e., including a contribution $\boldsymbol{\sigma}^{\alpha}$ derived from a strain energy function and others arising from kinematic constraints, such as incompressibility) for a constituent at the mixture level and $\mathbf{b}^{\alpha}$ is the constituent body force per unit current mass of constituent; additionally, 
$\mathbf{p}_{\text {open }}^{\alpha}$ represents the (excess) exchange of momentum not caused by the net exchange in mass $\bar{m}^{\alpha}$, which is typically zero in soft tissues.

Summation of mass (2) and momentum (3) balances over all constituents, with $\mathbf{v}^{\alpha}=\mathbf{v}$ and $\sum \mathbf{p}_{\mathrm{open}}^{\alpha} \simeq$ 0, yields the mixture relations

$$
\frac{\dot{\rho}_{\mathrm{R}}}{J} \equiv \dot{\rho}+\rho \frac{\dot{J}}{J}=m-n=\sum_{\alpha=1}^{N} n^{\alpha}\left(\Upsilon^{\alpha}-1\right)=\bar{m}
$$

and

$$
\rho \dot{\mathbf{v}}=\operatorname{div} \boldsymbol{\sigma}+\rho \mathbf{b}
$$

where $\rho_{\mathrm{R}}=\sum \rho_{\mathrm{R}}^{\alpha}=J \rho, m=\sum m^{\alpha}, n=\sum n^{\alpha}, \boldsymbol{\sigma}=\sum \boldsymbol{\sigma}_{\mathrm{t}}^{\alpha}$, and $\rho \mathbf{b}=\sum \rho^{\alpha} \mathbf{b}^{\alpha}$.

As in [7], and because $n^{\alpha}>0$, we observe from Eqs. (2) and (4) that a sufficient condition for a soft tissue to be in mechanobiological equilibrium (to preserve its mixture mass, composition, and properties) is

$$
\Upsilon^{\alpha} \equiv 1 \quad \forall \alpha \quad \Longrightarrow \quad \dot{\rho}_{\mathrm{R}}^{\alpha} \equiv 0 \quad \forall \alpha, \quad \dot{\rho}_{\mathrm{R}}=0 .
$$

Mechanical (static) equilibrium in a homeostatic state (denoted by subscript $h$ ) additionally requires, from Eq. (5),

$$
\mathbf{v}^{\alpha}=\mathbf{v} \equiv \mathbf{0} \quad \forall \alpha \quad \Longrightarrow \quad \mathbf{0}=\operatorname{div} \boldsymbol{\sigma}+\rho \mathbf{b}, \quad \mathbf{F} \equiv \mathbf{F}_{h}
$$

with $\mathbf{F}_{h}$ describing deformations between $\kappa_{o}$ and any homeostatic configuration $\kappa_{h}$, original or evolved (Fig. 1), which allows adaptive homeostasis [9]. Importantly, Eqs. (6) and (7) also approximate G\&R processes wherein the characteristic rate for adaptation is faster than the rate of change of the stimulation, thus yielding mechanobiologically quasi-equilibrated G\&R evolutions [10, 11].

\subsection{Mechanobiologically equilibrated mass fractions}

To generalize the present formulation, consider two types of load-bearing constituents $\alpha=\zeta \cup \xi$ within a soft tissue at G\&R time $s=0$ that evolve differently for $s>0$. Let constituents $\zeta=1, . ., N^{\zeta}$ not turnover, whereby their mass remains constant and their response can be described with (rateindependent) hyperelasticity. In contrast, let constituents $\xi=1, . ., N^{\xi}$ turnover continuously within extant matrix, thus contributing to local changes in mass and microstructure, with their response described with rate-independent G\&R. Respective initial mass fractions $\phi_{o}^{\zeta}$ and $\phi_{o}^{\xi}$ satisfy $\sum \phi_{o}^{\zeta}+\sum \phi_{o}^{\xi}=1$, with $N^{\zeta}+N^{\xi}=N$. Examples within the arterial wall are functional elastic fibers $(=\zeta)$, consisting of elastin and associated microfibrils, which are produced during the perinatal period and have a half-life of decades, and collagen fibers $(=\xi)$, which are produced continuously and have a half-life of weeks to months. Because differential production and removal of constituents $\xi$ contribute to changes in mass of the mixture, both types of constituents can present evolved mass fractions $\phi_{h}^{\zeta} \neq \phi_{o}^{\zeta}$ and $\phi_{h}^{\xi} \neq \phi_{o}^{\xi}$ at $\kappa_{h}$, satisfying $\sum \phi_{h}^{\zeta}+\sum \phi_{h}^{\xi}=1$, with

$$
\phi_{o}^{\alpha}=\frac{\rho_{o}^{\alpha}}{\rho}, \quad \phi_{h}^{\alpha}=\frac{\rho_{h}^{\alpha}}{\rho}
$$

for all constituents $\alpha=1, \ldots, N^{\zeta}+N^{\xi}$, where $\rho_{o}^{\alpha}$ and $\rho_{h}^{\alpha}$ are equilibrated mass densities (defined locally at $o$ or $h$, respectively), and $\rho$ is the actual mass density of the overall tissue (mixture), which we assume to be constant due to the highly hydrated states of all solid constituents.

\subsubsection{Constituents that do not turnover}

Because the mass of constituent $\zeta$ remains constant, a Piola transformation yields $\rho_{h}^{\zeta}\left(J_{h}\right)=\rho_{o}^{\zeta} / J_{h} \equiv$ $\rho_{\mathrm{R} h}^{\zeta} / J_{h}$, thus

$$
\phi_{h}^{\zeta}\left(J_{h}\right)=\frac{\phi_{o}^{\zeta}}{J_{h}}, \quad \zeta=1, \ldots, N^{\zeta}
$$




\subsubsection{Constituents that turnover}

To delineate differential changes in mass of constituents $\xi$ under mechanobiological equilibrium, one needs first to describe how they evolve with respect to each other under general G\&R. For example, without explicitly prescribing $n^{\xi}$ and $\Upsilon^{\xi}$ in Eq. (2), let all constituents $\xi$ respond to changes in stimuli with proportional out-of-equilibrium stimulus functions and mass-specific rates for removal: $\Upsilon^{\xi_{i}}-1=$ $\eta_{\Upsilon}^{i j}\left(\Upsilon^{\xi_{j}}-1\right)$ and $n^{\xi_{i}} / \rho^{\xi_{i}}=\eta_{\mathrm{q}}^{i j} n^{\xi_{j}} / \rho^{\xi_{j}}$ for $\xi_{i} \neq \xi_{j}=1, \ldots, N^{\xi}$, with $\eta_{\Upsilon}^{i j}$ and $\eta_{\mathrm{q}}^{i j}$ respective proportionality ratios. Then, from Eq. (2), local changes in constituent mass per respective unit mass satisfy

$$
\frac{\dot{\rho}_{\mathrm{R}}^{\xi_{i}}}{\rho_{\mathrm{R}}^{\xi_{i}}}=\eta^{i j} \frac{\dot{\rho}_{\mathrm{R}}^{\xi_{j}}}{\rho_{\mathrm{R}}^{\xi_{j}}}, \quad \xi_{i} \neq \xi_{j}=1, \ldots, N^{\xi}
$$

with $\eta^{i j}=\eta_{\mathrm{q}}^{i j} \eta_{\Upsilon}^{i j}$. Exact integration of Eqs. (10) from state $\kappa_{o}$ (for which $\rho_{\mathrm{R} o}^{\xi}=\rho_{o}^{\xi}$ ) to $\kappa_{h}$ yields the following $N^{\xi}-1$ independent relations among the mass fractions $\phi_{h}^{\xi}$

$$
\frac{J_{h} \phi_{h}^{\xi_{i}}}{\phi_{o}^{\xi_{i}}}=\left(\frac{J_{h} \phi_{h}^{\xi_{j}}}{\phi_{o}^{\xi_{j}}}\right)^{\eta^{i j}}, \quad \xi_{i} \neq \xi_{j}=1, \ldots, N^{\xi}
$$

where we used $\rho_{\mathrm{R} h}^{\xi}=J_{h} \rho_{h}^{\xi}$ and Eqs. (8), which are equivalent to those obtained in [7] between the two evolving constituents considered therein (smooth muscle cells " $m$ " and collagen fibers " $c$ ", with a single $\left.\eta=\eta_{\mathrm{q}} \eta_{\Upsilon}\right)$. Finally, the constraint $\sum \phi_{h}^{\alpha}=1$, with $\phi_{h}^{\zeta}\left(J_{h}\right)$ from Eq. (9), requires

$$
1=\sum_{\zeta=1}^{N^{\zeta}} \frac{\phi_{o}^{\zeta}}{J_{h}}+\sum_{\xi=1}^{N^{\xi}} \phi_{h}^{\xi}
$$

which, along with Eqs. (11), form a system of $N^{\xi}$ independent equations that provide implicit expressions for $\phi_{h}^{\xi}\left(J_{h}\right)$. If $\eta_{i j}=1 \forall\left\{\xi_{i}, \xi_{j}\right\}$, then Eq. (11) reduces to $\phi_{h}^{\xi_{i}} / \phi_{o}^{\xi_{i}} \equiv \phi_{h}^{\xi} / \phi_{o}^{\xi} \forall \xi_{i}$, and Eq. (12) to

$$
1=\sum_{\zeta=1}^{N^{\zeta}} \phi_{h}^{\zeta}\left(J_{h}\right)+\left(1-\sum_{\zeta=1}^{N^{\zeta}} \phi_{o}^{\zeta}\right) \frac{\phi_{h}^{\xi}}{\phi_{o}^{\xi}}
$$

where $\sum \phi_{o}^{\xi}=1-\sum \phi_{o}^{\zeta}$, which yields

$$
\frac{\phi_{h}^{\xi}\left(J_{h}\right)}{\phi_{o}^{\xi}}=\frac{1-\sum \phi_{h}^{\zeta}\left(J_{h}\right)}{1-\sum \phi_{o}^{\zeta}}, \quad \xi=1, \ldots, N^{\xi} .
$$

\subsection{Mechanobiologically equilibrated deformation gradients}

In a full constrained mixture theory, constituents are assumed to be deposited within the mixture at deposition time $\tau \leq s$ in intermediate configurations, relative to their own possibly evolving natural configurations $\kappa_{\mathrm{n}}^{\alpha}(\tau)$, via symmetric and volume-preserving deposition stretch tensors $\mathbf{G}^{\alpha}(\tau)$. Since the motion of each constituent, once deposited, equals that of the soft tissue, the deformation experienced by the material deposited at time $\tau$ that survives at $s$ is $\mathbf{F}_{\mathrm{n}(\tau)}^{\alpha}(s)=\mathbf{F}(s) \mathbf{F}^{-1}(\tau) \mathbf{G}^{\alpha}(\tau)[12]$. Because newly deposited constituents at time $\tau$ satisfy $\mathbf{F}_{\mathrm{n}(\tau)}^{\alpha}(\tau)=\mathbf{G}^{\alpha}(\tau)$, one notices (Appendix A) that $\kappa_{\mathrm{n}}^{\alpha}(\tau)$ can be interpreted as natural configurations that evolve with the configuration of the mixture $\kappa(\tau)$, with $\mathbf{G}^{\alpha}$ playing the role of a (spatial) left (pre)stretch tensor when referred to a rotated natural configuration $\kappa_{\mathrm{N}}^{\alpha}(\tau)$ that evolves while attached to the rotated configuration of the mixture $\kappa_{\mathrm{R}}(\tau)$. In that case, it proves convenient to let an associated deformation gradient

$$
\mathbf{F}_{\mathrm{N}(\tau)}^{\alpha}(s)=\mathbf{F}(s) \mathbf{F}^{-1}(\tau) \mathbf{F}_{\mathrm{G}}^{\alpha}(\tau)
$$


map line elements (fibers) from the rotated natural configuration $\kappa_{\mathrm{N}}^{\alpha}(\tau)$ to the current configuration $\kappa(s)$, where

$$
\mathbf{F}_{\mathrm{G}}^{\alpha}(\tau):=\mathbf{G}^{\alpha}(\tau) \mathbf{R}(\tau)=\mathbf{R}(\tau) \mathbf{G}_{\mathrm{N}}^{\alpha}(\tau)
$$

is a constituent-specific deposition tensor at $\tau$, with $\mathbf{R}$ the rotation tensor from a polar decomposition of $\mathbf{F}$, thus

$$
\mathbf{G}_{\mathrm{N}}^{\alpha}(\tau):=\mathbf{R}^{\mathrm{T}}(\tau) \mathbf{G}^{\alpha}(\tau) \mathbf{R}(\tau)
$$

is the associated (symmetric, volume-preserving) right (pre)stretch tensor defined in configuration $\kappa_{\mathrm{N}}^{\alpha}(\tau)$ (which is rotated with respect to the spatial configuration $\kappa_{\mathrm{n}}^{\alpha}(\tau)$ but unrotated with respect to the reference configuration of the mixture $\kappa(0))$. We then obtain mechanobiologically equilibrated deformation gradients for all constituents $\alpha$ from their respective equilibrated natural configurations to the equilibrated current configuration of the mixture $\kappa_{h}$, described by $\mathbf{F}(s)=\mathbf{F}_{h}$ (see Eq. (7) and Fig. 1).

\subsubsection{Constituents that do not turnover}

Constituents $\zeta$ are deposited and cross-linked prior to $s=0$; we account for their elastic response through mechanically equivalent deposition stretch tensors defined at the initial time $\mathbf{G}^{\zeta}(\tau=0)=\mathbf{G}^{\zeta}=$ const. Hence, their fictitious natural configurations $\kappa_{\mathrm{n}}^{\zeta}(0)=\kappa_{\mathrm{N}}^{\zeta}(0)$ do not evolve over time, but are fixed and attached to the reference configuration for the mixture $\kappa_{0}$. Equation (15) particularized to a mechanobiologically equilibrated state for which $\mathbf{F}(s)=\mathbf{F}_{h}$, with $\mathbf{F}(\tau=0)=\mathbf{I}$, yields (see Fig. 1)

$$
\mathbf{F}_{h}^{\zeta} \equiv \mathbf{F}_{\mathrm{N} h}^{\zeta}=\mathbf{F}_{\mathrm{n} h}^{\zeta}=\mathbf{F}_{h} \mathbf{G}^{\zeta}
$$

\subsubsection{Constituents that turnover}

Deposition stretches arise when synthetic cells act on the newly-secreted matrix via actomyosin activity [13], with magnitudes becoming constitutive parameters and so too the orientation of the new tissue when deposited $[12,14]$. We assume that the magnitude and orientation of $\mathbf{G}_{\mathrm{N}}^{\xi}(\tau)$ in Eqs. (15)-(17) remain constant $\forall \tau$, including mechanobiologically equilibrated evolutions $\forall s$, thus $\mathbf{G}_{\mathrm{N}}^{\xi}=$ const. Conversely, $\mathbf{G}_{h}^{\xi}=\mathbf{R}_{h} \mathbf{G}_{\mathrm{N}}^{\xi} \mathbf{R}_{h}^{\mathrm{T}} \neq \mathbf{c o n s t}$, in general, because rotations may arise as the vessel evolves. Equation (15) particularized for constituents that turnover within a homeostatic state, with $\mathbf{F}(\tau)=\mathbf{F}(s)=\mathbf{F}_{h}$ $\forall \tau$, yields (see Fig. 1)

$$
\mathbf{F}_{\mathrm{N} h}^{\xi}=\mathbf{F}_{h} \mathbf{F}_{h}^{-1} \mathbf{F}_{\mathrm{G} h}^{\xi}=\mathbf{F}_{\mathrm{G} h}^{\xi}=\mathbf{R}_{h} \mathbf{G}_{\mathrm{N}}^{\xi},
$$

which will prove useful when computing mechanobiologically equilibrated (rotated) Cauchy stresses subject to material frame indifference. Of course, more complex theories can include remodeling of deposition stretches in referential or spatial settings, with either their magnitude (e.g., via a fibrosis-driven maladaptation [15]) or alignment (e.g., via stress-, stretch-, or energy-driven reorientations [12, 16]) evolving over G\&R time scales, but we do not consider such cases here for simplicity.

\subsection{Mechanobiologically equilibrated strain energy}

\subsubsection{Constituents that do not turnover}

Consider strain energy functions $\hat{W}^{\zeta}$ per unit reference volume of their natural configurations $\kappa^{\zeta} \equiv$ $\kappa_{\mathrm{n}}^{\zeta}(0)=\kappa_{\mathrm{N}}^{\zeta}(0)$. Since constituents $\zeta$ are deposited within $\kappa_{o}=\kappa(0)$, associated contributions at the mixture level (per unit reference volume of mixture) are weighted directly by respective original volume fractions $\Phi_{o}^{\zeta}$ as $W_{\mathrm{R}}^{\zeta}=\Phi_{o}^{\zeta} \hat{W}^{\zeta}$. Noting that $\Phi_{o}^{\zeta}$ coincides with the mass fraction $\phi_{o}^{\zeta}$ for mixtures of constituents with the same mass density [7], as assumed herein,

$$
W_{\mathrm{R} h}^{\zeta}=\phi_{o}^{\zeta} \hat{W}_{h}^{\zeta}=\phi_{\mathrm{R} h}^{\zeta} \hat{W}_{h}^{\zeta}
$$

where $\phi_{\mathrm{R} h}^{\zeta}=\phi_{o}^{\zeta}$ and $\hat{W}_{h}^{\zeta}=\hat{W}^{\zeta}\left(\mathbf{C}_{h}^{\zeta}\right)$ depends on the equilibrated $\zeta$-constituent-specific right CauchyGreen tensor $\mathbf{C}_{h}^{\zeta}=\mathbf{F}_{h}^{\zeta \mathrm{T}} \mathbf{F}_{h}^{\zeta}=\mathbf{G}^{\zeta} \mathbf{C}_{h} \mathbf{G}^{\zeta}$, from Eq. (18), with $\mathbf{C}_{h}=\mathbf{F}_{h}^{\mathrm{T}} \mathbf{F}_{h}$ the right Cauchy-Green tensor for the equilibrated mixture. 


\subsubsection{Constituents that turnover}

Consider strain energy functions $\hat{W}^{\xi}$ per unit reference volume of their natural configurations $\kappa_{\mathrm{N}}^{\alpha} \equiv$ $\kappa_{\mathrm{N}}^{\alpha}(s)$, with $\kappa_{\mathrm{N}}^{\alpha}(s) \neq \kappa_{\mathrm{n}}^{\alpha}(s)$ in general. Since constituents $\xi$ are deposited within $\kappa_{h}=\kappa(s)$, contributions at the mixture level (per unit current volume of mixture) are weighted by evolved volume fractions $\Phi_{h}^{\xi}$ as $W^{\xi}=\Phi_{h}^{\xi} \hat{W}^{\xi}$, whereupon $W_{\mathrm{R}}^{\xi}=J_{h} W^{\xi}=J_{h} \Phi_{h}^{\xi} \hat{W}^{\xi}$. Noting that $\Phi_{h}^{\xi} \equiv \phi_{h}^{\xi}$,

$$
W_{\mathrm{R} h}^{\xi}=J_{h} \phi_{h}^{\xi} \hat{W}_{h}^{\xi}=\phi_{\mathrm{R} h}^{\xi} \hat{W}_{h}^{\xi}
$$

where $\phi_{\mathrm{R} h}^{\xi}=J_{h} \phi_{h}^{\xi}$ and $\hat{W}_{h}^{\xi}=\hat{W}^{\xi}\left(\mathbf{C}_{\mathrm{N} h}^{\xi}\right)$ depends on the equilibrated $\xi$-constituent-specific right CauchyGreen tensor $\mathbf{C}_{\mathrm{N} h}^{\xi}=\mathbf{F}_{h}^{\xi \mathrm{T}} \mathbf{F}_{h}^{\xi}=\left(\mathbf{G}_{\mathrm{N}}^{\xi}\right)^{2}$, from Eq. (19).

\subsubsection{Mixture}

Hence, we obtain the following rule-of-mixtures relation for $W_{\mathrm{R} h}$, defined per unit volume in $\kappa_{o}$, in terms of $\hat{W}_{h}^{\zeta}$, defined per unit volume in $\kappa^{\zeta}$, and $\hat{W}_{h}^{\xi}$, defined per unit volume in $\kappa_{\mathrm{N}}^{\xi}$

$$
W_{\mathrm{R} h}=\sum_{\zeta=1}^{N^{\zeta}} W_{\mathrm{R} h}^{\zeta}+\sum_{\xi=1}^{N^{\xi}} W_{\mathrm{R} h}^{\xi}=\sum_{\zeta=1}^{N^{\zeta}} \phi_{\mathrm{R} h}^{\zeta} \hat{W}_{h}^{\zeta}+\sum_{\xi=1}^{N^{\xi}} \phi_{\mathrm{R} h}^{\xi} \hat{W}_{h}^{\xi}=\sum_{\zeta=1}^{N^{\zeta}} \phi_{o}^{\zeta} \hat{W}_{h}^{\zeta}+J_{h} \sum_{\xi=1}^{N^{\xi}} \phi_{h}^{\xi} \hat{W}_{h}^{\xi}
$$

which, importantly, evolves consistent with both limits: $W_{\mathrm{R} h}\left(\sum \phi_{o}^{\zeta}=0\right)=J_{h} \sum \phi_{h}^{\xi} \hat{W}_{h}^{\xi}=J_{h} W_{h}$ (all constituents turn over) and $W_{\mathrm{R} h}\left(\sum \phi_{o}^{\zeta}=1\right)=\sum \phi_{o}^{\zeta} \hat{W}_{h}^{\zeta}=W_{h}$ (constituents do not turn over, thereby $J_{h} \equiv 1$ relates $W_{\mathrm{R}}$ and $W$ ). The mechanobiologically equilibrated strain energy for the mixture reads

$$
W_{\mathrm{R}}\left(\mathbf{C}_{h}\right)=\sum_{\zeta=1}^{N^{\zeta}} \phi_{o}^{\zeta} \hat{W}^{\zeta}\left(\mathbf{C}_{h}^{\zeta}\right)+J_{h} \sum_{\xi=1}^{N^{\xi}} \phi_{h}^{\xi}\left(J_{h}\right) \hat{W}^{\xi}\left(\mathbf{C}_{\mathrm{N} h}^{\xi}\right)
$$

with $\phi_{h}^{\xi}\left(J_{h}\right), \xi=1, \ldots, N^{\xi}$, from Section 2.2.2. This energy defines that stored by the mixture as a consequence of its current, pre-stretched homeostatic, in vivo state. An extension of Eq. (23) that allows computation of hyperelastic responses with respect to the configuration $\kappa_{h}$ is given in Appendix B.

\subsection{Mechanobiologically equilibrated stresses}

The second Piola-Kirchhoff stress for the mixture $\mathbf{S}$ during transient loading reads

$$
\begin{aligned}
\mathbf{S} & =\sum_{\zeta=1}^{N^{\zeta}} \mathbf{S}^{\zeta}+\sum_{\xi=1}^{N^{\xi}} \mathbf{S}^{\xi}-J_{h} p \mathbf{C}^{-1} \\
& =\sum_{\zeta=1}^{N^{\zeta}} \phi_{o}^{\zeta} \mathbf{G}^{\zeta} \hat{\mathbf{S}}^{\zeta}\left(\mathbf{C}^{\zeta}\right) \mathbf{G}^{\zeta}+J_{h} \sum_{\xi=1}^{N^{\xi}} \phi_{h}^{\xi} \mathbf{U}_{h}^{-1} \mathbf{G}_{\mathrm{N}}^{\xi} \hat{\mathbf{S}}_{\mathrm{N}}^{\xi}\left(\mathbf{C}_{\mathrm{N}}^{\xi}\right) \mathbf{G}_{\mathrm{N}}^{\xi} \mathbf{U}_{h}^{-1}-J_{h} p \mathbf{C}^{-1}
\end{aligned}
$$

with the appropriate $\mathbf{C}, \mathbf{C}^{\zeta}(\mathbf{C})$, and $\mathbf{C}_{\mathbf{N}}^{\xi}(\mathbf{C})$ given in Appendix B. Note, too, that the mixture-level Lagrange multiplier $p$ associates with the (intermittently imposed) constraint $J=J_{h}$, and

$$
\hat{\mathbf{S}}^{\zeta}\left(\mathbf{C}^{\zeta}\right)=2 \frac{\partial \hat{W}^{\zeta}\left(\mathbf{C}^{\zeta}\right)}{\partial \mathbf{C}^{\zeta}}, \quad \hat{\mathbf{S}}_{\mathrm{N}}^{\xi}\left(\mathbf{C}_{\mathrm{N}}^{\xi}\right)=2 \frac{\partial \hat{W}^{\xi}\left(\mathbf{C}_{\mathrm{N}}^{\xi}\right)}{\partial \mathbf{C}_{\mathrm{N}}^{\xi}}
$$

are second Piola-Kirchhoff stresses at the constituent level for both types of constituents. Straightforward particularization of these expressions to $\mathbf{F}=\mathbf{F}_{h}$ provides mechanobiologically equilibrated stresses at the current in vivo state. 


\subsubsection{Constituents that do not turnover}

$\mathbf{C}^{\zeta}$ particularizes to $\mathbf{C}_{h}^{\zeta}=\mathbf{G}^{\zeta} \mathbf{C}_{h} \mathbf{G}^{\zeta} \neq \mathbf{c o n s t}, \hat{\mathbf{S}}^{\zeta}$ to $\hat{\mathbf{S}}_{h}^{\zeta}=\hat{\mathbf{S}}^{\zeta}\left(\mathbf{C}_{h}^{\zeta}\right) \neq$ const (in general), and $\mathbf{S}^{\zeta}$ in Eq. (24) to

$$
\mathbf{S}_{h}^{\zeta}=\phi_{o}^{\zeta} \mathbf{G}^{\zeta} \hat{\mathbf{S}}_{h}^{\zeta} \mathbf{G}^{\zeta}
$$

Associated equilibrated Cauchy stresses read

$$
\boldsymbol{\sigma}_{h}^{\zeta}=\frac{1}{J_{h}} \mathbf{F}_{h} \mathbf{S}_{h}^{\zeta} \mathbf{F}_{h}^{\mathrm{T}}=\frac{\phi_{o}^{\zeta}}{J_{h}} \mathbf{F}_{h} \mathbf{G}^{\zeta} \hat{\mathbf{S}}_{h}^{\zeta} \mathbf{G}^{\zeta} \mathbf{F}_{h}^{\mathrm{T}}=\phi_{h}^{\zeta} \mathbf{F}_{h}^{\zeta} \hat{\mathbf{S}}_{h}^{\zeta} \mathbf{F}_{h}^{\zeta \mathrm{T}}
$$

where we used Eq. (18) (see Fig. 1).

\subsubsection{Constituents that turnover} to

$\mathbf{C}_{\mathrm{N}}^{\xi}$ particularizes to $\mathbf{C}_{\mathrm{N} h}^{\xi}=\mathbf{G}_{\mathrm{N}}^{\xi 2}=$ const, $\hat{\mathbf{S}}_{\mathrm{N}}^{\xi}$ to $\hat{\mathbf{S}}_{\mathrm{N} h}^{\xi}=\hat{\mathbf{S}}_{\mathrm{N}}^{\xi}\left(\mathbf{C}_{\mathrm{N} h}^{\xi}\right)=\mathbf{c o n s t}=\hat{\mathbf{S}}_{\mathrm{N}}^{\xi}$, and $\mathbf{S}^{\xi}$ in Eq.

$$
\mathbf{S}_{h}^{\xi}=J_{h} \phi_{h}^{\xi} \mathbf{U}_{h}^{-1} \mathbf{G}_{\mathrm{N}}^{\xi} \hat{\mathbf{S}}_{\mathrm{N}}^{\xi} \mathbf{G}_{\mathrm{N}}^{\xi} \mathbf{U}_{h}^{-1}
$$

Associated equilibrated Cauchy stresses read

$$
\boldsymbol{\sigma}_{h}^{\xi}=\frac{1}{J_{h}} \mathbf{F}_{h} \mathbf{S}_{h}^{\xi} \mathbf{F}_{h}^{\mathrm{T}}=\phi_{h}^{\xi} \mathbf{R}_{h} \mathbf{G}_{\mathrm{N}}^{\xi} \hat{\mathbf{S}}_{\mathrm{N}}^{\xi} \mathbf{G}_{\mathrm{N}}^{\xi} \mathbf{R}_{h}^{\mathrm{T}}=\phi_{h}^{\xi} \mathbf{F}_{\mathrm{N} h}^{\xi} \hat{\mathbf{S}}_{\mathrm{N}}^{\xi} \mathbf{F}_{\mathrm{N} h}^{\xi \mathrm{T}}=\phi_{h}^{\xi} \mathbf{R}_{h} \hat{\boldsymbol{\sigma}}_{\mathrm{N}}^{\xi} \mathbf{R}_{h}^{\mathrm{T}}
$$

where we used $\mathbf{F}_{h} \mathbf{U}_{h}^{-1}=\mathbf{R}_{h}$, Eq. (19) (see Fig. 1), and a rotated Cauchy stress tensor for constituent $\xi$ at the constituent level

$$
\hat{\boldsymbol{\sigma}}_{\mathrm{N}}^{\xi}=\mathbf{G}_{\mathrm{N}}^{\xi} \hat{\mathbf{S}}_{\mathrm{N}}^{\xi} \mathbf{G}_{\mathrm{N}}^{\xi}=\text { const }
$$

which remains constant during the mechanobiologically equilibrated evolutions considered here.

\subsubsection{Mixture}

Hence, at homeostatic states $\mathbf{F}=\mathbf{F}_{h}$, equilibrated second Piola-Kirchhoff stresses for the mixture are

$$
\mathbf{S}_{h}=\sum_{\zeta=1}^{N^{\zeta}} \mathbf{S}_{h}^{\zeta}+\sum_{\xi=1}^{N^{\xi}} \mathbf{S}_{h}^{\xi}-J_{h} p_{h} \mathbf{C}_{h}^{-1}
$$

with $\mathbf{S}_{h}^{\zeta}$ and $\mathbf{S}_{h}^{\xi}$ given in Eqs. (26) and (28), respectively. Associated Cauchy stresses, to be used in Eq. (7), specialize to the following evolved rule of mixtures

$$
\boldsymbol{\sigma}_{h}=\sum_{\alpha=1}^{N} \boldsymbol{\sigma}_{\mathrm{t} h}^{\alpha}=\sum_{\zeta=1}^{N^{\zeta}} \boldsymbol{\sigma}_{h}^{\zeta}+\sum_{\xi=1}^{N^{\xi}} \boldsymbol{\sigma}_{h}^{\xi}-p_{h} \mathbf{I}=\sum_{\zeta=1}^{N^{\zeta}} \phi_{h}^{\zeta} \mathbf{F}_{h}^{\zeta} \hat{\mathbf{S}}_{h}^{\zeta} \mathbf{F}_{h}^{\zeta \mathrm{T}}+\sum_{\xi=1}^{N^{\xi}} \phi_{h}^{\xi} \mathbf{F}_{\mathrm{N} h}^{\xi} \hat{\mathbf{S}}_{\mathrm{N} h}^{\xi} \mathbf{F}_{\mathrm{N} h}^{\xi \mathrm{T}}-p_{h} \mathbf{I}
$$

with $\boldsymbol{\sigma}_{h}^{\xi}$ given alternatively in terms of $\hat{\boldsymbol{\sigma}}_{\mathrm{N}}^{\xi}=$ const in Eq. (29). Note that $\boldsymbol{\sigma}_{\mathrm{th}}^{\alpha}$ in Eq. (3) includes $\boldsymbol{\sigma}_{h}^{\alpha}$, derived from the strain energy, and an associated reaction from the Lagrange multiplier $-p_{h} \mathbf{I}$.

\subsection{Mechanobiologically equilibrated stimulus function and Lagrange multiplier}

The hyperelastic stresses $\boldsymbol{\sigma}_{h}^{\zeta}$ in Eq. (32) are computed from the equilibrated deformation gradient $\mathbf{F}_{h}$, Jacobian-dependent mass fraction $\phi^{\zeta}\left(J_{h}\right)=\phi_{o}^{\zeta} / J_{h}$, and equilibrated stresses $\hat{\mathbf{S}}_{h}^{\zeta}=\hat{\mathbf{S}}^{\zeta}\left(\mathbf{C}_{h}^{\zeta}\right)$, in Eq. $(27)$; in contrast, $\boldsymbol{\sigma}_{h}^{\xi}$, in Eq. (29), are computed from the unique equilibrated rotation $\mathbf{R}_{h}$ from $\mathbf{F}_{h}$, Jacobiandependent mass fraction $\phi^{\xi}\left(J_{h}\right)$, in Section 2.2.2, and the constant tensor $\hat{\boldsymbol{\sigma}}_{\mathrm{N} h}^{\xi}$, in Eq. (30). Hence, only $p_{h}$ in Eq. (32) remains unknown, which one obtains by invoking mechanobiological equilibrium conditions in Eq. (6). 
Motivated by Fung's call for mass-and-structure growth-stress relations [17], and consistent with previous constrained mixture models, let $\xi$-constituent-specific stimulus functions $\Upsilon^{\xi}$ account for changes in mass production in response to cell-perceived changes in stress relative to homeostatic baseline values. In particular, among other possible equilibrated stimuli, let $\Upsilon_{h}^{\xi}$ depend on a set of invariants $\tilde{\sigma}_{h}^{i}$ of the total Cauchy stress tensor $\boldsymbol{\sigma}_{h}$, and perhaps structural tensors, that include the extent of $p_{h}$, that is $\tilde{\sigma}_{h}^{i}\left(p_{h}\right)$ from Eq. (32). Moreover, let the out-of-equilibrium stimulus functions $\Upsilon^{\xi}-1$ be proportional to each other, as in Section 2.2.2, so equilibrium conditions in Eq. (6) reduce $\forall \xi$ to a single nonlinear algebraic equation $\Upsilon_{h}=1$. Finally, let $\mathbf{S}_{h}^{x}:=\sum \mathbf{S}_{h}^{\alpha}=\sum \mathbf{S}_{h}^{\zeta}+\sum \mathbf{S}_{h}^{\xi}$ in Eq. (31) be the total "extra" [18], with superscript $x$, second Piola-Kirchhoff stresses, with associated Cauchy stresses $\boldsymbol{\sigma}_{h}^{x}=\frac{1}{J_{h}} \mathbf{F}_{h} \mathbf{S}_{h}^{x} \mathbf{F}_{h}^{\mathrm{T}}$ given in Eq. (32), which allows invariants $\tilde{\sigma}_{h}^{i}$ to be expressed in terms of scalar products involving the second-order tensors $\mathbf{C}_{h}, \mathbf{S}_{h}^{x}$, scalars $J_{h}, p_{h}$, and their couplings, that depend on $\mathbf{C}_{h}$ and $p_{h}$. Thus

$$
\Upsilon_{h}\left(\tilde{\sigma}_{h}^{1}\left(\mathbf{C}_{h}, p_{h}\right), \ldots, \tilde{\sigma}_{h}^{I}\left(\mathbf{C}_{h}, p_{h}\right)\right)=1 \Longrightarrow p_{h}\left(\mathbf{C}_{h}\right)
$$

which is a generally implicit relation that yields the (a priori unknown) volumetric contribution to stress at the mixture level in Eq. (32). As an important consequence, the mechanobiologically equilibrated stress field given by Eq. (32), complemented by (33), depends only on the current state of deformation and, hence, is path-independent. Nonetheless, similar to the stress field for a Cauchy elastic material [19], the mechanical work done by this mechanobiologically equilibrated stress field is, in general, pathdependent, which will have further implications regarding its constitutive tangent, as noted in Section 3. The associated material model is summarized in Box 1.

Example. For illustrative purposes and based on previous G\&R analyses of prototypical cylindrical arteries $[7,8,10]$, consider a linearized $\Upsilon_{h}\left(\tilde{\sigma}_{h}\right)$, with $\tilde{\sigma}_{h}=\sigma_{I h}=\operatorname{tr} \boldsymbol{\sigma}_{h}$ the first principal invariant of $\boldsymbol{\sigma}_{h}$, that is,

$$
\Upsilon_{h}\left(\sigma_{I h}\right)=1+K_{\sigma}\left(\frac{\sigma_{I h}-\sigma_{I o}}{\sigma_{I o}}\right)
$$

with $K_{\sigma}$ a gain parameter for mass production; hence, a possible flow-induced shear stress stimulus for G\&R is neglected here for simplicity (see Appendix C for possible accounts of this effect on different 3D computational frameworks). Thus, $\Upsilon_{h}=1$ yields $\sigma_{I h}=\sigma_{I o}$, or, in terms of volumetric (hydrostatic) components of stress $\sigma_{v}=\frac{1}{3} \operatorname{tr} \boldsymbol{\sigma}$

$$
\sigma_{v h}=\sigma_{v o}
$$

which thereby remains constant during mechanobiologically equilibrated G\&R, with its initial value $\sigma_{v o}=\frac{1}{3} \operatorname{tr} \sigma_{o}$ known from the reference state. This choice of stress stimulus, initially motivated to capture the biaxial nature of in vivo wall stresses during arterial G\&R adaptations (with the arterial wall subject to transmural pressure and axial stretch), relates closely to other choices in the literature where representative scalars of the volumetric stress contribution were identified as thermodynamic forces driving growth $[20,21]$. From Eq. $(32), \sigma_{v h}=\sum \sigma_{v h}^{\alpha}-p_{h}=\sigma_{v o}$, which yields $p_{h}$ as

$$
p_{h}\left(\mathbf{C}_{h}\right)=\sigma_{v h}^{x}-\sigma_{v o}=\frac{1}{3} \boldsymbol{\sigma}_{h}^{x}: \mathbf{I}-\sigma_{v o}=\frac{1}{3 J_{h}\left(\mathbf{C}_{h}\right)} \mathbf{S}_{h}^{x}\left(\mathbf{C}_{h}\right): \mathbf{C}_{h}-\sigma_{v o}
$$

and Cauchy stresses, from Eq. (32), decompose into deformation-dependent deviatoric and constant volumetric components

$$
\boldsymbol{\sigma}_{h}=\sum_{\alpha=1}^{N}\left(\boldsymbol{\sigma}_{h}^{\alpha}-\sigma_{v h}^{\alpha} \mathbf{I}\right)+\sigma_{v o} \mathbf{I} .
$$

Remark 1. For quasi-static incompressible hyperelastic behaviors, one generally satisfies mechanical equilibrium subject to boundary conditions and the incompressibility constraint $J=1$. This set of equations determines the Lagrange multiplier $p$ required to maintain incompressibility. In contrast, in 
the present G\&R framework, one solves mechanical equilibrium (Eq. (7)) subject to boundary conditions and the mechanobiological equilibrium constraint $\Upsilon_{h}=1$ (Eq. (6)). This set of equations determines the evolved homeostatic Lagrange multiplier $p_{h}$ consistent with a mechanobiologically equilibrated evolution. Note the difference between these two complementary rate-independent frameworks. The former satisfies the kinematic constraint $J=1$, with $p$ its associated stress-like Lagrange multiplier and $\sigma_{v}$ to be determined. The latter satisfies the stress-like constraint $\Upsilon_{h}=1$ (e.g., $\sigma_{v}$ constrained via Eq. (35)), with $p_{h}$ an associated stress-like consistency parameter and $J_{h}$ to be determined. Thus, $p_{h}$ does not enforce incompressibility during mechanobiologically equilibrated evolutions; it yet enables a smooth transition to stresses associated with intermittent hyperelastic responses superimposed at a given G\&R time $s=s_{h}$, for which an evolved constraint $J=J_{h}$ is to be considered, see Appendix B.

Remark 2. The pressure-sensitive stimulus function $\Upsilon$ in Eq. (33), which drives changes in volume via mass growth, resembles a pressure-sensitive yield function in rate-independent elastoplasticity, which triggers changes in volume via inelastic mechanisms but without mass exchange (such as for geomaterials, metallic foams, or filled polymers [22]). Since the present G\&R formulation contains other noticeable differences when compared to classical elastoplasticity (e.g., mixture theory describing different behaviors of different constituents, not a homogenized material; pre-stresses with evolving natural configurations; and a multiplicative decomposition without explicit consideration of an elastic gradient), elastic predictor / inelastic corrector integration schemes typically used in computational plasticity [23, 24] do not seem well suited for integrating the present rate-independent constrained-mixture equations. Rather, we solved this set of nonlinear equations exactly using a different stress-point resolution procedure (Box 1), whose consistent linearization for efficient finite element implementations is addressed next. Of course, other resolution (or integration) schemes and consistent continuum (or algorithmic) linearizations may apply for other rate-independent G\&R theories built upon different kinematic and / or constitutive assumptions (cf. [25]).

\section{Consistent linearization of the continuum theory}

In Section 2.5, we obtained (pre-)stresses for different constituents within a soft tissue in mechanobiological equilibrium by deriving, first, associated hyperelastic stresses during intermittent loading at a fixed G\&R time $s=s_{h}$, with $J=J_{h}$ and all constituent-specific natural configurations fixed, and, second, expressions specialized to a particular in vivo state $\mathbf{F}=\mathbf{F}_{h}$ along the hyperelastic response path. During an incremental mechanobiologically equilibrated evolution, the soft tissue and $\xi$-constituent-specific natural configurations, with associated equilibrated stresses, evolve following different paths over (longer) G\&R time scales [10]. We derive here tangent tensors for all load-bearing constituents and the mixture, consistent with a quasi-static G\&R evolution of this type. Subsequent consideration of an evolving homeostatic Lagrange multiplier $p_{h}$, arising from the mechanobiologically equilibrated constraint $\Upsilon_{h}=1$, completes the (exact) consistent linearization of the formulation. Importantly, since numerical approximations (arising, typically, from integration of evolution equations) are absent in this formulation, the continuum and algorithmic linearizations coincide; a similar situation arises in computational hyperelasticity but not computational plasticity, where linearization of the resulting discrete equations is essential to preserve asymptotic rates of quadratic convergence characteristic of Newton's methods [26].

\subsection{Constituents that do not turnover}

Equilibrated second Piola-Kirchhoff stresses in Eq. (26) can be written

$$
\mathbf{S}_{h}^{\zeta}=\phi_{o}^{\zeta} \mathbf{G}^{\zeta} \hat{\mathbf{S}}_{h}^{\zeta} \mathbf{G}^{\zeta}=\phi_{o}^{\zeta} \hat{\mathbf{S}}_{h}^{\zeta}: \mathbf{G}^{\zeta} \odot \mathbf{G}^{\zeta}
$$

where the symbol : implies the usual contraction of two indices and $\left(\mathbf{G}^{\zeta} \odot \mathbf{G}^{\zeta}\right)_{i j k l}=G_{i k}^{\zeta} G_{j l}^{\zeta}$, with $\mathbf{G}^{\zeta}$ symmetric. Since $\mathbf{G}^{\zeta}$ is constant, but $\mathbf{S}_{h}^{\zeta}=\hat{\mathbf{S}}^{\zeta}\left(\mathbf{C}_{h}^{\zeta}\right)$ depends on $\mathbf{C}_{h}$ through $\mathbf{C}_{h}^{\zeta}=\mathbf{G}^{\zeta} \mathbf{C}_{h} \mathbf{G}^{\zeta}$, the 
constitutive (hyperelastic) tangent at the mixture level reads

$$
\mathbb{C}_{h}^{\zeta}=2 \frac{\partial \mathbf{S}_{h}^{\zeta}}{\partial \mathbf{C}_{h}}=\phi_{o}^{\zeta} \mathbf{G}^{\zeta} \odot \mathbf{G}^{\zeta}: \hat{\mathbb{C}}_{h}^{\zeta}: \mathbf{G}^{\zeta} \odot \mathbf{G}^{\zeta}
$$

with $\hat{\mathbb{C}}_{h}^{\zeta}=2 \partial \hat{\mathbf{S}}_{h}^{\zeta} / \partial \mathbf{C}_{h}^{\zeta}$ the associated tangent at the constituent level.

\subsection{Constituents that turnover}

Equilibrated second Piola-Kirchhoff stresses in Eq. (28) can be written, using Eq. (30),

$$
\mathbf{S}_{h}^{\xi}=J_{h} \phi_{h}^{\xi} \mathbf{U}_{h}^{-1} \hat{\boldsymbol{\sigma}}_{\mathrm{N}}^{\xi} \mathbf{U}_{h}^{-1}=J_{h} \phi_{h}^{\xi} \hat{\boldsymbol{\sigma}}_{\mathrm{N}}^{\xi}: \mathbf{U}_{h}^{-1} \odot \mathbf{U}_{h}^{-1}
$$

Since $\hat{\boldsymbol{\sigma}}_{\mathrm{N}}^{\xi}=\mathbf{G}_{\mathrm{N}}^{\xi} \hat{\mathbf{S}}_{\mathrm{N}}^{\xi} \mathbf{G}_{\mathrm{N}}^{\xi}$ remains constant during mechanobiologically equilibrated evolutions, with natural configurations $\kappa_{\mathrm{N}}^{\xi}$ evolving accordingly, the (consistent) constitutive tangent at the mixture level reads

$$
\mathbb{C}_{h}^{\xi}=2 \frac{\partial \mathbf{S}_{h}^{\xi}}{\partial \mathbf{C}_{h}}=2 \mathbf{U}_{h}^{-1} \hat{\boldsymbol{\sigma}}_{\mathrm{N}}^{\xi} \mathbf{U}_{h}^{-1} \otimes \frac{\partial\left(J_{h} \phi_{h}^{\xi}\right)}{\partial \mathbf{C}_{h}}+2 J_{h} \phi_{h}^{\xi} \hat{\boldsymbol{\sigma}}_{\mathrm{N}}^{\xi}: \frac{\partial\left(\mathbf{U}_{h}^{-1} \odot \mathbf{U}_{h}^{-1}\right)}{\partial \mathbf{C}_{h}}
$$

with $\otimes$ the usual dyadic product and the fourth-order tensor $\hat{\boldsymbol{\sigma}}_{\mathrm{N}}^{\xi}: 2 \partial\left(\mathbf{U}_{h}^{-1} \odot \mathbf{U}_{h}^{-1}\right) / \partial \mathbf{C}_{h}=\mathbb{C}_{\hat{\boldsymbol{\sigma}}}^{\xi}$, resulting from the double contraction between a second-order tensor $\hat{\boldsymbol{\sigma}}_{\mathrm{N}}$ with a sixth-order tensor $2 \partial\left(\mathbf{U}_{h}^{-1} \odot\right.$ $\left.\mathbf{U}_{h}^{-1}\right) / \partial \mathbf{C}_{h}$, given in spectral decomposition form in Appendix D. Recalling from Section 2.2.2 that $J_{h} \phi_{h}^{\xi}=\phi_{\mathrm{R} h}^{\xi}$ depends exclusively on $J_{h}$, the chain rule on $\partial\left(J_{h} \phi_{h}^{\xi}\right) / \partial \mathbf{C}_{h}$, with $\partial J_{h} / \partial \mathbf{C}_{h}=\left(J_{h} / 2\right) \mathbf{C}_{h}^{-1}$, leads to

$$
\mathbb{C}_{h}^{\xi}=\frac{1}{\phi_{h}^{\xi}} \frac{d\left(J_{h} \phi_{h}^{\xi}\right)}{d J_{h}} \mathbf{S}_{h}^{\xi} \otimes \mathbf{C}_{h}^{-1}+J_{h} \phi_{h}^{\xi} \mathbb{C}_{\hat{\sigma}}^{\xi} .
$$

Note that Eqs. (11) and (12), each differentiated with respect to $J_{h}$, constitute a linear system of $N^{\xi}$ equations with $N^{\xi}$ unknowns $d\left(J_{h} \phi_{h}^{\xi}\right) / d J_{h}$, which can be solved explicitly. For example, if $\eta_{i j}=1$ $\forall\left\{\xi_{i}, \xi_{j}\right\}$, differentiation of the explicit result in Eq. (14) yields

$$
\frac{d\left(J_{h} \phi_{h}^{\xi}\right)}{d J_{h}}=\frac{\phi_{o}^{\xi}}{1-\sum \phi_{o}^{\zeta}}, \quad \xi=1, \ldots, N^{\xi} .
$$

\subsection{Lagrange multiplier contribution}

The term $\mathbf{S}_{h}^{p}:=-J_{h} p_{h} \mathbf{C}_{h}^{-1}$ in Eq. (31) evolves consistent with the requirement in Eq. (33), that is $\dot{\Upsilon}_{h} \equiv 0$, which defines $p_{h}\left(\mathbf{C}_{h}\right)$ constitutively during mechanobiological equilibrium and enables computation of a consistent tangent tensor

$$
\mathbb{C}_{h}^{p}=2 \frac{\partial \mathbf{S}_{h}^{p}}{\partial \mathbf{C}_{h}}=-2 \mathbf{C}_{h}^{-1} \otimes \frac{\partial\left(J_{h} p_{h}\right)}{\partial \mathbf{C}_{h}}-2 J_{h} p_{h} \frac{\partial \mathbf{C}_{h}^{-1}}{\partial \mathbf{C}_{h}} .
$$

\subsection{Mixture}

Using Eq. (31), the referential tangent tensor for the mixture is

$$
\mathbb{C}_{h}=2 \frac{\partial \mathbf{S}_{h}}{\partial \mathbf{C}_{h}}=\sum_{\zeta=1}^{N^{\zeta}} \mathbb{C}_{h}^{\zeta}+\sum_{\xi=1}^{N^{\xi}} \mathbb{C}_{h}^{\xi}+\mathbb{C}_{h}^{p}=\sum_{\alpha=1}^{N} \mathbb{C}_{h}^{\alpha}+\mathbb{C}_{h}^{p}=\mathbb{C}_{h}^{x}+\mathbb{C}_{h}^{p}
$$

where all contributions are given above (Eqs. (39), (42), (44)). Its associated spatial tangent is given by the push-forward operation, from $\kappa_{o}$ to $\kappa_{h}$,

$$
\mathbb{C}_{h}=\frac{1}{J_{h}} \mathbf{F}_{h} \odot \mathbf{F}_{h}: \mathbb{C}_{h}: \mathbf{F}_{h}^{\mathrm{T}} \odot \mathbf{F}_{h}^{\mathrm{T}} .
$$


Linearization of the material model is summarized in Box 2.

Example. (Cont.) Equation (36), expressed in terms of mixture-level Lagrangian deformations and stresses, yields $\mathbf{S}_{h}^{p}:=-J_{h} p_{h} \mathbf{C}_{h}^{-1}=\left(J_{h} \sigma_{v o}-\frac{1}{3} \mathbf{S}_{h}^{x}: \mathbf{C}_{h}\right) \mathbf{C}_{h}^{-1}$ in Eq. (31). From Eq. (44)

$$
\mathbb{C}_{h}^{p}=2 \frac{\partial \mathbf{S}_{h}^{p}}{\partial \mathbf{C}_{h}}=2 \sigma_{v o} \mathbf{C}_{h}^{-1} \otimes \frac{\partial J_{h}}{\partial \mathbf{C}_{h}}-\frac{2}{3} \mathbf{C}_{h}^{-1} \otimes \frac{\partial\left(\mathbf{S}_{h}^{x}: \mathbf{C}_{h}\right)}{\partial \mathbf{C}_{h}}-2 J_{h} p_{h} \frac{\partial \mathbf{C}_{h}^{-1}}{\partial \mathbf{C}_{h}}
$$

which, with $\partial J_{h} / \partial \mathbf{C}_{h}=\left(J_{h} / 2\right) \mathbf{C}_{h}^{-1}, \mathbb{C}_{h}^{x}:=\sum \mathbb{C}_{h}^{\alpha}$ from Section 3.1 and 3.2 , and $\left(\partial \mathbf{C}^{-1} / \partial \mathbf{C}\right)_{i j k l}:=$ $-\left(\mathbf{C}^{-1} \odot \mathbf{C}^{-1}\right)_{i j k l}=-\frac{1}{2}\left(C_{i k}^{-1} C_{j l}^{-1}+C_{i l}^{-1} C_{j k}^{-1}\right)$, finally yields

$$
\mathbb{C}_{h}^{p}=J_{h} \sigma_{v o} \mathbf{C}_{h}^{-1} \otimes \mathbf{C}_{h}^{-1}-\frac{1}{3} \mathbf{C}_{h}^{-1} \otimes \mathbf{C}_{h}: \mathbb{C}_{h}^{x}-\frac{2}{3} \mathbf{C}_{h}^{-1} \otimes \mathbf{S}_{h}^{x}+2 J_{h} p_{h} \mathbf{C}_{h}^{-1} \odot \mathbf{C}_{h}^{-1}
$$

Remark 3. The referential tangent tensor in Eq. (45), with Cartesian components $\left(\mathbb{C}_{h}\right)_{i j k l}$, includes different contributions that lack the major symmetry $i j k l \leftrightarrow k l i j$, in general. First, the tensor $\mathbb{C}_{h}^{\xi}$ in Eq. (42) was derived from mechanobiological equilibrium considerations at the constituent level, that is $\hat{\boldsymbol{\sigma}}_{\mathrm{N}}^{\xi}=\mathbf{G}_{\mathrm{N}}^{\xi} \hat{\mathbf{S}}_{\mathrm{N}}^{\xi} \mathbf{G}_{\mathrm{N}}^{\xi}=$ const in Eq. (40), so its symmetry is not guaranteed. This is in contrast with the tensor $\mathbb{C}_{h}^{\zeta}$ in Eq. (39), which derives from a hyperelastic potential, and hence is symmetric, even during G\&R evolutions. Second, linearization of the consistency parameter $p_{h}$ in $\mathbf{S}_{h}^{p}=-J_{h} p_{h} \mathbf{C}_{h}^{-1}$ may yield additional contributions lacking major symmetry, for example, the second and third terms in the right-hand side of Eq. (48), because the extra stresses $\mathbf{S}_{h}^{x}$ in Eq. (36) are not purely deviatoric, in general. This is in contrast with the first and fourth terms in Eq. (48), which are symmetric. All these non-symmetric tensors (cf. previous expressions and Appendix D as well) preserve the minor symmetries $i j k l \leftrightarrow j i k l \leftrightarrow j i l k \leftrightarrow i j l k$, which allows one to store their components for computational analyses using $6 \times 6$ matrices. This was not an issue for illustrative computations below, which required a nonsymmetric finite element formulation consistent with the application of follower forces (pressure) that change direction as a body deforms (cf., for example, [27, 28]). Other constitutive formulations based on internal variables, such as finite kinematic growth [21], experimentally motivated non-associative plasticity [22], or fully (elastic and viscous) anisotropic finite strain nonlinear viscoelasticity [29], may similarly lead to non-symmetric consistent tangents for finite element implementations.

\section{Original (loaded, in vivo) homeostatic state}

The formulation outlined in Boxes 1 and 2 is intended to yield quasi-static G\&R evolutions of threedimensional solids under continuous states of mechanobiological equilibrium. The formulation is defined locally at arbitrary material points and, hence, can be used to solve general boundary value problems employing standard finite element procedures. Inasmuch as $\Upsilon_{h}$ depends on changes in stress relative to baseline values, one needs to pre-compute the original in vivo state, that is, an initially loaded state under mechanical and mechanobiological equilibrium. If the soft tissue behaves elastically under transient loads, then we can compute this initial state, subject to initial loads, by departing from a given initial geometry, close to the one at G\&R time $s=0$, and solving a boundary value problem with a nearly incompressible hyperelastic response. The (pre)stress computed at each material point after this initial, required calculation serves as a reference for the subsequent G\&R computations by means of the specified equilibrium condition in $\Upsilon_{h}=1$, as, for example, the local preservation of volumetric Cauchy stresses at the mixture level specified in Eq. (35).

Hence, consider the stresses derived in Appendix B and particularized to the initial in vivo state $\kappa_{o}$, see Eq. (74), with $p$ an actual Lagrange multiplier associated with the incompressibility constraint $J=\operatorname{det} \mathbf{F}=1$ (i.e., at G\&R time $s=0$ ). In finite element implementations, however, one usually imposes near incompressibility through a penalty volumetric strain energy function $U(J)$ (with an associated 
bulk modulus far exceeding the shear modulus), from which $-p(J)$ is approximated by $d U(J) / d J$, with $J \simeq 1$. For convenience, we consider a penalty function $U(\ln J)$ and approximate $-J p(J)$ in Eq. (74) as $d U(\ln J) / d \ln J=: U^{\prime}(\ln J)$, whereupon

$$
\mathbf{S}=\sum_{\zeta=1}^{N^{\zeta}} \phi_{o}^{\zeta} \mathbf{G}^{\zeta} \hat{\mathbf{S}}^{\zeta}\left(\mathbf{C}^{\zeta}\right) \mathbf{G}^{\zeta}+\sum_{\xi=1}^{N^{\xi}} \phi_{o}^{\xi} \mathbf{G}_{\mathrm{N}}^{\xi} \hat{\mathbf{S}}_{\mathrm{N}}^{\xi}\left(\mathbf{C}_{\mathrm{N}}^{\xi}\right) \mathbf{G}_{\mathrm{N}}^{\xi}+U^{\prime}(\ln J) \mathbf{C}^{-1}
$$

where $\hat{\mathbf{S}}^{\zeta}\left(\mathbf{C}^{\zeta}\right)$ and $\hat{\mathbf{S}}_{\mathrm{N}}^{\xi}\left(\mathbf{C}_{\mathrm{N}}^{\xi}\right)$ are computed from respective constituent-specific strain energy functions, see Eq. (72), with $\mathbf{C}^{\zeta}=\mathbf{F}^{\zeta \mathrm{T}} \mathbf{F}^{\zeta}$ and $\mathbf{C}_{\mathrm{N}}^{\xi}=\mathbf{F}^{\xi \mathrm{T}} \mathbf{F}^{\xi}$ along with $\mathbf{F}^{\zeta}=\mathbf{F} \mathbf{G}^{\zeta}$ and $\mathbf{F}^{\xi}=\mathbf{F} \mathbf{G}_{\mathrm{N}}^{\xi}$. This expression for stresses is consistent with a constrained mixture theory for hyperelastic soft tissues with reference configuration $\kappa_{o}$ [30]. The constitutive tangent $\mathbb{C}=2 \partial \mathbf{S} / \partial \mathbf{C}$ during this initial, elastic pre-loading step reads

$$
\begin{aligned}
\mathbb{C} & =\sum_{\zeta=1}^{N^{\zeta}} \phi_{o}^{\zeta} \mathbf{G}^{\zeta} \odot \mathbf{G}^{\zeta}: \hat{\mathbb{C}}^{\zeta}\left(\mathbf{C}^{\zeta}\right): \mathbf{G}^{\zeta} \odot \mathbf{G}^{\zeta} \\
& +\sum_{\xi=1}^{N^{\xi}} \phi_{o}^{\xi} \mathbf{G}_{\mathrm{N}}^{\xi} \odot \mathbf{G}_{\mathrm{N}}^{\xi}: \hat{\mathbb{C}}_{\mathrm{N}}^{\xi}\left(\mathbf{C}_{\mathrm{N}}^{\xi}\right): \mathbf{G}_{\mathrm{N}}^{\xi} \odot \mathbf{G}_{\mathrm{N}}^{\xi} \\
& +U^{\prime \prime}(\ln J) \mathbf{C}^{-1} \otimes \mathbf{C}^{-1}-2 U^{\prime}(\ln J) \mathbf{C}^{-1} \odot \mathbf{C}^{-1}
\end{aligned}
$$

which can be implemented readily in a material subroutine for finite element analyses.

A step-by-step algorithm, including computations of associated stress and tangent moduli during both stages of our formulation (i.e., an initial pre-stressed state and subsequent equilibrated G\&R evolution), is summarized in Box 3.

\section{Illustrative numerical examples}

Consider particular relations that have proven useful in describing vascular G\&R, particularly for aneurysmal enlargement [12], cerebral vasospasm [31], aortic maladaptation in hypertension [15], and even the in vivo development of tissue engineered constructs [32]. A "four-fiber family" model includes three contributions: a stored energy function for an amorphous elastin-dominated matrix $(\zeta \equiv e)$

$$
\hat{W}^{e}=\frac{c^{e}}{2}\left(\mathbf{C}^{e}: \mathbf{I}-3\right)
$$

where $c^{e}$ is a shear modulus, plus circumferentially oriented passive smooth muscle $(\xi \equiv m)$ and circumferentially, axially, and diagonally oriented (with angle $\alpha_{0}$ with respect to the axial direction) collagen fibers $(\xi \equiv c)$ described by

$$
\hat{W}^{\xi}=\frac{c_{1}^{\xi}}{4 c_{2}^{\xi}}\left(\exp \left(c_{2}^{\xi}\left(\mathbf{C}_{\mathrm{N}}^{\xi}: \mathbf{a}_{\mathrm{N}}^{\xi} \otimes \mathbf{a}_{\mathrm{N}}^{\xi}-1\right)^{2}\right)-1\right)
$$

with $c_{1}^{\xi}$ and $c_{2}^{\xi}$ material parameters and $\mathbf{a}_{\mathrm{N}}^{\xi}$ unit vectors along fiber orientations in $\kappa_{\mathrm{N}}^{\xi}$. Effects of other constituents (such as proteoglycans) are captured phenomenologically via regression-based fits to data [15]. In addition, removal of constituents $\xi=m, c$ is governed via a first-order type of kinetic decay, with $k^{\xi}$ rate parameters [7]. Finally, recall that net mass production is given by Eq. (4) via the removal and stimulus functions, here driven quasi-statically through Eq. (6). Table 1 lists all needed geometric and material parameters for an illustrative mouse aorta, noting that arteries tend to preserve their overall mass density $\rho=1050 \mathrm{~kg} / \mathrm{m}^{3}$. 
We implemented the present rate-independent constrained-mixture framework for G\&R (Boxes 1 to 3) as a user material plugin in the open source software FEBio [33]. Because there is no need for local integration to update internal variables between incremental steps or storing long-term history-dependent variables, the implementation is straightforward and, from a numerical viewpoint, resembles that for a hyperelastic material (except for the non-symmetric consistent tangent and the need to solve numerically a system of equations for the Jacobian-dependent mass fractions $\left.\phi^{\xi}(J)\right)$. Solutions proceed in two stages. Stage I is not particular to the present formulation for G\&R, but to any formulation that requires a prestressed initial configuration, such as hyperelastic [34] or G\&R [35] formulations. Also note that mixture volumetric and constituent stresses are only stored once, at the end of Stage I, hence these initially computed variables play the role of (local) material parameters for Stage II. Importantly, however, the current version of FEBio used here (2.8.2) did not support materials with tangents having only minor symmetries, hence we added a new class of matrices and associated algebraic operators. In addition, some procedures within the FEBio source code were extended to handle non-symmetric constitutive tangents (i.e., an output from our material plugin) and FEBio was re-compiled accordingly.

\subsection{Computation of the original homeostatic state (Stage I)}

We compute here an original in vivo homeostatic state that will serve as the reference for all subsequent numerical simulations. Hence, consider an initially cylindrical arterial segment with inner radius $a_{o}$, thickness $h_{o}$, and length $l_{o}$ (Table 1) as a 3D finite element (FE) geometry (shown partially in Figure 2(a)). We fix axial displacements at both ends, with rigid body motions suppressed. As an external (pre-)load, we apply an in vivo value of blood pressure on the inner surface of the cylinder. Using a simple analytical (uniform) solution, see Appendix E or [7], one finds an in vivo pressure $P_{o}=13.98 \mathrm{kPa}=104.9 \mathrm{mmHg}$ consistent with model parameters in Table 1, as well as an original homeostatic Lagrange multiplier $p_{o}=10.21 \mathrm{kPa}$. Hence, consistent with the concept of prestretch (and associated prestress), we define a volumetric function $U\left(\ln \left(J J_{G}\right)\right)=\frac{1}{2} \kappa\left(\ln \left(J J_{G}\right)\right)^{2}$, with $\kappa=10^{3} c^{e}$ a (penalty) bulk modulus and $J_{G}$ a pre-Jacobian satisfying $U^{\prime}\left(\ln J_{G}\right)=\kappa \ln J_{G}=-p_{o}$, that is, $J_{G}=\exp \left(-p_{o} / \kappa\right)$ for $J=1$. With this, local stresses computed by Eq. (49) during the pre-loading stage are numerically close to those given analytically, the main difference being that the FE solution is appropriately non-uniform through the wall thickness. Only one static load step (with two-to-three global Newton-Raphson iterations) was needed to find associated equilibrium solutions (to machine precision) for the preload considered using different FE meshes. In any case, the computed equilibrium displacements for Stage I in Box 3 were negligible with respect to any characteristic length of the geometrical model, with $\mathbf{F}=\mathbf{F}_{o} \simeq \mathbf{I}$ and $J=J_{o} \simeq 1$ as desired; compare initial ( $a$, input) and computed ( $b$, output) cross-sections in Figure 2. Furthermore, note that we do not prescribe an in vivo axial stretch $\lambda_{z}^{\text {iv }}>1$ on this geometry (as one would do experimentally given an excised, unloaded vessel) because the computed solution implicitly includes an in vivo axial force (born by the axial constraints) consistent with the deposition stretches and associated stresses, in Table 1 , which in turn allows us to refer deformations to this loaded configuration (i.e., $\lambda_{z}=\lambda_{z o}=1$ refers to the original homeostatic state).

We next conducted a mesh convergence study to determine an appropriate description of in vivo through-the-thickness stresses (3). After using different types of finite elements and discretizations, and because of the presence of pre-stresses (that tend to homogenize the stress field across the wall) and a relatively low thickness-to-radius ratio in the mouse aorta, we found that four displacement-based hexahedral elements give an accurate through-the-thickness solution for the present unilayered model. Figure $2(b)$ shows associated in vivo volumetric Cauchy pre-stresses $\sigma_{v o}=(1 / 3) \operatorname{tr} \sigma_{o}$. This discretization is consistent with the one for displacements adopted in [36], where twelve mixed elements, linear in displacements and constant in pressure, were used to discretize three layers through the wall. As we explain below, volumetric locking is not expected during our quasi-static computations of mechanobiologically equilibrated G\&R during Stage II, hence, mixed formulations with additional interpolations of pressure at the element level are not needed. 
After computing this equilibrium solution, we stored total volumetric stresses for the mixture $\sigma_{v o}=$ $\left(1 / 3 J_{o}\right) \mathbf{S}_{o}: \mathbf{C}_{o}$ and rotated Cauchy stresses for smooth muscle and collagen families $\hat{\sigma}_{\mathrm{N}}^{\xi}=\left(1 / J_{o} \phi_{o}^{\xi}\right) \mathbf{U}_{o} \mathbf{S}_{o}^{\xi} \mathbf{U}_{o}$ at integration points, which were needed (as reference variables) subsequently to compute mechanobiologically equilibrated G\&R evolutions (Box 3). Note that we must compute these local values consistent with the non-uniform FE solution obtained, hence incorporating the extent of $\mathbf{F}_{o} \simeq \mathbf{I}$, rather than from exact theoretical expressions (e.g., Eq. (30)) consistent with the exact reference limit $\mathbf{F}_{o}=\mathbf{I}$. Thus, in practice, one needs to store $\mathbf{F}_{o}$ and then compute G\&R deformations during Stage II via $\mathbf{F}_{h}=\mathbf{F} \mathbf{F}_{o}^{-1}$ and $J_{h}=J J_{o}^{-1}$. This ensures a smooth numerical transition between Stages I and II, that is, when $\mathbf{F}=\mathbf{F}_{o}$ (i.e., $\left.\mathbf{F}_{h}\right|_{I \rightarrow I I}=\mathbf{F}_{o} \mathbf{F}_{o}^{-1}=\mathbf{I}$ and $\left.J_{h}\right|_{I \rightarrow I I}=J_{o} J_{o}^{-1}=1$, whereupon G\&R starts right when Stage II does).

\subsection{Equilibrated GERR response (Stage II) to increase in pressure, flow rate, or axial stretch}

We verify here that mechanobiologically equilibrated responses computed for cylindrical geometries (that remain cylindrical) with the proposed FE formulation agree with responses computed with our previous theoretical formulation for thin-walled cylindrical arteries [7]. We note that the analytical rate-independent formulation was already verified and validated against a full integral heredity-based constrained model in [7] for long-term equilibrium solutions, and in [10] for slow loading relative to G\&R.

For uniform material properties, the computed solutions are uniform in the circumferential and axial (in-plane) directions, but non-uniform along the radial direction consistent with different traction (pressure) conditions on the inner and outer boundaries. As explained in Appendix C, we can include a uniform flow-induced shear stress stimulus for mass production in the equilibrated function $\Upsilon_{h}$ in Eq. (33); that is, we can include mechanobiological effects of a shear stress that is negligible mechanically (five orders of magnitude smaller [18]). Consideration of this augmented function will let us compute, first, pressure- and/or axial stretch-induced G\&R responses for different intramural-to-shear stress gain ratios for mass production, and, second, flow-induced G\&R responses. It will also let us compare FE outcomes against their corresponding analytical solutions, see Appendix $\mathrm{C}$ and Appendix E. Consistent with an axially uniform solution, we employ a single element along the axial direction and report deformations and band plots for a representative cross section.

Figure 2 also shows deformed cross-sections and contour plots of volumetric stress obtained for $P_{h}=1.6 P_{o}=22.38 \mathrm{kPa}$ for two ratios of gain parameter, $K_{\tau} / K_{\sigma}=0$ (i.e., vanishing shear stress stimulus for mass production; mesh $c$ ) and $K_{\tau} / K_{\sigma}=1$ (i.e., both intramural and shear stress stimuli for mass production; mesh $d$ ). Figure 4 additionally shows associated pressure-dependent evolutions for global geometric parameters from the FE simulation (symbols) such as normalized inner radius (panel $a)$ and thickness $(b)$, as well as local variables at the mid-thickness such as volume ratio $(c)$, and circumferential $(d)$, axial $(e)$, and volumetric $(f)$ stresses, all compared to results computed with the uniform semi-analytical cylindrical model [7] (equivalently, Appendix E), which show excellent agreement. Note, however, the remarkably different responses that the mechanobiologically equilibrated models predict for $K_{\tau} / K_{\sigma}=0$ versus 1 , which may be understood by examining the associated stimulus function $\Upsilon_{h}$.

For $K_{\tau} / K_{\sigma}=0$, the constraint $\Upsilon_{h}=1$ implies preservation of volumetric stresses $\sigma_{v h}=\sigma_{v o}$ (recall Eqs. (35) or (37)), which the FE simulation satisfies locally at each radial location (Fig. 2) and, in particular, at the mid-thickness (Fig. 4). Albeit shown at only the mid-thickness, circumferential and axial Cauchy stresses remain nearly constant during this quasi-static G\&R evolution. Yet, this restoration of stress via mass growth arises from increases in inner radius (circumferential stretch) and thickness (radial stretch), and thus associated volume (Jacobian), which depart from the theoretical mechano-adaptive limits $a_{h} / a_{o} \rightarrow \epsilon_{h}^{1 / 3}=1$ and $h_{h} / h_{o} \rightarrow \gamma_{h} \epsilon_{h}^{1 / 3}=1.6$ (when $\epsilon_{h}=Q_{h} / Q_{o}=1$ and $\gamma_{h}=P_{h} / P_{o}=1.6$, with $Q$ blood flow rate), cf. [18]. Indeed, the analytical model predicts a marked growth response $\left(a_{h} / a_{o} \rightarrow \infty\right.$ and $\left.h_{h} / h_{o} \rightarrow \infty\right)$ for $K_{\tau} / K_{\sigma}=0$ and $P_{h} \rightarrow 1.95 P_{o}$, which suggests a static mechanobiological instability (or unbounded critical point) against increases in pressure, cf. [8]. Importantly, when accounting for the radially non-uniform distributions of stress / stretch, this instability developed earlier at the outer than at the inner layer of elements, which caused the FE simulation to diverge before reaching the theoretical blow-up pressure $1.95 P_{o}$ predicted by the uniform formulation. 
This finding suggests that full 3D simulations can be needed even for thin-walled arteries. It also reminds us of the need to account for all mechanobiological stimuli (endothelial cell shear and interstitial cell normal stresses) even when one component of stress is negligible mechanically.

In particular, for $K_{\tau} / K_{\sigma}=1$, the constraint $\Upsilon_{h}=1$ introduces an additional restriction on changes in flow-induced wall shear stress $\tau_{w h}$, which must offset changes in volumetric stress $\sigma_{v h}$ (recall Eq. (79)). For example, for the idealized wall shear stress expression $\tau_{w}=4 \mu Q / \pi a^{3}$ (with $\mu$ the blood viscosity) that we employ, a restriction on $\tau_{w h}$ with preserved cardiac output controls luminal dilation, which in turn affects the equilibrium solution. The FE simulation then predicts a slight decrease in volumetric stress $\sigma_{v h}$ at each radial coordinate for $P_{h} / P_{o}=1.6$ (Fig. 2), shown at the mid-thickness for different pressures in Fig. 4. For $K_{\tau} / K_{\sigma}=1$ and $P_{h} / P_{o}=1.6, \sigma_{v h} / \sigma_{v o}=\tau_{w h} / \tau_{w o} \approx 0.94$ leads to an increase in inner radius $a_{h} / a_{o}=\left(\tau_{w o} / \tau_{w h}\right)^{1 / 3} \approx 1.02$, much lower than for $K_{\tau} / K_{\sigma}=0$, and consistent with analytical solutions. The nearly preserved inner radius (circumferential stretch) and increase in thickness (radial stretch), with an associated increase in volume (Jacobian), closely follow the mechano-adaptive limits $a_{h} / a_{o} \rightarrow \epsilon_{h}^{1 / 3}$ and $h_{h} / h_{o} \rightarrow \gamma_{h} \epsilon_{h}^{1 / 3}$. Yet, a slight, non-ideal over-thickening $(\sim 10 \%$, due to the presence of elastin, which does not turnover and prevents perfect adaptations) was consistent with the slight decrease in $\sigma_{v h}$ due to a decrease in circumferential $(\sim 10 \%)$ rather than axial (which remained nearly constant) stress. Importantly, the pressure-driven mechanobiologically equilibrated (analytical and FE) solutions followed this trend up to $P_{h} / P_{o}=2$, hence suggesting a mechanobiologically stable (bounded) quasi-static evolution within physiological limits [8].

Similar quasi-uniform FE simulations were performed for prescribed changes in flow rate $Q_{h}>Q_{o}$ (Figure 5) and axial stretch $\lambda_{h}>\lambda_{o}$ (Figure 6) with or without shear stress effects on G\&R. Like the pressure-driven simulations addressed above, values computed at the mid-thickness agreed well with the analytical calculations. In particular, an artery does not respond to increases in flow if $K_{\tau}=0$, whereas it mechanoadapts for $K_{\tau}=K_{\sigma}$ with, unlike the previous pressure-driven cases, an associated increase in volumetric stress consistent with a computed increase in flow-induced wall shear stress $\tau_{w h} / \tau_{w o}=\epsilon_{h} /\left(a_{h} / a_{o}\right)^{3} \approx 1.05$ for $\epsilon_{h}=1.6$ (Figure 5). Consideration of shear stress effects to achieve mechanoadaptation was even more critical for isolated changes in axial stretch (i.e., with $\epsilon_{h}=\gamma_{h}=1$ ). Indeed, Figure 6 shows that the artery increased its length $l_{h} / l_{o}=\lambda_{z h}>1$ while nearly preserving inner radius $\left(\lambda_{\theta h} \approx 1\right)$ and thickness $\left(\lambda_{r h} \approx 1\right)$ if $K_{\tau}=K_{\sigma}$, hence $J_{h}>1$ (i.e., by net mass production), indicative of a mechanical adaptation in terms of intramural volumetric and wall shear stresses. Note, however, that axial stress necessarily increases consistent with the axially stretched (elastic) elastin, with circumferential stress regulated and nearly maintained consistent with the slight change in inner radius and thickness with constant blood pressure. In contrast, inner radius $\left(\lambda_{\theta h}<1\right)$ and thickness $\left(\lambda_{r h}<1\right)$ decreased for $K_{\tau}=0$, which even led to respective decrements in volume $J_{h}<1$ (i.e., by net mass removal) for $l_{h} / l_{o}=\lambda_{z h}>1.2$, indicative of a mechanical maladaptation by flow shear stress dysregulation along with excessive increments and decrements of axial and circumferential stresses, respectively, even if the volumetric stress is preserved. Again, the elastically stretched elastin, and its associated increase in axial stress at the mixture level, plays a central role in this lack of adaptation by G\&R.

Although it was possible to compute these quasi-uniform solutions in single load steps during Stage II in Box 3, we nevertheless re-computed them with 10 incremental load steps (plus an initial one for Stage I; symbols in Figs. 4 to 6) to compare quasi-static evolutions with the analytical formulation. Table 2 shows associated quadratic rates of convergence for residual force and energy during global Newton-Raphson iterations, without line searches, and total simulation times (on a single CPU processor Intel ${ }^{\circledR}$ Xeon ${ }^{\circledR}$ E5 at $3 \mathrm{GHz}$ in a Workstation Dell Precision 5810 with $32 \mathrm{~GB}$ RAM). Note that the computational efficiency of this rate-independent G\&R formulation is comparable to that obtained for hyperelastic computations, for which we used the same mesh, boundary conditions, applied loads, and incremental steps along with Eqs. (49) and (50), and, hence, is expected to be comparable as well to that obtained by efficient rate-dependent G\&R formulations (e.g., in 2D FE analyses [37]). Lastly, because mechanobiologically equilibrated G\&R is not constrained isochorically (recall Remark 1), no special formulations were needed to prevent volumetric locking during these computations, a numerical issue characteristic of nearly incompressible elastic or 
distortional elastoplastic responses [24, 38], but also transient elastic responses during G\&R [36, 39]. In this respect, all solution variables changed gradually between adjacent elements and agreed well with analytical results, including global geometric outputs (e.g., inner radius and thickness).

\subsection{Non-uniform equilibrated GER response (Stage II) to localized degradation of elastin}

Next, consider non-uniform deformations of an arterial segment. Since the idealized formula $\tau_{w}=$ $4 \mu Q / \pi a^{3}$ is only valid for fully developed steady flows in a long circular segment, we first considered G\&R driven by intramural stresses only, for which $K_{\sigma} \neq 0$ and $K_{\tau}=0$, such that volumetric stresses were preserved locally, recall Eq. (35). As an example, a G\&R response that can be modeled with $K_{\tau}=0$ in $\Upsilon$ is the enlargement of fusiform aneurysms, where the medial layer appears to be severely damaged (hence no shear stress regulation of smooth muscle tone) and enlargement likely occurs primarily via turnover of remnant collagen in the adventitia [12, 35, 40-43].

Since medial damage implies degradation of elastin laminae, consider the mechanobiologically equilibrated G\&R analysis in Figure 7 performed for a uniform, progressive degradation of elastin, either with decreasing $c_{h}^{e}<c_{o}^{e}$ or an increasing damage parameter $\varphi_{h}^{e}=1-c_{h}^{e} / c_{o}^{e} \in[0,1]$, while preserving inner pressure $P_{h}=P_{o}$, cf. [8]. Due to the close agreement between the uniform analytical and (quasi-)uniform FE simulations shown in Fig. 7 , for both $K_{\tau} / K_{\sigma}=0$ and a slight $K_{\tau} / K_{\sigma}=0.1$, these results provide important guidance for the interpretation of more complex FE simulations. In this case, uniformly degraded elastin, without shear stress effects on G\&R, can eventually lead to an unbounded G\&R response, specifically for $\varphi_{h}^{e} \rightarrow 0.725$ (Fig. $7, K_{\tau} / K_{\sigma}=0$ ), which suggests that locally degraded elastin coupled with endothelial dysfunction could associate with local aneurysmal expansions that may develop quasistatically but remain bounded because constraints on lateral displacements prevent a local asymptotic enlargement. To test this hypothesis within our rate-independent G\&R framework, consider the same initial cylindrical geometry (hence, the same homeostatic state after Stage I), although discretized with 60 elements along the axial direction to enable finer non-uniformities within the central region (see meshes (a) in Figure 8, computed after Stage I). Subsequently, we locally increase $\varphi_{h}^{e}$ at selected regions to drive quasi-static G\&R FE simulations. This way, we model effects of elastin degradation on fully resolved $\mathrm{G} \& \mathrm{R}$, not the underlying cause of this degradation, for which one would need to couple a damage model with the present rate-independent formulation.

With preserved inner pressure $P_{h}=P_{o}$, consider two different spatial distributions for $\varphi_{h}^{e}$ in terms of cylindrical coordinates $r_{o} \in\left[a_{o}, a_{o}+h_{o}\right], \theta_{o} \in[0,2 \pi]$, and $z_{o} \in\left[z_{o m i n}, z_{o m a x}\right]$ in the reference homeostatic configuration $\kappa_{o}$, where $z_{o \text { min }}$ and $z_{o \max }=z_{o \text { min }}+l_{o}$ are minimum and maximum axial coordinates. Consider first an axisymmetric degradation localized at $z_{\text {om }}=\left(z_{o \text { max }}+z_{o m i n}\right) / 2$ that diminishes gradually with $\left|z_{o}-z_{o m}\right|$, namely

$$
\varphi_{h}^{e}\left(r_{o}, \theta_{o}, z_{o}\right)=\varphi_{h \mathrm{~m}}^{e} \exp \left(-\left|\frac{z_{o}-z_{o \mathrm{~m}}}{z_{o \mathrm{~d}}}\right|^{\nu_{z}}\right)
$$

with $\nu_{z}>0$ and $z_{\text {od }}>0$ respective axial exponential decay and deviation parameters. Moreover, $\varphi_{h \mathrm{~m}}^{e}=$ $\varphi_{h}^{e}\left(r_{o}, \theta_{o}, z_{o m}\right)$ describes the maximum local degradation at a given "load" step, and $\varphi_{h}^{e}\left(r_{o}, \theta_{o}, z_{o m i n}\right)=$ $\varphi_{h}^{e}\left(r_{o}, \theta_{o}, z_{\text {omax }}\right) \rightarrow 0$ at both ends. Also, $\varphi_{o}^{e}\left(r_{o}, \theta_{o}, z_{o}\right)=0=\varphi_{o m}^{e}$ throughout the arterial wall at the original homeostatic state. Then, for constant blood pressure, the FE simulation can be advanced in a quasi-static (time-independent) manner by increasing a single scalar $\varphi_{h \mathrm{~m}}^{e} \in[0,1]$ over subsequent steps.

In a first attempt to reproduce this local enlargement of the artery, we prescribed $K_{\tau}=0$ throughout. Because axial displacements are fixed at the boundary ends, and a circumferential expansion with an overall axial retraction was observed at the central region of the artery, local axial stretching is expected next to the ends. Recalling the G\&R response against increases in axial stretch with $K_{\tau}=0$ (Figure 6), one would expect a constriction of the artery close to the ends of the model. Albeit not shown, this abnormal response was verified numerically with our FE model, which suggested the need for a shear stress effect on G\&R, with associated inner radius regulation, in the non-damaged areas. Hence, as a first approximation (in the absence of a fluid-solid interaction solution), we simulated this effect by letting 
$K_{\tau}>0$ (along with $\tau_{w}=4 \mu Q / \pi a^{3}$, recall Eq. (79)) next to the lateral ends, which yet remain cylindrical, but $K_{\tau}=0$ in a central damaged area where the aneurysm develops. To do so, we considered an axial distribution for $K_{\tau} / K_{\sigma}$ similar to that for elastin degradation in Eq. (53), namely

$$
\frac{K_{\tau}}{K_{\sigma}}\left(z_{o}\right)=\frac{K_{\tau}}{K_{\sigma}}\left(z_{\text {omin }}\right)\left(1-\exp \left(-\left|\frac{z_{o}-z_{o \mathrm{~m}}}{z_{\text {od }}}\right|^{\nu_{z}}\right)\right)
$$

Thus, $K_{\tau} / K_{\sigma}\left(z_{\text {omin }}\right)=K_{\tau} / K_{\sigma}\left(z_{\text {omax }}\right)>0$ is maximum at both ends and $K_{\tau} / K_{\sigma}\left(z_{o m}\right)=0$ at the central cross-section. Meshes (b) in Figure 8 show the deformed configuration and associated contour plots of the volume ratio (left) and volumetric stress (right) for $\varphi_{h \mathrm{~m}}^{e}=0.65, z_{o \mathrm{~d}}=l_{o} / 4, \nu_{z}=2$, and $K_{\tau} / K_{\sigma}\left(z_{o m i n}\right)=$ 0.35 in Eqs. (53) and (54), which forms an axisymmetric fusiform aneurysm. Importantly, while the uniform formulation, with $\varphi_{h}^{e}=0.65$ and $K_{\tau}=0$, predicts a nearly isotropic expansion within a given cross-section, with $\lambda_{\theta h}=a_{h} / a_{o}=3.97$ and $\lambda_{r h}=h_{h} / h_{o}=4.17$, and $\lambda_{z h}=l_{h} / l_{o}=1$ prescribed (cf. Figure 7 ), the non-uniform 3D FE simulation predicts a different expansion at the central cross-section $z_{o m}$, with $\lambda_{\theta h}=2.50 \simeq a_{h} / a_{o}$ and $\lambda_{r h}=1.44 \simeq h_{h} / h_{o}$ at the mid-thickness, but $\lambda_{z h}=1.50 \neq l_{h} / l_{o}$. Furthermore, lateral, radially unexpanded, cross-sections yet cause the aneurysm to grow axially at $z_{o m}$ $\left(\lambda_{z h}=1.50\right.$ vs. 1 for the uniform case) and prevent it from growing asymptotically, reducing growth along circumferential ( $\lambda_{\theta h}=2.50$ vs. 3.97$)$ but especially radial ( $\lambda_{r h}=1.44$ vs. 4.17$)$ directions, that is, reduced dilation but especially thickening. In contrast, mean in-plane mechanobiologically equilibrated stresses at $z_{\mathrm{om}}\left(\sigma_{\theta \theta h}=215 \mathrm{kPa}\right.$ and $\left.\sigma_{z z h}=259 \mathrm{kPa}\right)$ remain close to the uniform predictions $\left(\sigma_{\theta \theta h}=214 \mathrm{kPa}\right.$ and $\left.\sigma_{z h}=260 \mathrm{kPa}\right)$, cf. Figure 7 . Note, too, that a marked axial retraction predicted at the shoulders of the aneurysm contributes to an overall retraction of the central region. Hence, as mentioned above, finite elements close to the boundaries $z_{\text {omin }}$ and $z_{\text {omax }}$ are highly stretched axially (reaching up to $\lambda_{z h}=1.69$ ), which justify the distribution for $K_{\tau} / K_{\sigma}$ in Eq. (54) to prevent radial constriction, to some extent, by wall shear stress regulation (recall the analysis in Figure 6, conducted up to a more moderate $\lambda_{z h}=1.3$ ) at the expense of an increased volumetric stress $\sigma_{v h}$ in those regions (mesh (b)-right in Figure 8) consistent with an increased shear stress for constant cardiac output and reduced lumen. The volumetric stress then rapidly decreases in the transition zone where the inner radius starts to increase, consistent with a lower shear stress combined with some remnant $K_{\tau} / K_{\sigma}>0$, to finally recover the original homeostatic distribution for $\sigma_{v o}$ within the aneurysmal region (compare to mesh (a)-right), consistent with a vanishing shear stress effect for $K_{\tau} / K_{\sigma}=0$.

Indeed, it is instructive to consider a different set of boundary conditions at both ends $z_{\text {omin }}$ and $z_{\text {omax }}$ to analyze different responses of the nearby elements. Hence, to alleviate the constraint on axial displacements as well as the associated axial stretching of elastin and consequent radial constriction of the artery, we repeated the previous simulation while prescribing a constant axial force at the ends, coupled with $K_{\tau}=0$ throughout the arterial segment. Meshes (c) in Figure 8 show the formation of an axisymmetric fusiform aneurysm similar to the previous one (b), though with a pronounced axial recoiling $\left(l_{h}=9.85 \mathrm{~mm}\right.$ vs. $\left.l_{o}=15 \mathrm{~mm}\right)$ that effectively alleviates the local axial stretching $\left(\lambda_{z h} \simeq 1\right)$ near the boundaries. Consistent with the analysis in Figure 6, the inner radius at the boundaries is preserved in this case even without considering shear stress effects on G\&R. Also consistent with $K_{\tau}=0$, and unlike the simulation (b), the distribution of $\sigma_{v h}$ is preserved with respect to the original homeostatic preloaded state (a). Equilibrium values at the apex reach, in this case, $\lambda_{\theta h}=2.41, \lambda_{r h}=1.33, \lambda_{z h}=1.11, \sigma_{\theta \theta h}=221 \mathrm{kPa}$, and $\sigma_{z z h}=253 \mathrm{kPa}$, measured at the mid-thickness. Arguably, neither axial displacements nor force are necessarily fixed in actual aneurysms; hence, although these simpler boundary conditions served to illustrate limiting situations and radically different implications, more realistic boundary conditions are needed to simulate aneurysmal enlargement and associated implications.

Consider now a degradation localized at $z_{o m}$ and $\theta_{o m}=\pi$ that diminishes gradually with $\left|z_{o}-z_{o m}\right|$ 
and $\left|\theta_{o}-\theta_{o m}\right|$, namely

$$
\varphi_{h}^{e}\left(r_{o}, \theta_{o}, z_{o}\right)=\varphi_{h \mathrm{~m}}^{e} \exp \left(-\left|\frac{z_{o}-z_{o \mathrm{~m}}}{z_{o \mathrm{~d}}}\right|^{\nu_{z}}\right) \exp \left(-\left|\frac{\theta_{o}-\theta_{o \mathrm{~m}}}{\theta_{o \mathrm{~d}}}\right|^{\nu_{\theta}}\right)
$$

with $\nu_{\theta}>0$ and $\theta_{o \mathrm{~d}}>0$. Moreover, $\varphi_{h \mathrm{~m}}^{e}=\varphi_{h}^{e}\left(r_{o}, z_{o \mathrm{~m}}, \theta_{o \mathrm{~m}}\right)$ describes maximum local degradation at a given "load" step, $\varphi_{h}^{e}\left(r_{o}, \theta_{o}, z_{o m i n}\right)=\varphi_{h}^{e}\left(r_{o}, \theta_{o}, z_{o m a x}\right) \rightarrow 0$ at both ends, and $\varphi_{h}^{e}\left(r_{o}, 0, z_{o}\right)=$ $\varphi_{h}^{e}\left(r_{o}, 2 \pi, z_{o}\right) \rightarrow 0$. As before, we advance the quasi-static FE simulation, for constant inner pressure, by increasing a single scalar $\varphi_{h \mathrm{~m}}^{e} \in[0,1]$ over subsequent steps. Meshes (d) in Figure 8 show the deformed configuration as well as contour plots for $J_{h}$ and $\sigma_{v h}$, with $\varphi_{h \mathrm{~m}}^{e}=0.65, z_{o \mathrm{~d}}=l_{o} / 3, \theta_{o \mathrm{~d}}=\pi / 3$, and $\nu_{z}=\nu_{\theta}=5$ in Eq. (55). This yields an axially longer but radially asymmetric fusiform aneurysm. Axially non-uniform shear stress effects were included again, with $K_{\tau} / K_{\sigma}\left(z_{o \text { omin }}\right)=0.35, z_{o \mathrm{~d}}=l_{o} / 3$, and $\nu_{z}=5$, in Eq. (54). Importantly, even if the maximum local value for $\varphi_{h \mathrm{~m}}^{e}$ is the same as in simulations (b) and (c), the larger surrounding area of non-damaged material (in axial and circumferential directions) prevents the aneurysm from growing to a larger extent. In this case, both $\lambda_{\theta h}=2.29>1$ and $\lambda_{r h}=1.35>1$, but $\lambda_{z h}=0.65<1$ at the apex $\left(z_{o}=z_{o m}, \theta_{o}=\pi\right)$, where in-plane local stresses $\left(\sigma_{\theta \theta h}=250 \mathrm{kPa}\right.$ and $\left.\sigma_{z z h}=224 \mathrm{kPa}\right)$ yet remained bounded. Indirectly induced growth at the diametrically opposed location $\left(z_{o}=z_{o \mathrm{~m}}, \theta_{o}=0\right)$ was lower, namely $\lambda_{\theta h}=1.17>1$ and $\lambda_{r h}=1.30>1$, but $\lambda_{z h}=0.78<1$, where in-plane local stresses reached the same equilibrium values as at the apex.

Finally, let an asymmetric degradation of elastin as in Eq. (55), with the same distribution parameters $z_{o \mathrm{~d}}=l_{o} / 3, \theta_{\text {od }}=\pi / 3$, and $\nu_{z}=\nu_{\theta}=5$, as well as $K_{\tau} / K_{\sigma}\left(z_{\text {omin }}\right)=0.35$ in Eq. (54) and simulation (d), develop in parallel with an increase in transmural pressure $P_{h}>P_{o}$. Meshes (e) in Figure 8 show deformed configurations and contour maps for $\varphi_{h \mathrm{~m}}^{e}=0.35$ and $P_{h}=1.5 P_{o}$, where both drivers were increased simultaneously during the quasi-static computation. Compared to the previous case (d), where $P_{h} \equiv P_{o}$ remained constant but elastin degraded to a greater extent up to 0.65 , the uniform increase in pressure accentuated thickening $\left(\lambda_{r h}=2.18\right.$ at mid thickness) and the change in volume (by mass exchange, mesh (e)-left), but not dilation $\left(\lambda_{\theta h}=1.87\right)$ or axial retraction $\left(\lambda_{z h}=0.65\right)$ in the most damaged region $\left(z_{o}=z_{o m}, \theta_{o}=\pi\right)$; it also rendered more uniform the deformation within the crosssection, with $\lambda_{r h}=2.10, \lambda_{\theta h}=1.42$, and $\lambda_{z h}=0.71$ at $\theta_{o}=0$. Mechanobiologically equilibrated stresses remained bounded throughout the arterial wall again, reaching mean values $\sigma_{\theta \theta h}=248 \mathrm{kPa}$ and $\sigma_{z z h}=226 \mathrm{kPa}$ at the central cross section.

All FE simulations - even when non-uniform along radial, circumferential, or axial directions and when computed for marked local degradation of elastin (b to d) combined with simultaneous increases in pressure (e) - were computed over 10 incremental global steps during Stage II, with asymptotically quadratic rates of convergence and CPU times $(\sim 10 \mathrm{~min})$ shown in Table 3 . Hence, the implementation is robust and efficient for computing fully resolved G\&R responses, with possible numerical issues encountered only when locally approaching mechanobiological static instabilities or excessively distorting the FE mesh, as expected. Indeed, given the computational efficiency, we considered full arterial segment geometries in all of the analyses, with up to 38400 elements, even though by obvious symmetry considerations partial sectors of the specimen could have been analyzed equivalently. Importantly, a recent FE implementation of the original heredity-integral constrained mixture G\&R formulation reported 30 hours, on average, to complete a simulation for a $2^{\circ}$ cylindrical sector of an axisymmetric fusiform aneurysm meshed with 1200 elements [36]. Thus, the computational time reduces drastically when one employs a rate-independent counterpart of the full formulation, which, with $\sim 30$ times more elements, and running $\sim 150$ times faster, roughly represents a 3-fold enhancement in computational efficiency. Nevertheless, the present formulation is not designed to replace the more general heredity-integral formulation in all cases. For example, the present implementation cannot compute transient G\&R responses to step changes in blood pressure, flow, or axial stretch, or predict mechanobiological dynamic instabilities, for which the full formulation is yet needed $[8,10,36]$. 


\subsection{A parametric study}

In the previous examples, we constrained the collagen fibres to deposit along referential orientations $\mathbf{a}_{\mathrm{N}}^{\xi}=$ const with prestretches $\mathbf{G}_{\mathrm{N}}^{\xi}=$ const. This modeling assumption may be appropriate for abdominal aortic aneurysms (AAAs) [44]. Nonetheless, collagen fiber orientation appears generally orthotropic and widely dispersed at the most dilated sites in AAAs, for example, with a circumferential alignment of collagen fibers observed in some cases [45]. Given the computational efficiency of the present approach, we parametrically test possible in-plane anisotropic effects on the final shape of developed aneurysms by prescribing a gradual re-orientation for the angle $\alpha_{0}$ at which symmetric diagonal fibers are deposited during mechanobiologically equilibrated evolutions.

Figure 9 shows contour maps for circumferential (first and third rows of panels) and axial (second and fourth rows) G\&R responses of six different aneurysms that result from respective axisymmetric or asymmetric losses of elastic fiber integrity (up to $\varphi_{h \mathrm{~m}}^{e}=0.65$ ) combined with either an alignment $\alpha_{0 h}=\alpha_{0 o} \approx 30^{\circ}$ that remains constant (left column of panels; cf. meshes (b) and (d) in Figure 8), increases modestly up to $\alpha_{0 h}=(4 / 3) \alpha_{0 o} \approx 40^{\circ}$ (central column), or reorients dramatically up to $\alpha_{0 h}=$ $(5 / 3) \alpha_{0 o} \approx 50^{\circ}$ (right column). In general, a constant alignment for diagonal collagen fibers causes an overall axial retraction of the central region of the aneurysms that induces an expansion of the nonaneurysmal lateral regions (as a result of the axially fixed ends, cf. also meshes (c) in Figure 8 for fixed axial tractions); the modest reorientation causes no overall lengthening of the central region or at the ends; the severe reorientation, in contrast, causes an overall axial expansion of the central region and resulting retraction of the regions next to the fixed ends (as observed in, for example, [40] using a bilayered membrane model with layer-specific fiber orientations).

Interestingly, the more pronounced the axial growth during the formation of an asymmetric aneurysm, the more tortuous its luminal centerline, as commonly seen with AAAs, which appears to mark the development of axial-growth-induced tortuosity, that is, an axial-growth-induced lengthening of the aneurysm between fixed ends in the presence of axially and circumferentially geometric and material asymmetries (i.e., in the presence of an asymmetric aneurysm itself).

\section{Discussion}

Many have simulated complex 3D biological growth in diverse soft tissues using a finite (kinematic) growth framework $[2,4,5,46-48]$. Advantages of this approach include its computational tractability, enabling implementations in existing finite element solvers. A prior disadvantage, however, has been the lack of consideration of the different material properties and rates of turnover of the different constituents that make up native and tissue engineered soft tissues.

In this paper, we presented a new constrained mixture formulation that enables computational tractability while retaining the biologically important characteristic of individual constituent properties, including rates of production and removal. This efficiency was achieved by using a mechanobiologically equilibrated framework, which avoids the heredity integral basis of a full constrained mixture model while providing precise information on the long-term "relaxed" solution [7]. This approach is also useful when time scales associated with G\&R are shorter than those associated with perturbations in mechanical or chemical stimuli to which the cells respond [10]. Illustrative solutions for idealized murine arteries subjected to perturbations in blood pressure and flow as well as changes in axial loading or degradation of elastin (Figs. 4 to 7) demonstrated near exact correspondence between the current and prior constrained mixture models. Importantly, the present formulation is also easily implemented within available finite element solvers, though for non-symmetric tangent stiffness matrices. Results were presented for a modified open source code, FEBio, but preliminary simulations showed similar results using ADINA. The associated solutions revealed quadratic convergence with computational efficiency comparable to that for a nonlinear hyperelastic solution, though here for the simultaneous solution of mechanical and mechanobiological equilibrium at load steps that capture evolving geometries, compositions, and properties of interest. 
Enlargement of a fusiform aneurysm was simulated easily (Fig. 8), with results similar to previous studies [35, 40, 41, 49], though revealing an important new mechanobiological finding. Not all past simulations of aneurysmal G\&R have included both pressure-induced wall stress and flow-induced shear stress in their "stimulus functions" for mass production, and in some cases uncontrollable G\&R was noted at the non-aneurysmal ends. The present results demonstrate that flow-induced shear stress-mediated matrix turnover contributes to control the biaxial G\&R near the non-aneurysmal ends. This finding is biologically sensible for the endothelial cells should be mechanobiologically responsive, and so too the underlying smooth muscle cells, in these non-aneurysmal end regions. In contrast, endothelial dysfunction could manifest within the enlarged aneurysmal region, where disturbed flows exist and smooth muscle cell drop-out is prevalent. These model predictions during quasi-static mechanobiologically equilibrated evolutions, complemented with others in [36] that show a dynamic stabilization of the expansion of abdominal aortic aneurysms afforded by similar effects of flow-induced shear stresses [8], thus motivate the need for new regional-specific assessments of cell functionality in these lesions.

For simplicity in presenting this new computational G\&R framework, we did not consider specific criteria for elastin degradation (e.g., induced by low wall shear stress in fluid-solid growth computational frameworks [50-52]) or damage (e.g., induced by excessive stretching [49]), which was prescribed herein as the stimulation driver for the quasi-static computations of aneurysmal enlargement. Simulated effects of varying deposition stretches, rates of turnover, and material stiffness of collagen have been previously investigated parametrically and proven to play fundamental roles in the natural history of AAAs [53, 54]. We explored effects of the degree of wall heterogeneity and anisotropy on the resulting G\&R response in terms of varying orientations of the newly incorporated collagen fibers within the aneurysmal wall (Fig. 9). Consistent with previous studies that predicted local axial expansions influenced by marked axial off-loadings and noted a propensity for the aneurysm to buckle [40,54], our results revealed that increasing ratios of axial to circumferential growth emerged for a prescribed marked loss of local elastic fiber integrity (up to 65\%) in combination with a gradual reorientation of diagonal fibers of collagen towards the circumferential direction, which led to increasing out-of-plane deformations of the aortic centerline for the asymmetric aneurysms. Collectively, these results highlight the importance of considering anisotropic G\&R when modeling biological tissues $[55,56]$ and, especially, of quantifying not only circumferential growth responses (i.e., maximum diameter) but also axial growth responses (i.e., overall lengthening), along with their expansion rates, when assessing the potential of aneurysms to rupture $[57,58]$. In this regard, the present approach need not prescribe a predefined directionality for the G\&R or associated deformation tensors; rather, case-specific G\&R responses emerge from the simultaneous solution of mechanical (Eq. (7) 2 ) and mechanobiological (Eq. (6) $)_{1}$ ) equilibrium equations complemented by mechanobiologically-inspired constitutive relations for constituent stresses (Section 2.5) and stimulus functions (Section 2.6) subject to case-specific boundary conditions (see also [56]).

Although full, heredity-based constrained mixture models (e.g., [12, 15, 31, 32]) will continue to be needed to study particular physiological and pathophysiological situations, the present formulation appears to apply in many cases, provided that the loading or structural perturbations remain static or are slow enough relative to the G\&R process. We submit, therefore, that this easily implemented, fast, rate-independent finite element approach to modeling soft tissue G\&R will enable previously impractical parametric studies that can be used to evaluate novel mechanobiological hypotheses, which in turn can better focus cost- and time-intensive experimental studies for a host of soft tissues [11].

There is, of course, further need to understand better the actual mechanical stimuli that the cells sense and respond to, noting that different cells may respond to different magnitudes or even different stimuli. We considered $\sigma_{v o}$ and $\tau_{w o}$ as homeostatic targets, which yielded numerical responses that reflect experimental observations. Much more remains to be discovered, and then modeled, in soft tissue mechanobiology. 
bioRxiv preprint doi: https://doi.org/10.1101/2020.02.27.968768; this version posted May 9, 2020. The copyright holder for this preprint (which was not certified by peer review) is the author/funder. All rights reserved. No reuse allowed without permission.

\section{Acknowledgments}

This work was supported, in part, by grants from the NIH (R01 HL128602, P01 HL134605, U01 HL142518) and DoD (W81 XWH1810518).

\section{Declaration of Interest}

The authors declare no conflict of interest, financial or otherwise. 


\section{References}

[1] S. C. Cowin, Tissue growth and remodeling, Annual Review of Biomedical Engineering 6 (2004) $77-107$.

[2] A. Menzel, E. Kuhl, Frontiers in growth and remodeling, Mechanics research communications 42 (2012) 1-14.

[3] K. Myers, G. A. Ateshian, Interstitial growth and remodeling of biological tissues: tissue composition as state variables, Journal of the mechanical behavior of biomedical materials 29 (2014) 544-556.

[4] A. Goriely, The mathematics and mechanics of biological growth, Vol. 45, Springer, 2017.

[5] D. Ambrosi, M. Ben Amar, C. J. Cyron, A. DeSimone, A. Goriely, J. D. Humphrey, E. Kuhl, Growth and remodelling of living tissues: perspectives, challenges and opportunities, Journal of the Royal Society Interface 16 (157) (2019) 20190233.

[6] J. D. Humphrey, K. R. Rajagopal, A constrained mixture model for growth and remodeling of soft tissues, Mathematical Models and Methods in Applied Sciences 12 (03) (2002) 407-430.

[7] M. Latorre, J. D. Humphrey, A mechanobiologically equilibrated constrained mixture model for growth and remodeling of soft tissues, ZAMM-Journal of Applied Mathematics and Mechanics 98 (2018) 2048-2071.

[8] M. Latorre, J. D. Humphrey, Mechanobiological stability of biological soft tissues, Journal of the Mechanics and Physics of Solids 125 (2019) 298-325.

[9] K. J. A. Davies, Adaptive homeostasis, Molecular aspects of medicine 49 (2016) 1-7.

[10] M. Latorre, J. D. Humphrey, Critical roles of time-scales in soft tissue growth and remodeling, APL Bioengineering 2 (2) (2018) 026108.

[11] M. Latorre, Modeling biological growth and remodeling: contrasting methods, contrasting needs, Current Opinion in Biomedical Engineering 15 (2020) 26-31.

[12] S. Baek, K. R. Rajagopal, J. D. Humphrey, A theoretical model of enlarging intracranial fusiform aneurysms, Journal of biomechanical engineering 128 (1) (2006) 142-149.

[13] J. D. Humphrey, E. R. Dufresne, M. A. Schwartz, Mechanotransduction and extracellular matrix homeostasis, Nature Reviews Molecular Cell Biology 15 (12) (2014) 802-812.

[14] A. Valentín, J. D. Humphrey, G. A. Holzapfel, A finite element-based constrained mixture implementation for arterial growth, remodeling, and adaptation: Theory and numerical verification, International journal for numerical methods in biomedical engineering 29 (8) (2013) 822-849.

[15] M. Latorre, J. D. Humphrey, Modeling mechano-driven and immuno-mediated aortic maladaptation in hypertension, Biomechanics and Modeling in Mechanobiology 17 (5) (2018) 1497-1511.

[16] A. Menzel, A fibre reorientation model for orthotropic multiplicative growth, Biomechanics and modeling in mechanobiology 6 (5) (2007) 303-320.

[17] Y. C. Fung, Stress, strain, growth, and remodeling of living organisms, in: J. Casey, M. J. Crochet (Eds.), Theoretical, Experimental, and Numerical Contributions to the Mechanics of Fluids and Solids, Birkhäuser, Basel, 1995, pp. 469-482.

[18] J. D. Humphrey, Cardiovascular Solid Mechanics: Cells, Tissues and Organs, Springer-Verlag, New York, 2002. 
[19] G. A. Holzapfel, Nonlinear Solid Mechanics. A Continuum Approach for Engineering, John Wiley \& Sons, Chichester, 2000.

[20] M. Epstein, G. A. Maugin, Thermomechanics of volumetric growth in uniform bodies, International Journal of Plasticity 16 (7) (2000) 951-978.

[21] G. Himpel, E. Kuhl, A. Menzel, P. Steinmann, Computational modelling of isotropic multiplicative growth, Computer Modeling in Engineering \& Sciences 8 (2) (2005) 119-134.

[22] K. C. Bennett, R. A. Regueiro, R. I. Borja, Finite strain elastoplasticity considering the Eshelby stress for materials undergoing plastic volume change, International Journal of Plasticity 77 (2016) $214-245$.

[23] J. C. Simó, Algorithms for static and dynamic multiplicative plasticity that preserve the classical return mapping schemes of the infinitesimal theory, Computer Methods in Applied Mechanics and Engineering 99 (1) (1992) 61-112.

[24] M. Á. Sanz, F. J. Montáns, M. Latorre, Computational anisotropic hardening multiplicative elastoplasticity based on the corrector elastic logarithmic strain rate, Computer Methods in Applied Mechanics and Engineering 320 (2017) 82-121.

[25] C. Vignes, P. Papadopoulos, Material growth in thermoelastic continua: theory, algorithmics, and simulation, Computer Methods in Applied Mechanics and Engineering 199 (17-20) (2010) 979-996.

[26] J. C. Simó, R. L. Taylor, Consistent tangent operators for rate-independent elastoplasticity, Computer Methods in Applied Mechanics and Engineering 48 (1) (1985) 101-118.

[27] K. Schwlizerhof, E. Ramm, Displacement dependent pressure loads in nonlinear finite element analyses, Computers \& Structures 18 (6) (1984) 1099-1114.

[28] K.-J. Bathe, Finite element procedures, 2nd Edition, Klaus-Jürgen Bathe, 2014.

[29] M. Latorre, F. J. Montáns, Fully anisotropic finite strain viscoelasticity based on a reverse multiplicative decomposition and logarithmic strains, Computers \& Structures 163 (2016) 56-70.

[30] C. Bellini, J. Ferruzzi, S. Roccabianca, E. S. Di Martino, J. D. Humphrey, A microstructurally motivated model of arterial wall mechanics with mechanobiological implications, Annals of Biomedical Engineering 42 (3) (2014) 488-502.

[31] S. Baek, A. Valentín, J. D. Humphrey, Biochemomechanics of cerebral vasospasm and its resolution: II. constitutive relations and model simulations, Annals of Biomedical Engineering 35 (9) (2007) 1498.

[32] K. S. Miller, R. Khosravi, C. K. Breuer, J. D. Humphrey, A hypothesis-driven parametric study of effects of polymeric scaffold properties on tissue engineered neovessel formation, Acta biomaterialia 11 (2015) 283-294.

[33] S. A. Maas, B. J. Ellis, G. A. Ateshian, J. A. Weiss, Febio: finite elements for biomechanics, Journal of biomechanical engineering 134 (1) (2012) 011005.

[34] S. J. Mousavi, S. Avril, Patient-specific stress analyses in the ascending thoracic aorta using a finite-element implementation of the constrained mixture theory, Biomechanics and modeling in mechanobiology 16 (5) (2017) 1765-1777. 
[35] S. Zeinali-Davarani, A. Sheidaei, S. Baek, A finite element model of stress-mediated vascular adaptation: application to abdominal aortic aneurysms, Computer methods in biomechanics and biomedical engineering 14 (9) (2011) 803-817.

[36] N. Horvat, L. Virag, G. A. Holzapfel, J. Sorić, I. Karšaj, A finite element implementation of a growth and remodeling model for soft biological tissues: Verification and application to abdominal aortic aneurysms, Computer Methods in Applied Mechanics and Engineering 352 (2019) 586-605.

[37] J. D. Laubrie, J. S. Mousavi, S. Avril, A new finite-element shell model for arterial growth and remodeling after stent implantation, International Journal for Numerical Methods in Biomedical Engineering 36 (1) (2020) e3282.

[38] T. Sussman, K.-J. Bathe, A finite element formulation for nonlinear incompressible elastic and inelastic analysis, Computers \& Structures 26 (1-2) (1987) 357-409.

[39] P.-W. Fok, K. Gou, Finite element simulation of intimal thickening in 2D multi-layered arterial cross sections by morphoelasticity, Computer Methods in Applied Mechanics and Engineering 363 (2020) 112860 .

[40] P. N. Watton, N. A. Hill, M. Heil, A mathematical model for the growth of the abdominal aortic aneurysm, Biomechanics and modeling in mechanobiology 3 (2) (2004) 98-113.

[41] C. J. Cyron, J. S. Wilson, J. D. Humphrey, Mechanobiological stability: a new paradigm to understand the enlargement of aneurysms?, Journal of the Royal Society Interface 11 (100) (2014) 20140680 .

[42] W. Lin, M. Iafrati, R. Peattie, L. Dorfmann, Growth and remodeling with application to abdominal aortic aneurysms, Journal of Engineering Mathematics 109 (1) (2018) 113-137.

[43] S. J. Mousavi, S. Farzaneh, S. Avril, Patient-specific predictions of aneurysm growth and remodeling in the ascending thoracic aorta using the homogenized constrained mixture model, Biomechanics and modeling in mechanobiology 18 (6) (2019) 1895-1913.

[44] G. Urabe, K. Hoshina, T. Shimanuki, Y. Nishimori, T. Miyata, J. Deguchi, Structural analysis of adventitial collagen to feature aging and aneurysm formation in human aorta, Journal of vascular surgery 63 (5) (2016) 1341-1350.

[45] T. C. Gasser, S. Gallinetti, X. Xing, C. Forsell, J. Swedenborg, J. Roy, Spatial orientation of collagen fibers in the abdominal aortic aneurysm's wall and its relation to wall mechanics, Acta biomaterialia 8 (8) (2012) 3091-3103.

[46] E. Kuhl, R. Maas, G. Himpel, A. Menzel, Computational modeling of arterial wall growth, Biomechanics and modeling in mechanobiology 6 (5) (2007) 321-331.

[47] M. Ben Amar, A. Goriely, Growth and instability in elastic tissues, Journal of the Mechanics and Physics of Solids 53 (10) (2005) 2284-2319.

[48] D. Ambrosi, A. Guillou, E. S. Di Martino, Stress-modulated remodeling of a non-homogeneous body, Biomechanics and modeling in mechanobiology 7 (1) (2008) 63-76.

[49] J. S. Wilson, S. Baek, J. D. Humphrey, Importance of initial aortic properties on the evolving regional anisotropy, stiffness and wall thickness of human abdominal aortic aneurysms, Journal of The Royal Society Interface 9 (74) (2012) 2047-58. 
[50] A. Sheidaei, S. C. Hunley, S. Zeinali-Davarani, L. G. Raguin, S. Baek, Simulation of abdominal aortic aneurysm growth with updating hemodynamic loads using a realistic geometry, Medical engineering \& physics 33 (1) (2011) 80-88.

[51] P. Aparicio, A. Mandaltsi, J. Boamah, H. Chen, A. Selimovic, M. Bratby, R. Uberoi, Y. Ventikos, P. N. Watton, Modelling the influence of endothelial heterogeneity on the progression of arterial disease: application to abdominal aortic aneurysm evolution, International journal for numerical methods in biomedical engineering 30 (5) (2014) 563-586.

[52] A. Grytsan, P. N. Watton, G. A. Holzapfel, A thick-walled fluid-solid-growth model of abdominal aortic aneurysm evolution: application to a patient-specific geometry, Journal of biomechanical engineering 137 (3) (2015).

[53] G. Martufi, T. C. Gasser, Turnover of fibrillar collagen in soft biological tissue with application to the expansion of abdominal aortic aneurysms, Journal of the Royal Society Interface 9 (77) (2012) $3366-3377$.

[54] J. S. Wilson, S. Baek, J. D. Humphrey, Parametric study of effects of collagen turnover on the natural history of abdominal aortic aneurysms, Proceedings of the Royal Society A: Mathematical, Physical and Engineering Sciences 469 (2150) (2013) 20120556.

[55] A. Grytsan, T. S. E. Eriksson, P. N. Watton, T. C. Gasser, Growth description for vessel wall adaptation: A thick-walled mixture model of abdominal aortic aneurysm evolution, Materials 10 (9) (2017) 994.

[56] F. A. Braeu, R. C. Aydin, C. J. Cyron, Anisotropic stiffness and tensional homeostasis induce a natural anisotropy of volumetric growth and remodeling in soft biological tissues, Biomechanics and Modeling in Mechanobiology 18 (2) (2019) 327-345.

[57] M. F. Fillinger, J. Racusin, R. K. Baker, J. L. Cronenwett, A. Teutelink, M. L. Schermerhorn, R. M. Zwolak, R. J. Powell, D. B. Walsh, E. M. Rzucidlo, Anatomic characteristics of ruptured abdominal aortic aneurysm on conventional ct scans: implications for rupture risk, Journal of vascular surgery 39 (6) (2004) 1243-1252.

[58] S. Pappu, A. Dardik, H. Tagare, R. J. Gusberg, Beyond fusiform and saccular: a novel quantitative tortuosity index may help classify aneurysm shape and predict aneurysm rupture potential, Annals of vascular surgery 22 (1) (2008) 88-97.

[59] I. Hariton, G. deBotton, T. C. Gasser, G. A. Holzapfel, Stress-driven collagen fiber remodeling in arterial walls, Biomechanics and modeling in mechanobiology 6 (3) (2007) 163-175.

[60] C. A. Figueroa, S. Baek, C. A. Taylor, J. D. Humphrey, A computational framework for fluidsolid-growth modeling in cardiovascular simulations, Computer Methods in Applied Mechanics and Engineering 198 (45) (2009) 3583-3602.

[61] R. W. Ogden, Non-linear elastic deformations, Dover Publications, Mineola, New York, 1997.

[62] M. Latorre, F. J. Montáns, Stress and strain mapping tensors and general work-conjugacy in large strain continuum mechanics, Applied Mathematical Modelling 40 (5) (2016) 3938-3950. 


\section{Appendix A. Deformation gradients during general G\&R responses}

Within a general constrained mixture theory, the deformation gradient for a constituent $\alpha$ deposited at time $\tau$ within extant matrix, and that survives at current G\&R time $s \geq \tau$, reads [12]

$$
\mathbf{F}_{\mathrm{n}(\tau)}^{\alpha}(s)=\mathbf{F}(s) \mathbf{F}^{-1}(\tau) \mathbf{G}^{\alpha}(\tau)
$$

which maps line elements (fibers) from an evolving natural configuration $\kappa_{\mathrm{n}}^{\alpha}(\tau)$ to the current configuration of the mixture $\kappa(s)$, with $\mathbf{G}^{\alpha}(\tau)=\left(\mathbf{G}^{\alpha}(\tau)\right)^{\mathrm{T}}$, and $\operatorname{det} \mathbf{G}^{\alpha}(\tau)=1$. At deposition time $\tau$,

$$
\mathbf{F}_{\mathrm{n}(\tau)}^{\alpha}(\tau)=\mathbf{F}(\tau) \mathbf{F}^{-1}(\tau) \mathbf{G}^{\alpha}(\tau)=\mathbf{G}^{\alpha}(\tau)
$$

thus the natural configuration $\kappa_{\mathrm{n}}^{\alpha}(\tau)$ evolves with the current configuration of the soft tissue at $\tau$. It is for this reason that newly produced collagen families are often assumed to be incorporated within the wall, at a homeostatic stretch, along unit vectors relative to spatial directions (i.e., eigenvectors) of the Cauchy stress tensor in the loaded configuration at time of deposition $[12,14,59]$. Consequently, $\kappa_{\mathrm{n}}^{\alpha}(\tau)$ is subject to possible rigid-body motions superimposed on the current configuration at $\tau$. In other words, the magnitude of $\mathbf{G}^{\alpha}$ (e.g., its eigenvalues) might be assumed to remain constant over time, but not so its spatial orientation (i.e., its eigenvectors).

To develop formulations insensitive to rigid body motions, it is often convenient to define equivalent variables in a referential (Lagrangian) description. Hence, upon considering the right polar decomposition of the deformation gradient

$$
\mathbf{F}(\tau)=\mathbf{R}(\tau) \mathbf{U}(\tau)
$$

and, because of the inherent link between $\kappa_{\mathrm{n}}^{\alpha}(\tau)$ and the corresponding current configuration of the mixture $\kappa(\tau)$, we can define a (yet symmetric, volume-preserving) prestretch tensor rotated by $\mathbf{R}^{\mathrm{T}}(\tau)$ as

$$
\mathbf{G}_{\mathrm{N}}^{\alpha}(\tau):=\mathbf{R}^{\mathrm{T}}(\tau) \mathbf{G}^{\alpha}(\tau) \mathbf{R}(\tau)
$$

which is determined relative to an evolving natural configuration $\kappa_{\mathrm{N}}^{\alpha}(\tau)$ that is rotated with respect to $\kappa_{\mathrm{n}}^{\alpha}(\tau)$ but remains unrotated with respect to the reference configuration of the mixture $\kappa(0)=\kappa_{o}$. This motivates the introduction of a two-point linear transformation defined at the constituent level as

$$
\mathbf{F}_{\mathrm{G}}^{\alpha}(\tau):=\mathbf{G}^{\alpha}(\tau) \mathbf{R}(\tau)=\mathbf{R}(\tau) \mathbf{G}_{\mathrm{N}}^{\alpha}(\tau)
$$

which, importantly, allows one to interpret (the original) $\mathbf{G}^{\alpha}$ and (the herein introduced) $\mathbf{G}_{\mathrm{N}}^{\alpha}$ as respective left and right prestretch tensors defined with respect to the Lagrangian-type natural configuration $\kappa_{\mathrm{N}}^{\alpha}$. Therefore, we can also define (cf. Eq. (56))

$$
\mathbf{F}_{\mathrm{N}(\tau)}^{\alpha}(s):=\mathbf{F}_{\mathrm{n}(\tau)}^{\alpha}(s) \mathbf{R}(\tau)=\mathbf{F}(s) \mathbf{F}^{-1}(\tau) \mathbf{F}_{\mathrm{G}}^{\alpha}(\tau)
$$

which maps line elements (fibers) from $\kappa_{\mathrm{N}}^{\alpha}(\tau)$ to the current configuration of the mixture $\kappa(s)$. Hence, at deposition time $\tau$ (cf. Eq. (57))

$$
\mathbf{F}_{\mathrm{N}(\tau)}^{\alpha}(\tau)=\mathbf{F}(\tau) \mathbf{F}^{-1}(\tau) \mathbf{F}_{\mathrm{G}}^{\alpha}(\tau)=\mathbf{F}_{\mathrm{G}}^{\alpha}(\tau)
$$

In this way, one can conveniently remove possible sources of non-objectivity by considering the rotated (right) deposition stretch tensor $\mathbf{G}_{\mathrm{N}}^{\alpha}$. Here, we posit, for example, that both its magnitude and orientation remain constant

$$
\mathbf{G}_{\mathrm{N}}^{\alpha}(\tau)=\mathbf{G}_{\mathrm{N}}^{\alpha}=\mathbf{c o n s t} \quad \forall \tau \Longrightarrow \mathbf{G}^{\alpha}(\tau)=\mathbf{R}(\tau) \mathbf{G}_{\mathrm{N}}^{\alpha} \mathbf{R}^{\mathrm{T}}(\tau)
$$

In cases that rotations are absent, as, for example, for axisymmetrically loaded arteries treated as thinwalled [7, 8] or thick-walled [14] cylinders, this assumption yields $\mathbf{G}^{\alpha}(\tau)=\mathbf{G}_{N}^{\alpha}=\mathbf{c o n s t} \forall \tau$, as assumed therein. 
For mechanobiologically equilibrated states, when $\mathbf{F}(\tau)=\mathbf{F}(s)=: \mathbf{F}_{h}$, we have, in general

$$
\mathbf{F}_{\mathrm{n} h}^{\alpha}=\mathbf{F}_{h} \mathbf{F}_{h}^{-1} \mathbf{G}^{\alpha}=\mathbf{G}^{\alpha}
$$

and

$$
\mathbf{F}_{\mathrm{N} h}^{\alpha}=\mathbf{F}_{h} \mathbf{F}_{h}^{-1} \mathbf{F}_{\mathrm{G} h}^{\alpha}=\mathbf{F}_{\mathrm{G} h}^{\alpha}=\mathbf{R}_{h} \mathbf{G}_{\mathrm{N}}^{\alpha} .
$$

The latter decomposition, expressed in terms of $\mathbf{R}_{h} \neq$ const and $\mathbf{G}_{\mathrm{N}}^{\alpha}=$ const, proves useful when defining mechanobiologically equilibrated (rotated) Cauchy stresses subject to objective requirements. In this regard, note that an arbitrary rigid-body rotation $\mathbf{Q} \in \mathrm{orth}^{+}$superimposed on $\mathbf{F}_{\mathrm{N} h}^{\alpha}$ yields

$$
\mathbf{Q F}_{\mathrm{N} h}^{\alpha}=\mathbf{Q R}_{h} \mathbf{G}_{\mathrm{N}}^{\alpha}=\mathbf{R}_{h}^{*} \mathbf{G}_{\mathrm{N}}^{\alpha} \equiv \mathbf{F}_{\mathrm{N} h}^{\alpha *}
$$

with the superimposed rotation absorbed by $\mathbf{R}_{h}^{*}=\mathbf{Q} \mathbf{R}_{h}$, and $\mathbf{G}_{\mathrm{N}}^{\alpha}$ remaining unaltered (i.e., constant, defined in $\kappa_{\mathrm{N}}^{\alpha}$ ). In contrast, because $\kappa_{\mathrm{n}}^{\alpha}$ evolves with the current configuration of the mixture, an arbitrary rotation superimposed on $\mathbf{F}_{\mathrm{n} h}^{\alpha}$ implies a rotation on $\kappa_{\mathrm{n}}^{\alpha}$ and, hence, enforces a rotation of $\mathbf{G}^{\alpha}$ by means of

$$
\mathbf{Q F}_{\left(\mathrm{n}^{*}\right) h}^{\alpha}=\mathbf{Q F}_{\mathrm{n} h}^{\alpha} \mathbf{Q}^{\mathrm{T}}=\mathbf{Q G}^{\alpha} \mathbf{Q}^{\mathrm{T}}=\mathbf{G}^{\alpha *} \equiv \mathbf{F}_{\left(\mathrm{n}^{*}\right) h}^{\alpha *}
$$

with $\mathbf{G}^{\alpha *}=\mathbf{Q G}^{\alpha} \mathbf{Q}^{\mathrm{T}}$ defined in a rotated natural configuration $\kappa_{\mathrm{n}^{*}}^{\alpha}$, and $\mathbf{Q}^{\mathrm{T}} \equiv \mathbf{Q}^{-1}$ rotating line elements from $\kappa_{\mathrm{n}^{*}}^{\alpha}$ to $\kappa_{\mathrm{n}}^{\alpha}$ accordingly. Again, note that $\mathbf{F}_{\mathrm{n} h}^{\alpha}=\mathbf{G}^{\alpha}=\mathbf{c o n s t}$ for cylindrical geometries $[7,8]$.

\section{Appendix B. Stresses during transient hyperelastic responses}

The energy $W_{\mathrm{R}}\left(\mathbf{C}_{h}\right)$ in Eq. (23) gives that stored by the mixture as a consequence of its pre-stretched in vivo state. To determine associated pre-stresses by differentiation of $W_{\mathrm{R}}$ under the assumption that the bulk modulus of the tissue far exceeds its shear modulus (i.e., volume may change with growth but not loading over short periods), one can consider arbitrary isochoric (elastic) deformations superimposed to the equilibrated current configuration of the mixture $\kappa_{h}$. Particularization of these expressions to $\mathbf{F} \equiv \mathbf{F}_{h}$ provides mechanobiologically equilibrated stresses.

Consider the combined gradient $\mathbf{F}=\overline{\mathbf{F}} \mathbf{F}_{h}$, with $\mathbf{F}_{h}$ fixed and $\overline{\mathbf{F}}$ superimposed. From $\mathbf{F}^{\zeta}=\overline{\mathbf{F}} \mathbf{F}_{h}^{\zeta}=$ $\mathbf{F F}_{h}^{-1} \mathbf{F}_{h}^{\zeta}=\mathbf{F G}^{\zeta}, \mathbf{C}^{\zeta}=\mathbf{F}^{\zeta \mathrm{T}} \mathbf{F}^{\zeta}$ yields, with $\mathbf{C}=\mathbf{F}^{\mathrm{T}} \mathbf{F}$,

$$
\mathbf{C}^{\zeta}=\mathbf{G}^{\zeta} \mathbf{C G}^{\zeta}=\mathbf{G}^{\zeta} \odot \mathbf{G}^{\zeta}: \mathbf{C}=\mathbf{C}^{\zeta}(\mathbf{C}) .
$$

Similarly, from $\mathbf{F}_{\mathrm{N}}^{\xi}=\overline{\mathbf{F}} \mathbf{F}_{\mathrm{N} h}^{\xi}=\mathbf{F F}_{h}^{-1} \mathbf{F}_{\mathrm{N} h}^{\xi}=\mathbf{F} \mathbf{U}_{h}^{-1} \mathbf{G}_{\mathrm{N}}^{\xi}, \mathbf{C}_{\mathrm{N}}^{\xi}=\mathbf{F}_{\mathrm{N}}^{\xi \mathrm{T}} \mathbf{F}_{\mathrm{N}}^{\xi}$ yields

$$
\mathbf{C}_{\mathrm{N}}^{\xi}=\mathbf{G}_{\mathrm{N}}^{\xi} \mathbf{U}_{h}^{-1} \mathbf{C} \mathbf{U}_{h}^{-1} \mathbf{G}_{\mathrm{N}}^{\xi}=\mathbf{G}_{\mathrm{N}}^{\xi} \mathbf{U}_{h}^{-1} \odot \mathbf{G}_{\mathrm{N}}^{\xi} \mathbf{U}_{h}^{-1}: \mathbf{C}=\mathbf{C}_{\mathrm{N}}^{\xi}(\mathbf{C})
$$

Since $\bar{J}=\operatorname{det} \overline{\mathbf{F}}=1$, then $J=\operatorname{det} \mathbf{F}=\bar{J} J_{h}=J_{h}$, and the strain energy for the mixture, particularized to a homeostatic state in Eq. (23), generalizes to

$$
W_{\mathrm{R}}(\mathbf{C})=\sum_{\zeta=1}^{N^{\zeta}} \phi_{o}^{\zeta} \hat{W}^{\zeta}\left(\mathbf{C}^{\zeta}\right)+\sum_{\xi=1}^{N^{\xi}} \phi_{\mathrm{R} h}^{\xi} \hat{W}^{\xi}\left(\mathbf{C}_{\mathrm{N}}^{\xi}\right)
$$

with $\phi_{\mathrm{R} h}^{\xi}=\phi_{\mathrm{R}}^{\xi}\left(J_{h}\right)=J_{h} \phi^{\xi}\left(J_{h}\right)=J_{h} \phi_{h}^{\xi}$ remaining constant.

The stress power per unit reference volume in $\kappa_{O}$ is, for this intermittent hyperelastic response, $\mathcal{P}=\mathbf{S}: \frac{1}{2} \dot{\mathbf{C}}=\dot{W}_{\mathrm{R}}(\mathbf{C})=2 \partial W_{\mathrm{R}}(\mathbf{C}) / \partial \mathbf{C}: \frac{1}{2} \dot{\mathbf{C}}$, with $\mathbf{S}$ the second Piola-Kirchhoff stress for the mixture, 
which yields

$$
\mathbf{S}: \frac{1}{2} \dot{\mathbf{C}}=\left(\sum_{\zeta=1}^{N^{\zeta}} 2 \phi_{o}^{\zeta} \frac{\partial \hat{W}^{\zeta}\left(\mathbf{C}^{\zeta}\right)}{\partial \mathbf{C} \zeta}: \frac{\partial \mathbf{C}^{\zeta}(\mathbf{C})}{\partial \mathbf{C}}+\sum_{\xi=1}^{N^{\xi}} 2 \phi_{\mathrm{R} h}^{\xi} \frac{\partial \hat{W}^{\xi}\left(\mathbf{C}_{\mathrm{N}}^{\xi}\right)}{\partial \mathbf{C}_{\mathrm{N}}^{\xi}}: \frac{\partial \mathbf{C}_{\mathrm{N}}^{\xi}(\mathbf{C})}{\partial \mathbf{C}}-J_{h} p \mathbf{C}^{-1}\right): \frac{1}{2} \dot{\mathbf{C}}
$$

where we accounted for dependencies in Eqs. (68) and (69) and added the last term consistent with the kinematic constraint $\dot{J}=0=J_{h} \mathbf{C}^{-1}: \frac{1}{2} \dot{\mathbf{C}}$, with $p$ the associated Lagrange multiplier. Then, $\forall \dot{\mathbf{C}}$ subject to $\dot{J}=0$, we obtain

$$
\mathbf{S}=\sum_{\zeta=1}^{N^{\zeta}} \phi_{o}^{\zeta} \mathbf{G}^{\zeta} \hat{\mathbf{S}}^{\zeta}\left(\mathbf{C}^{\zeta}\right) \mathbf{G}^{\zeta}+\sum_{\xi=1}^{N^{\xi}} \phi_{\mathrm{R} h}^{\xi} \mathbf{U}_{h}^{-1} \mathbf{G}_{\mathrm{N}}^{\xi} \hat{\mathbf{S}}_{\mathrm{N}}^{\xi}\left(\mathbf{C}_{\mathrm{N}}^{\xi}\right) \mathbf{G}_{\mathrm{N}}^{\xi} \mathbf{U}_{h}^{-1}-J_{h} p \mathbf{C}^{-1}
$$

where we defined second Piola-Kirchhoff stresses at the constituent level for both type of constituents

$$
\hat{\mathbf{S}}^{\zeta}\left(\mathbf{C}^{\zeta}\right)=2 \frac{\partial \hat{W}^{\zeta}\left(\mathbf{C}^{\zeta}\right)}{\partial \mathbf{C}^{\zeta}}, \quad \hat{\mathbf{S}}_{\mathrm{N}}^{\xi}\left(\mathbf{C}_{\mathrm{N}}^{\xi}\right)=2 \frac{\partial \hat{W}^{\xi}\left(\mathbf{C}_{\mathrm{N}}^{\xi}\right)}{\partial \mathbf{C}_{\mathrm{N}}^{\xi}} .
$$

Note that Eqs. (70) and (71), obtained for hyperelastic (isochoric) responses with respect to an evolved, grown and remodeled, state $\kappa_{h}$, constitute a generalization of both the mass averaged rule-of-mixture strain energy and stresses in Ref. [30], obtained for hyperelastic (isochoric) responses with respect to an original homeostatic state $\kappa_{o}$, for which $W_{\mathrm{R}} \equiv W, \phi_{\mathrm{R} h}^{\xi} \equiv \phi_{o}^{\xi}, J_{h} \equiv J_{o}=1$, and $\mathbf{U}_{h} \equiv \mathbf{U}_{o}=\mathbf{I}$, namely

$$
\left.W(\mathbf{C})\right|_{o}=\sum_{\zeta=1}^{N^{\zeta}} \phi_{o}^{\zeta} \hat{W}^{\zeta}\left(\mathbf{C}^{\zeta}\right)+\sum_{\xi=1}^{N^{\xi}} \phi_{o}^{\xi} \hat{W}^{\xi}\left(\mathbf{C}_{\mathrm{N}}^{\xi}\right)
$$

and

$$
\left.\mathbf{S}\right|_{o}=\sum_{\zeta=1}^{N^{\zeta}} \phi_{o}^{\zeta} \mathbf{G}^{\zeta} \hat{\mathbf{S}}^{\zeta}\left(\mathbf{C}^{\zeta}\right) \mathbf{G}^{\zeta}+\sum_{\xi=1}^{N^{\xi}} \phi_{o}^{\xi} \mathbf{G}_{\mathrm{N}}^{\xi} \hat{\mathbf{S}}_{\mathrm{N}}^{\xi}\left(\mathbf{C}_{\mathrm{N}}^{\xi}\right) \mathbf{G}_{\mathrm{N}}^{\xi}-p \mathbf{C}^{-1}
$$

\section{Appendix C. A stimulus function for arterial G\&R}

In previous works, we have analyzed G\&R of prototypical cylindrical arteries with stimulus functions for mass production

$$
\Upsilon_{h}^{\xi}-1=K_{\sigma}^{\xi} \Delta \sigma_{h}-K_{\tau}^{\xi} \Delta \tau_{w h}
$$

where

$$
\Delta \sigma_{h}=\frac{\tilde{\sigma}_{h}-\tilde{\sigma}_{o}}{\tilde{\sigma}_{o}} \quad \text { and } \quad \Delta \tau_{w h}=\frac{\tau_{w h}-\tau_{w o}}{\tau_{w o}}
$$

with $\tilde{\sigma}=\sigma_{I}=\operatorname{tr} \boldsymbol{\sigma}$ the first principal invariant of the mean wall stress and $\tau_{w}=4 \mu Q / \pi a^{3}$ a measure of the wall shear stress over the endothelium for a fully developed Newtonian flow through a long cylindrical sector, with $\mu$ the viscosity of blood, $Q$ the volumetric flow rate, and $a$ the current luminal radius. Note that, while we can still employ $\tilde{\sigma}=\sigma_{I}=\operatorname{tr} \boldsymbol{\sigma}$ in the present general framework, with $\boldsymbol{\sigma}$ now defined pointwise in Eq. (32), we cannot compute local values for $\tau_{w}$ for complex geometries, for which one would need to incorporate computations for the blood flow to account for fluid-solid interactions, namely perform fluid-solid-growth cardiovascular simulations [60] particularized in the present case to quasi-steady flows.

Intramural and wall shear stress stimuli: general case. For quasi-steady-state fluid-solid-growth formulations modeled with the present framework, fluid and solid computations interact through the mechanobi- 
ologically equilibrium condition $\Upsilon_{h}^{\xi}=1$. From Eq. (75), considering also proportional out-of-equilibrium stimulus functions $\Upsilon^{\xi}-1$ for smooth muscle and collagen, such that $\eta_{K}=K_{\sigma}^{\xi} / K_{\tau}^{\xi}[7]$, we arrive at

$$
\frac{\sigma_{I h}-\sigma_{I o}}{\sigma_{I o}}=\frac{1}{\eta_{K}} \frac{\tau_{w h}-\tau_{w o}}{\tau_{w o}}
$$

From Eq. (32) we obtain $\sigma_{I h}=\sigma_{I h}^{x}-3 p_{h}$, which leads to

$$
p_{h}=\sigma_{v h}^{x}-\sigma_{v o}\left(1+\frac{1}{\eta_{K}}\left(\frac{\tau_{w h}}{\tau_{w o}}-1\right)\right)
$$

with the volumetric stresses $\sigma_{v o}=\frac{1}{3} \operatorname{tr} \sigma_{o}$ (known from the original homeostatic, loaded state) and $\sigma_{v h}^{x}=\frac{1}{3} \operatorname{tr} \boldsymbol{\sigma}_{h}^{x}$. Importantly, note that Eq. (78) couples solid stress computations, through Eq. (32), with blood flow computations, through the wall shear stress measure $\tau_{w h}$. In particular, the volumetric Cauchy stress for the mixture $\sigma_{v h}=\sigma_{v h}^{x}-p_{h}$ in Eq. (32) reads

$$
\sigma_{v h}=\sigma_{v o}\left(1+\frac{1}{\eta_{K}}\left(\frac{\tau_{w h}}{\tau_{w o}}-1\right)\right)
$$

where, consistent with the considered stimulus function, the flow-induced wall shear stress modulates the intramural volumetric stress.

Intramural and wall shear stress stimuli: cylindrical arterial segments. Consider a long cylindrical artery that remains cylindrical over G\&R time scales, for which the wall shear stress expression $\tau_{w}=4 \mu Q / \pi a^{3}$ applies. Yet, this analytical expression for $\tau_{w}$ cannot be assessed locally in our arterial wall model because of the presence of the luminal radius $a$, which represents a geometrical outcome of the boundary value problem (i.e., the computed radial coordinate of the inner surface). However, we can relate the global variable $a$ with variables defined locally within a cylindrical arterial wall, at a given current radial coordinate $r$, satisfying $a \leq r \leq r_{\text {out }}$, with $r_{\text {out }}$ the current outer radius, as

$$
a=r-(r-a)=r_{o} \frac{r}{r_{o}}-\left(r_{o}-a_{o}\right) \frac{r-a}{r_{o}-a_{o}} \simeq r_{o} \lambda_{\theta}-\left(r_{o}-a_{o}\right) \lambda_{r}
$$

where $r_{o}$ is the original radial coordinate, $r / r_{o}=\lambda_{\theta}$ is the (exact) local circumferential stretch computed from $\mathbf{C}=\mathbf{F}^{\mathrm{T}} \mathbf{F}$ as $\lambda_{\theta}=\sqrt{\mathbf{C}: \mathbf{e}_{\theta} \otimes \mathbf{e}_{\theta}}$, and $(r-a) /\left(r_{o}-a_{o}\right) \simeq \lambda_{r}$ is an (approximated) local radial stretch computed as $\lambda_{r}=\sqrt{\mathbf{C}: \mathbf{e}_{r} \otimes \mathbf{e}_{r}}$, with $\mathbf{e}_{\theta}$ and $\mathbf{e}_{r}$ unit vectors along circumferential and radial directions. With $\tau_{w h} / \tau_{w o}=Q_{h} a_{o}^{3} / Q_{o} a_{h}^{3}$, Eq. (78) becomes

$$
p_{h}=\sigma_{v h}^{x}-\sigma_{v o}\left(1+\frac{1}{\eta_{K}}\left(\frac{Q_{h} / Q_{o}}{\left(\left(r_{o} / a_{o}\right) \lambda_{\theta}-\left(r_{o} / a_{o}-1\right) \lambda_{r}\right)^{3}}-1\right)\right)
$$

which, compared to Eq. (36), requires additional straightforward tangent contributions in Eq. expressed in terms of the derivatives

$$
2 \frac{\partial \lambda_{i}}{\partial \mathbf{C}}=2 \frac{\partial \sqrt{\mathbf{C}: \mathbf{e}_{i} \otimes \mathbf{e}_{i}}}{\partial\left(\mathbf{C}: \mathbf{e}_{i} \otimes \mathbf{e}_{i}\right)} \frac{\partial\left(\mathbf{C}: \mathbf{e}_{i} \otimes \mathbf{e}_{i}\right)}{\partial \mathbf{C}}=\frac{1}{\lambda_{i}} \mathbf{e}_{i} \otimes \mathbf{e}_{i}
$$

for both $i=r, \theta$, and that we used to illustrate qualitative results for arterial G\&R in examples above.

\section{Appendix D. Derivation of tangent moduli contribution $\hat{\sigma}_{\mathrm{N}}^{\xi}: 2 \partial\left(\mathrm{U}_{h}^{-1} \odot \mathrm{U}_{h}^{-1}\right) / \partial \mathrm{C}_{h}$}

We derive here the expression for a fourth-order tensor $\mathbb{C}_{\hat{\boldsymbol{\sigma}}}^{\xi}=\hat{\boldsymbol{\sigma}}_{\mathrm{N}}^{\xi}: 2 \partial\left(\mathbf{U}_{h}^{-1} \odot \mathbf{U}_{h}^{-1}\right) / \partial \mathbf{C}_{h}$ based on spectral decompositions for the rate of change of the different tensors involved [61,62]. For notation 
simplicity, we disregard subscript $h$ in deformation tensors. The spectral decomposition of the right Cauchy-Green tensor $\mathbf{C}$ reads

$$
\mathbf{C}=\sum_{i=1}^{3} \lambda_{i}^{2} \mathbf{N}_{i} \otimes \mathbf{N}_{i}=\sum_{i=1}^{3} \lambda_{i}^{2} \mathbf{N}_{i i}
$$

where $\lambda_{i}$ are stretches and $\mathbf{N}_{i}$ Lagrangian strain eigenvectors, and we define eigentensors $\mathbf{N}_{a b \ldots c}:=$ $\mathbf{N}_{a} \otimes \mathbf{N}_{b} \otimes \ldots \otimes \mathbf{N}_{c}$. The material time derivative of $\dot{\mathbf{C}}$ yields

$$
\dot{\mathbf{C}}=\sum_{i=1}^{3} 2 \lambda_{i} \dot{\lambda}_{i} \mathbf{N}_{i i}+\sum_{i=1}^{3} \sum_{j \neq i}\left(\lambda_{j}^{2}-\lambda_{i}^{2}\right) \Omega_{i j} \mathbf{N}_{i j}
$$

where $\dot{\mathbf{N}}_{i i}=\dot{\mathbf{N}}_{i} \otimes \mathbf{N}_{i}+\mathbf{N}_{i} \otimes \dot{\mathbf{N}}_{i}$ and $\Omega_{i j}=-\Omega_{j i}$ are components of the angular velocity (skew) tensor of Lagrangian eigenvectors $\boldsymbol{\Omega}$ expressed in that same basis, such that

$$
\dot{\mathbf{N}}_{i}=\mathbf{\Omega} \mathbf{N}_{i}=\sum_{j=1}^{3} \Omega_{j i} \mathbf{N}_{j}=\sum_{j=1, j \neq i}^{3} \Omega_{j i} \mathbf{N}_{j}=: \sum_{j \neq i} \Omega_{j i} \mathbf{N}_{j} .
$$

Consider now the spectral decomposition of the inverse of the right stretch tensor

$$
\mathbf{U}^{-1}=\sum_{i=1}^{3} \frac{1}{\lambda_{i}} \mathbf{N}_{i} \otimes \mathbf{N}_{i}=\sum_{i=1}^{3} \frac{1}{\lambda_{i}} \mathbf{N}_{i i}
$$

The spectral decomposition of the fourth-order tensor $\mathbf{U}^{-1} \odot \mathbf{U}^{-1}$ is

$$
\mathbf{U}^{-1} \odot \mathbf{U}^{-1}=\sum_{i=1}^{3} \sum_{j=1}^{3} \frac{1}{\lambda_{i} \lambda_{j}} \mathbf{N}_{i i} \odot \mathbf{N}_{j j}=\sum_{i=1}^{3} \sum_{j=1}^{3} \frac{1}{\lambda_{i} \lambda_{j}} \mathbf{N}_{i j i j}
$$

Its material time derivative, proceeding similarly as we did for $\dot{\mathbf{C}}$, and after few algebraic (index) manipulations, reads

$$
\overline{\mathbf{U}^{-1} \odot \mathbf{U}^{-1}}=-\sum_{i=1}^{3} \sum_{j=1}^{3} \frac{\dot{\lambda}_{i}}{\lambda_{i}^{2} \lambda_{j}}\left(\mathbf{N}_{i j i j}+\mathbf{N}_{j i j i}\right)+\sum_{i=1}^{3} \sum_{j=1}^{3} \sum_{k \neq i} \Omega_{i k}\left(\frac{1}{\lambda_{k} \lambda_{j}}-\frac{1}{\lambda_{i} \lambda_{j}}\right)\left(\mathbf{N}_{i j k j}+\mathbf{N}_{j i j k}\right) .
$$

Since $\overline{\mathbf{U}^{-1} \odot \mathbf{U}^{-1}}=\frac{\partial\left(\mathbf{U}^{-1} \odot \mathbf{U}^{-1}\right)}{\partial \mathbf{C}}: \dot{\mathbf{C}}$, by comparing expressions for $\dot{\mathbf{C}}$ and $\overline{\mathbf{U}^{-1} \odot \mathbf{U}^{-1}}$, one identifies the sixth-order tensor

$$
\begin{aligned}
\frac{\partial\left(\mathbf{U}^{-1} \odot \mathbf{U}^{-1}\right)}{\partial \mathbf{C}}= & -\sum_{i=1}^{3} \sum_{j=1}^{3} \frac{1}{2 \lambda_{i}^{3} \lambda_{j}}\left(\mathbf{N}_{i j i j i i}+\mathbf{N}_{j i j i i i}\right) \\
& -\sum_{i=1}^{3} \sum_{j=1}^{3} \sum_{k \neq i} \frac{1}{2 \lambda_{i} \lambda_{j} \lambda_{k}} \frac{1}{\lambda_{i}+\lambda_{k}}\left(\mathbf{N}_{i j k j i k}+\mathbf{N}_{i j k j k i}+\mathbf{N}_{j i j k i k}+\mathbf{N}_{j i j k k i}\right)
\end{aligned}
$$

which could be implemented, as is, in finite element codes to be subsequently doubly contracted, numerically, with $2 \hat{\boldsymbol{\sigma}}_{\mathrm{N}}^{\xi}$ to give $\mathbb{C}_{\hat{\boldsymbol{\sigma}}}^{\xi}$. More efficiently, however, we perform the double contraction analytically, and the result is implemented directly as a fourth-order tensor in our user-defined material subroutine. Consider then the spectral decomposition for the rotated stress tensor $\hat{\sigma}_{\mathrm{N}}^{\xi}$ in terms of the eigentensors 
$\mathbf{N}_{i j}$, which are not coaxial, in general, thereby

$$
\hat{\boldsymbol{\sigma}}_{\mathrm{N}}^{\xi}=\sum_{i=1}^{3} \sum_{j=1}^{3} \hat{\sigma}_{i j}^{\xi} \mathbf{N}_{i j}
$$

from which $\mathbb{C}_{\hat{\sigma}}^{\xi}$ is obtained as

$$
\mathbb{C}_{\hat{\boldsymbol{\sigma}}}^{\xi}=-\sum_{i=1}^{3} \sum_{j=1}^{3} \frac{\hat{\sigma}_{i j}^{\xi}}{\lambda_{i}^{3} \lambda_{j}}\left(\mathbf{N}_{i j i i}+\mathbf{N}_{j i i i}\right)-\sum_{i=1}^{3} \sum_{j=1}^{3} \sum_{k \neq i} \frac{\hat{\sigma}_{i j}^{\xi}}{\lambda_{i} \lambda_{j} \lambda_{k}\left(\lambda_{i}+\lambda_{k}\right)}\left(\mathbf{N}_{k j i k}+\mathbf{N}_{k j k i}+\mathbf{N}_{j k i k}+\mathbf{N}_{j k k i}\right)
$$

Importantly, note that the fourth-order tensor $\mathbb{C}_{\hat{\boldsymbol{\sigma}}}^{\xi}$ preserves minor symmetries $(a b c d) \leftrightarrow(b a c d) \leftrightarrow(a b d c)$, but (generally) not the major symmetry $(a b c d) \leftrightarrow(c d a b)$.

\section{Appendix E. Specialization: an equivalent thin-walled artery}

We reformulate here the main algebraic equations derived in [7] to show their consistency with the general boundary value formulation above.

\section{Formulation in [7]}

We solve a system of nonlinear algebraic equations formed by mechanobiological equilibrium $\Upsilon_{h}=1$, the constraint $\sum \phi_{h}^{\alpha}=\rho_{h}^{\alpha} / \rho=1$, and mechanical equilibrium along both (in-plane) circumferential and axial directions of a cylindrical artery, namely

$$
\begin{aligned}
\Upsilon_{h} & =1+K_{\sigma}\left(\frac{\sigma_{\theta \theta h}+\sigma_{z z h}}{\sigma_{\theta \theta o}+\sigma_{z z o}}-1\right)-K_{\tau}\left(\frac{Q_{h} a_{o}^{3}}{Q_{o} a_{h}^{3}}-1\right)=1 \\
\rho & =\rho_{h}^{e}+\rho_{h}^{m}+\rho_{h}^{c} \\
\sigma_{\theta \theta h} & =\sum_{\alpha}^{m, c} \frac{\rho_{h}^{\alpha}}{\rho} \hat{\sigma}_{\theta \theta}^{\alpha}+\frac{\rho_{h}^{e}}{\rho} \hat{S}_{\theta \theta h}^{e} G_{\theta}^{e 2} \lambda_{\theta h}^{2}+\frac{\rho_{h}^{m}}{\rho} \hat{\sigma}_{\theta \theta h}^{a c t}-\frac{\rho_{h}^{e}}{\rho} \hat{S}_{r r h}^{e} G_{r}^{e 2} \lambda_{r h}^{2}=\frac{P_{h} a_{h}}{h_{h}} \\
\sigma_{z z h} & =\sum_{\alpha}^{m, c} \frac{\rho_{h}^{\alpha}}{\rho} \hat{\sigma}_{z z}^{\alpha}+\frac{\rho_{h}^{e}}{\rho} \hat{S}_{z z h}^{e} G_{z}^{e 2} \lambda_{z h}^{2}-\frac{\rho_{h}^{e}}{\rho} \hat{S}_{r r h}^{e} G_{r}^{e 2} \lambda_{r h}^{2}=\frac{f_{z h}}{\pi h_{h}\left(2 a_{h}+h_{h}\right)}
\end{aligned}
$$

where the primary unknowns are $a_{h}, h_{h}, \rho_{h}^{c}$, and $f_{z h}$. In particular, the evolved homeostatic Lagrange multiplier $p_{h}$ (not shown) is obtained from the (not shown) radial equilibrium equation and associated boundary condition $\left(\sigma_{r r h}=0\right)$, which enables substitution of $p_{h}$ in expressions for in-plane stresses, hence, representing a specific resolution procedure for a cylindrical artery.

\section{An equivalent formulation aimed for finite element implementation}

One can alternatively solve an equivalent system of nonlinear algebraic equations formed by mechanobiological equilibrium $\Upsilon_{h}=1$ plus mechanical equilibrium along the (out-of- and in-plane) radial, circum- 
ferential, and axial directions, namely

$$
\begin{aligned}
\Upsilon_{h} & =1+K_{\sigma}\left(\frac{\sigma_{r r h}+\sigma_{\theta \theta h}+\sigma_{z z h}}{\sigma_{r r o}+\sigma_{\theta \theta o}+\sigma_{z z o}}-1\right)-K_{\tau}\left(\frac{Q_{h} a_{o}^{3}}{Q_{o} a_{h}^{3}}-1\right)=1 \\
\sigma_{r r h} & =\sum_{\alpha}^{e, m, c} \sigma_{r r h}^{\alpha}-p_{h}=\frac{\rho_{h}^{e}}{\rho} \hat{S}_{r r h}^{e} G_{r}^{e 2} \lambda_{r h}^{2}-p_{h}=-\frac{P_{h}}{2} \\
\sigma_{\theta \theta h} & =\sum_{\alpha}^{e, m, c} \sigma_{\theta \theta h}^{\alpha}-p_{h}=\sum_{\alpha}^{m, c} \frac{\rho_{h}^{\alpha}}{\rho} \hat{\sigma}_{\theta \theta}^{\alpha}+\frac{\rho_{h}^{e}}{\rho} \hat{S}_{\theta \theta h}^{e} G_{\theta}^{e 2} \lambda_{\theta h}^{2}+\frac{\rho_{h}^{m}}{\rho} \hat{\sigma}_{\theta \theta h}^{a c t}-p_{h}=\frac{P_{h} a_{h}}{h_{h}} \\
\sigma_{z z h} & =\sum_{\alpha}^{e, m, c} \sigma_{z z h}^{\alpha}-p_{h}=\sum_{\alpha}^{m, c} \frac{\rho_{h}^{\alpha}}{\rho} \hat{\sigma}_{z z}^{\alpha}+\frac{\rho_{h}^{e}}{\rho} \hat{S}_{z z h}^{e} G_{z}^{e 2} \lambda_{z h}^{2}-p_{h}=\frac{f_{z h}}{\pi h_{h}\left(2 a_{h}+h_{h}\right)}
\end{aligned}
$$

where the primary unknowns are $a_{h}, h_{h}, p_{h}$, and $f_{z h}$, noting in Eqs. (87) and (88) additionally the consideration of a mean radial stress $-P_{h} / 2$ for more accurate comparisons with FE analyses. In particular, the evolved homeostatic Lagrange multiplier $p_{h}$ can be obtained in this case from the mechanobiological equilibrium equation (recall Eq. (33)), which enables substitution of $p_{h}$ in expressions for out-of- and in-plane stresses (recall Eq. (32)), hence, representing a generalized resolution procedure for a cylindrical artery consistent with the general formulation derived above. 


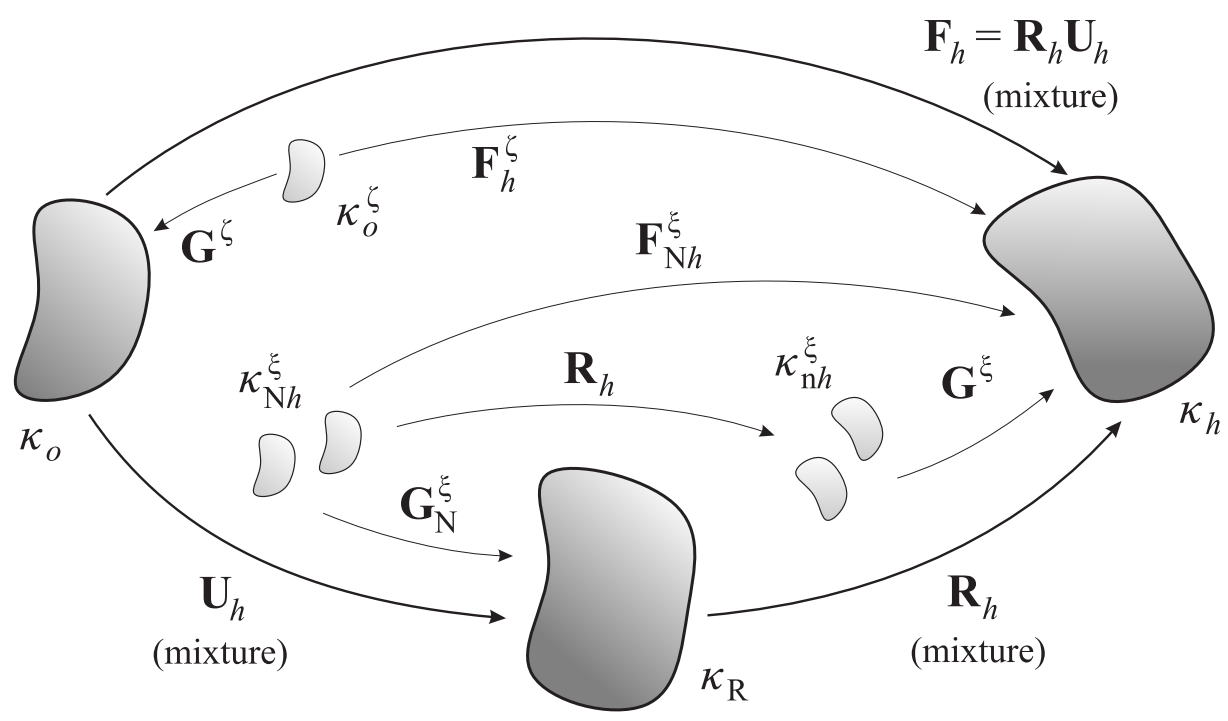

Figure 1: Schematic representation of different configurations $\kappa_{i}$ involved in mechanobiologically equilibrated arterial G\&R, with $\xi \equiv$ collagen (c) and smooth muscle $(\mathrm{m})$ experiencing continuous turnover but $\zeta \equiv$ elastin (e) not turning over. Note that $\kappa_{o}$ and $\kappa_{h}$ are both homeostatic; all configurations are in vivo. 


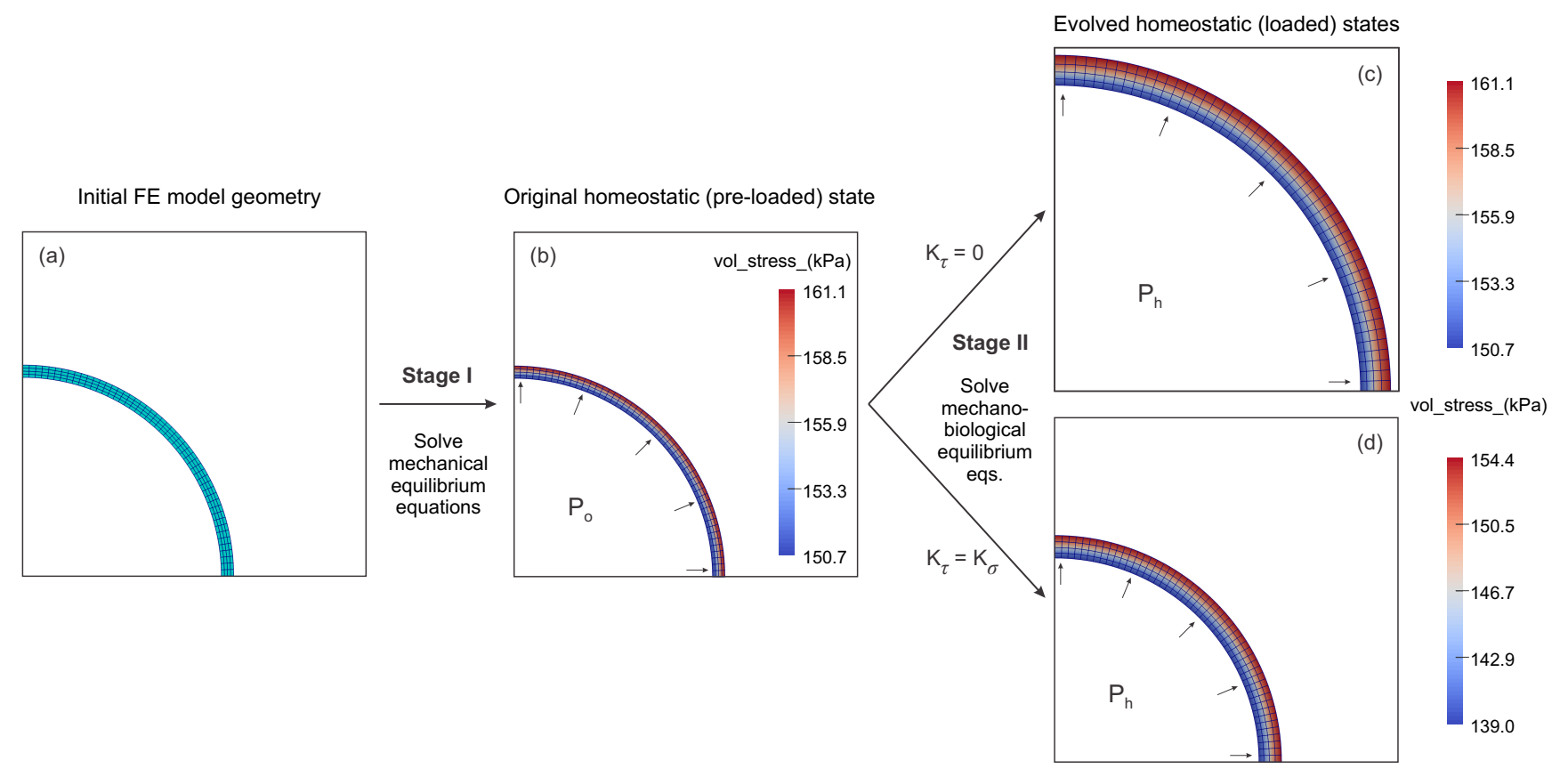

Figure 2: Initial (a) and computed after Stage I (b) quarter cross-section geometries (and meshes) for the initially cylindrical arterial segment considered in all FE simulations. Shown in (b), too, is the volumetric stress contour plot for this triaxially pre-stressed state consistent with parameters in Table 1 , inner pressure $P_{o}=13.98 \mathrm{kPa}$, flow rate ratio $\epsilon_{o}=1$, and (implicit, with respect to an unloaded state) in vivo axial stretch $\lambda_{z}^{\mathrm{iv}}=1.62$. In addition, deformed cross-sections and volumetric stress contour plots are shown for increases in pressure up to $P_{h}=1.6 P_{o}$ without (panel c, $K_{\tau} / K_{\sigma}=0$ ) or with (panel d, $K_{\tau} / K_{\sigma}=1$ ) flow-induced shear stress effects on G\&R (see Appendix C). Note the excessive dilation and thickening for the simulation (c) that preserves local values of volumetric stress with respect to (b) but neglects shear stress effects, see Eq. (35); in contrast, observe the mechano-adaptive thickening with little dilation (d), with slightly decreased volumetric stresses consistent with shear stresses reduced with respect to (b), see Eq. (79). Shown are smoothed stresses computed from (constant, but monotonic through the thickness) elemental stresses. 
bioRxiv preprint doi: https://doi.org/10.1101/2020.02.27.968768; this version posted May 9, 2020. The copyright holder for this preprint (which was not certified by peer review) is the author/funder. All rights reserved. No reuse allowed without permission.
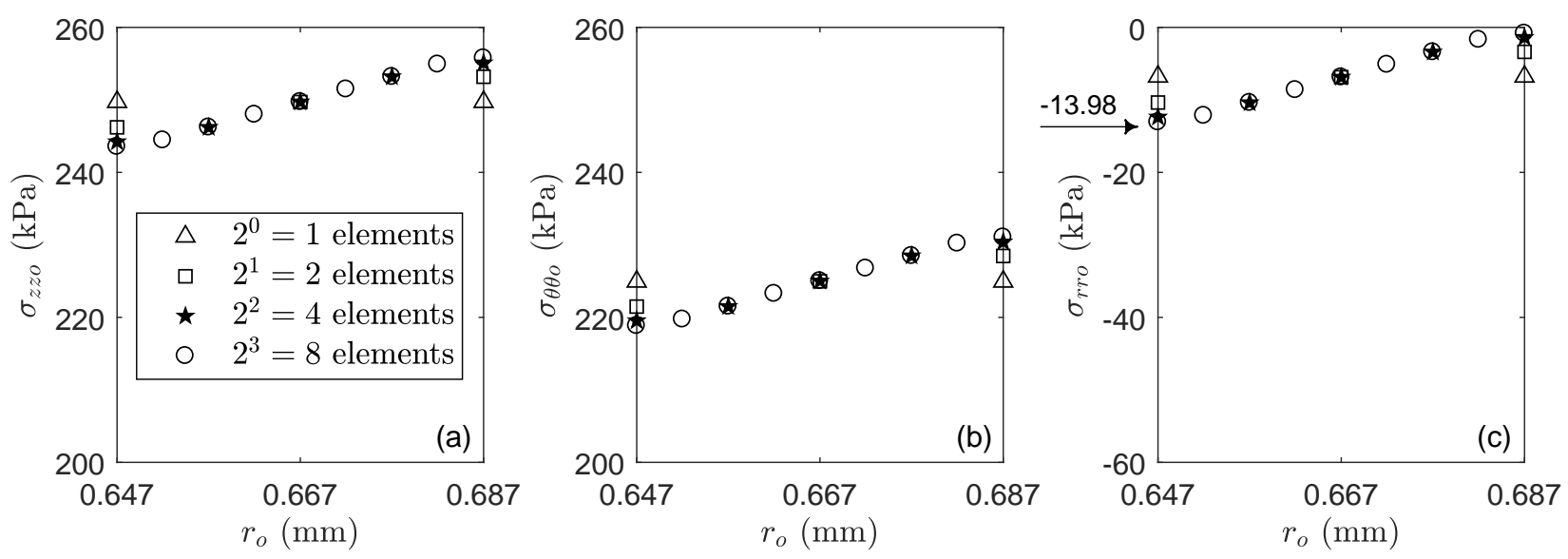

Figure 3: Mesh convergence study during the Stage I computation of the original pre-stressed homeostatic configuration as a function of the number of elements through the thickness of a mouse DTA $\left(a_{o} \leq r_{o} \leq h_{o}\right.$, Table 1$)$ at a homeostatic state $\left(P_{o}=13.98 \mathrm{kPa}\right.$, denoted by an arrow in panel $\mathrm{c}$, with $\left.\lambda_{z}^{\mathrm{iv}}=1.62\right)$. Four transmural elements were sufficient for this uni-layered wall model and thus were used in all subsequent simulations. Shown are axial (a), circumferential (b), and radial (c) Cauchy stresses at nodes interpolated from (constant, but monotonic through the thickness) elemental stresses. Note the different scales on the ordinates for the 40-micron thick wall. 

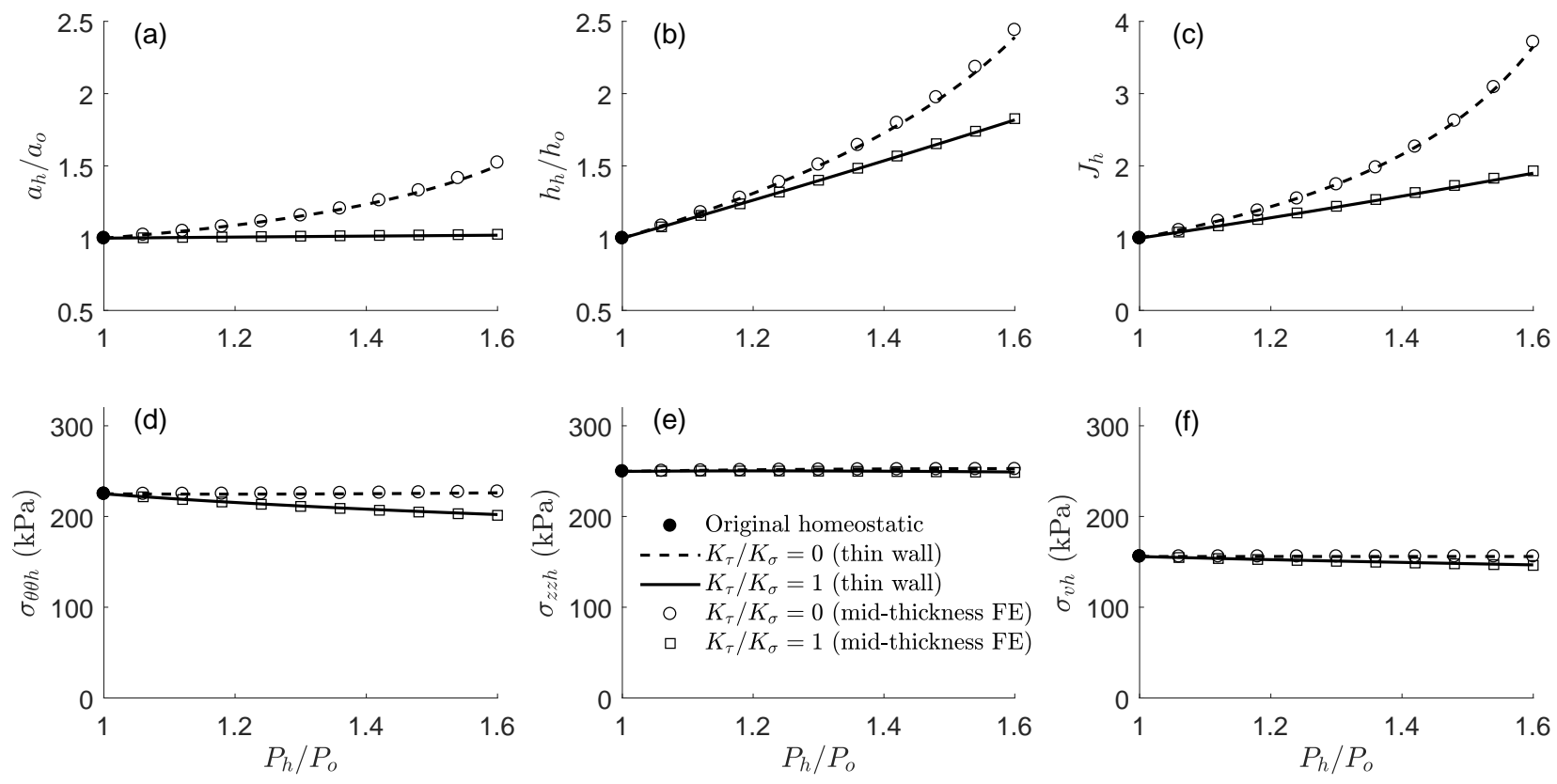

Figure 4: Analytical (thin-wall; lines) versus finite element (cylindrical model at mid-thickness; symbols) pressure-driven quasi-static G\&R calculations for increases in pressure from $P_{h}=P_{o}$ to $P_{h}=1.6 P_{o}$ without (dashed line and open circles, $K_{\tau} / K_{\sigma}=0$ ) or with (solid line and open squares, $K_{\tau} / K_{\sigma}=1$ ) shear stress effects on G\&R (see associated meshes and contour plots for $P_{h} / P_{o}=1.6$ in Figure 2). 

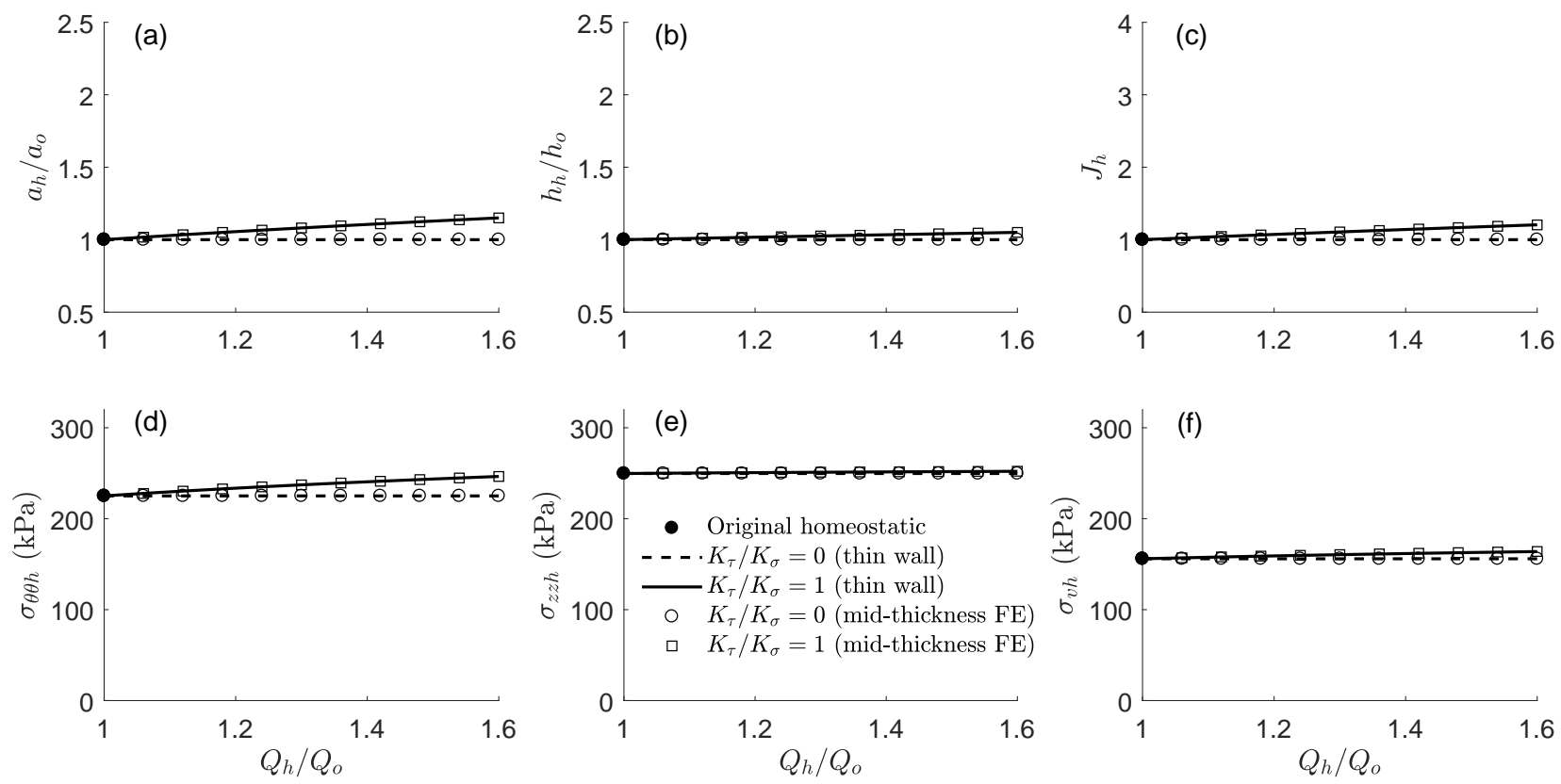

Figure 5: Analytical (thin-wall; lines) versus finite element (cylindrical model at mid-thickness; symbols) flow-driven quasistatic G\&R calculations for increases in blood flow rate from $Q_{h}=Q_{o}$ to $Q_{h}=1.6 Q_{o}$ without (dashed line and open circles, $K_{\tau} / K_{\sigma}=0$ ) or with (solid line and open squares, $K_{\tau} / K_{\sigma}=1$ ) shear stress effects on G\&R. 

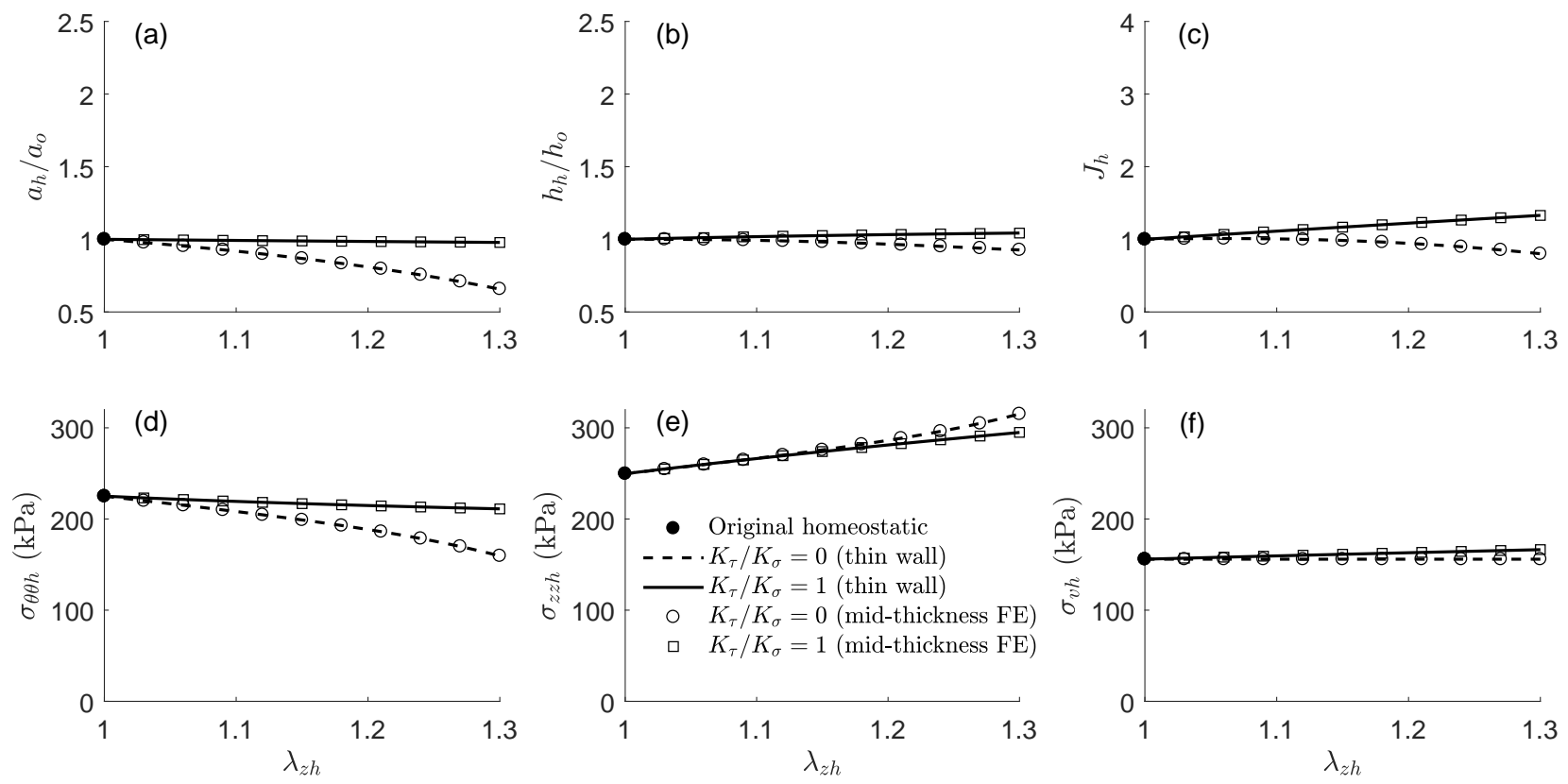

Figure 6: Analytical (thin-wall; lines) versus finite element (cylindrical model at mid-thickness; symbols) axial stretch-driven quasi-static G\&R calculations for increases in axial stretch from $\lambda_{h}=\lambda_{o}=1$ to $\lambda_{h}=1.3 \lambda_{o}=1.3$ without (dashed line and open circles, $K_{\tau} / K_{\sigma}=0$ ) or with (solid line and open squares, $K_{\tau} / K_{\sigma}=1$ ) shear stress effects on G\&R. Note the increase in axial stress contributed by the elastically stretched elastin. 

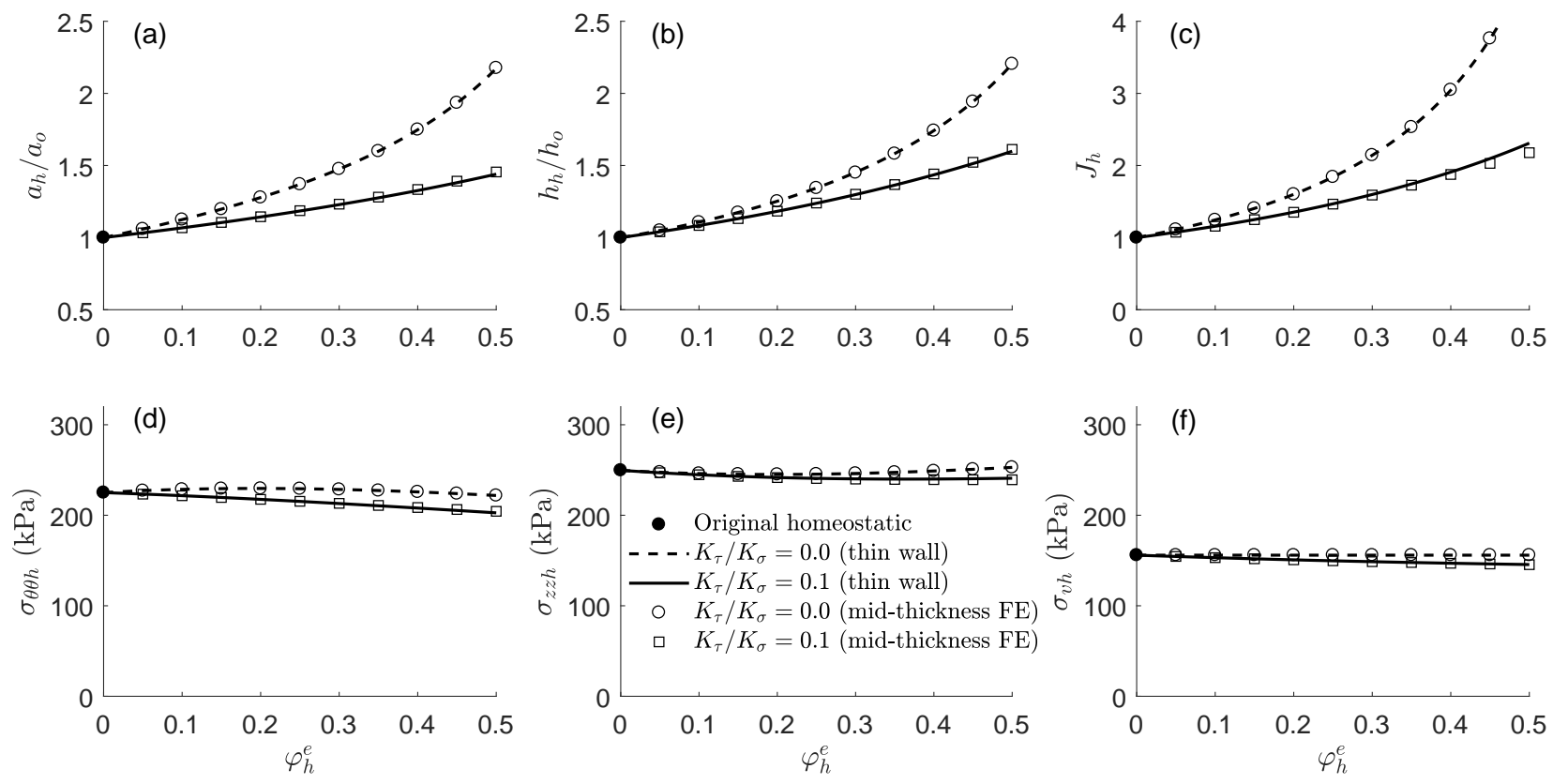

Figure 7: Analytical (thin-wall; lines) versus finite element (cylindrical model at mid-thickness; symbols) elastin degradationdriven quasi-static G\&R calculations for increases in the degradation parameter $\varphi_{h}^{e}=\left(c_{o}^{e}-c_{h}^{e}\right) / c_{o}^{e}$ from $\varphi_{h}^{e}=\varphi_{o}^{e}=0$ up to $\varphi_{h}^{e}=0.5$, while preserving inner pressure $P_{h}=P_{o}$, without (dashed line and open circles, $K_{\tau} / K_{\sigma}=0$ ) or with slight (solid line and open squares, $\left.K_{\tau} / K_{\sigma}=0.1\right)$ shear stress effects on G\&R. Both models predict asymptotic thickening and dilation with moderate change in biaxial stress for $\varphi_{h}^{e} \rightarrow 0.725$ when $K_{\tau} / K_{\sigma}=0$ (not shown), cf. [8]. 



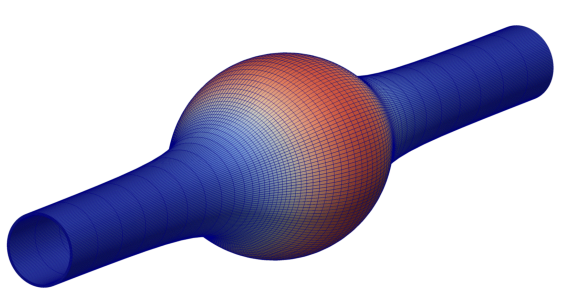

(a)
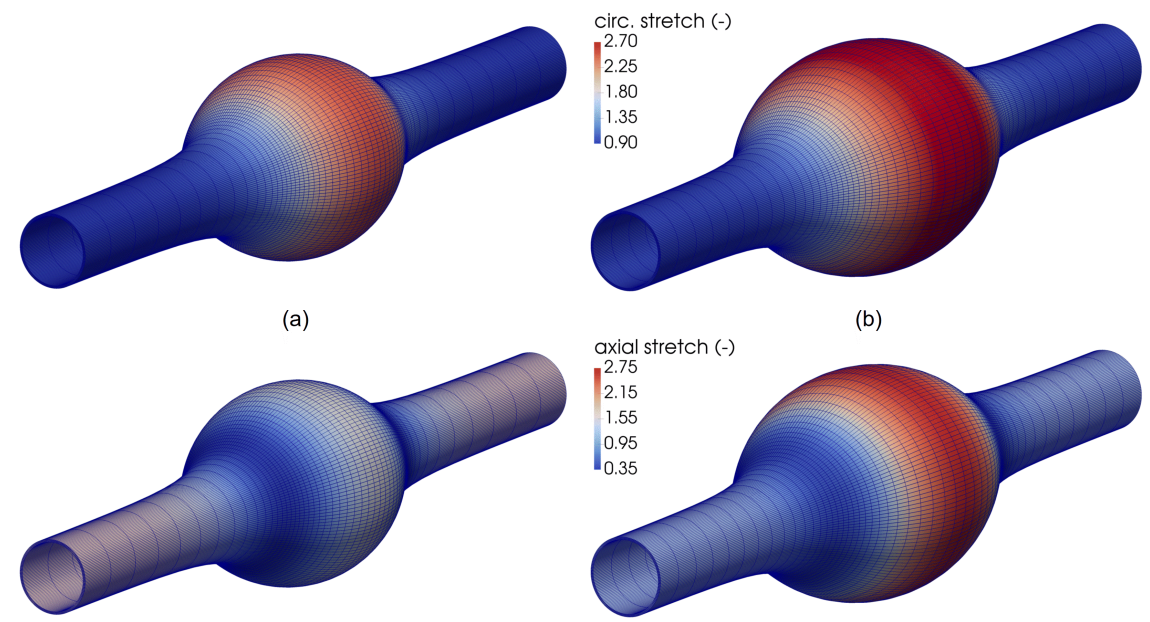

(b)

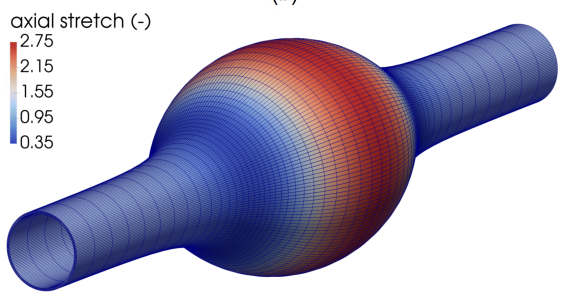

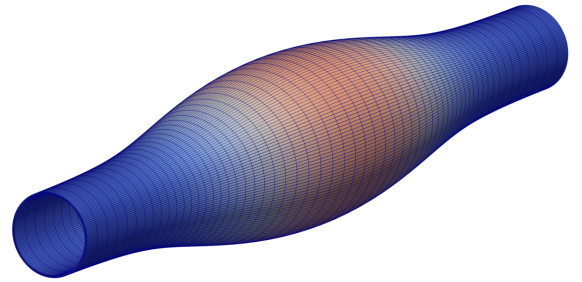

(c)

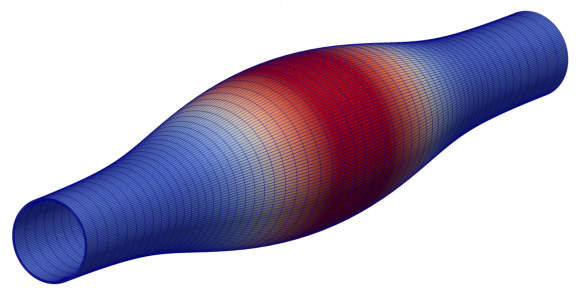

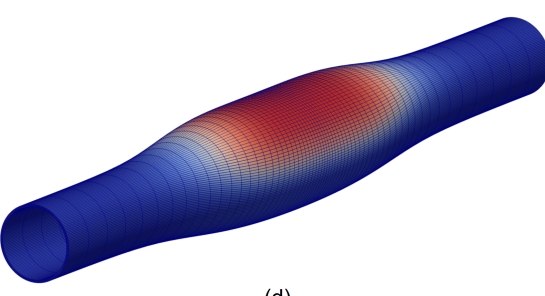

(d)

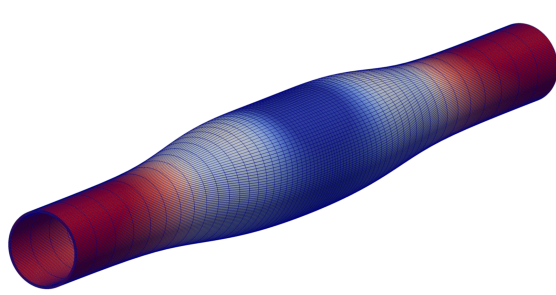

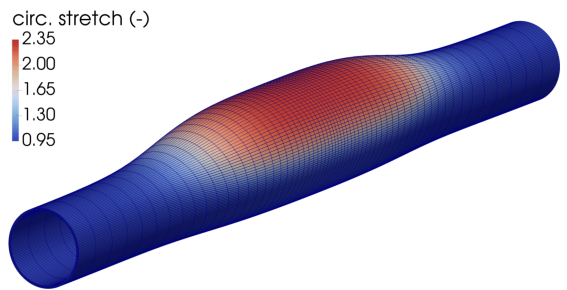

(e)

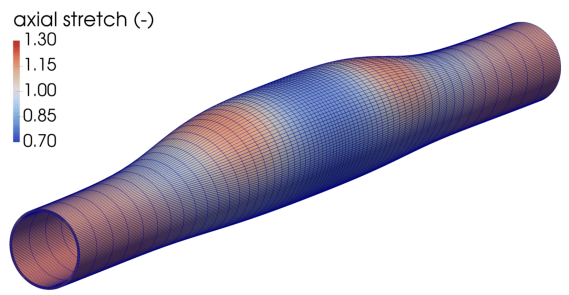

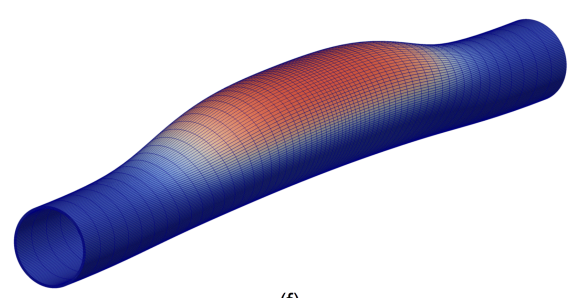

(f)

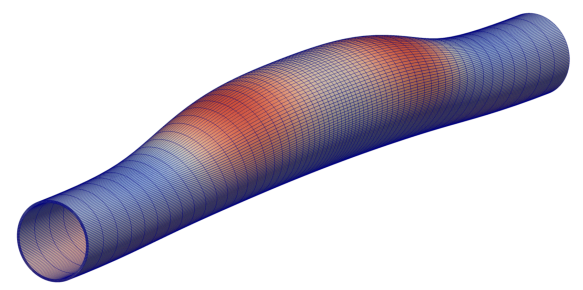

Figure 9: Mechanobiologically equilibrated states for an arterial segment under normotensive conditions $\left(P_{h}=P_{o}\right)$ with axisymmetrically $(\mathrm{a}, \mathrm{b}, \mathrm{c})$ or asymmetrically $(\mathrm{d}, \mathrm{e}, \mathrm{f})$ prescribed elastin damage $\left(\varphi_{h \mathrm{~m}}^{e}=0.65\right.$ for all cases $)$ in combination with gradually increased orientations of diagonal collagen fibers towards the circumferential direction as $(\mathrm{a}, \mathrm{d}) \alpha_{0 h}=\alpha_{0 o} \approx 30^{\circ},(\mathrm{b}$, e) $\alpha_{0 h}=(4 / 3) \alpha_{0 o} \approx 40^{\circ}$, and (c, f) $\alpha_{0 h}=(5 / 3) \alpha_{0 o} \approx 50^{\circ}$. Shown are deformed meshes and contour plots of circumferential (first and third rows) and axial (second and fourth rows) stretches with respect to an initially straight arterial segment under homeostatic conditions (see mesh (a) in Figure 8). Additional parameters that define the spatially distributed material insults and properties for the axisymmetric or asymmetric aneurysms are the same as described for simulations (b) or (d) in Figure 8. Note the increase in local axial growth within the damaged regions for an increased reorientation of diagonal fibers towards the circumferential direction for both axisymmetric and asymmetric aneurysms (from left to right). 
Box 1: Mechanobiologically equilibrated constrained mixture model for G\&R: kinematics and stresses

(i) Deformation gradient for the mixture (from $\kappa_{o}$ to $\kappa_{h}$, see Fig. 1)

$\mathbf{F}_{h}=\mathbf{R}_{h} \mathbf{U}_{h}$, with associated right Cauchy-Green tensor $\mathbf{C}_{h}=\mathbf{F}_{h}^{\mathrm{T}} \mathbf{F}_{h}$

(ii) Deformation gradient for constituents $\zeta$ (from $\kappa_{\mathrm{N}}^{\zeta}=\kappa_{\mathrm{n}}^{\zeta}$ to $\kappa_{h}$ ), which do not turnover

$\mathbf{F}_{h}^{\zeta}=\mathbf{F}_{h} \mathbf{G}^{\zeta}$, with associated right Cauchy-Green tensor $\mathbf{C}_{h}^{\zeta}=\mathbf{F}_{h}^{\zeta \mathrm{T}} \mathbf{F}_{h}^{\zeta}=\mathbf{G}^{\zeta} \mathbf{C}_{h} \mathbf{G}^{\zeta}$

(iii) Deformation gradient for constituents $\xi$ (from $\kappa_{\mathrm{N}}^{\xi} \neq \kappa_{\mathrm{n}}^{\xi}$ to $\kappa_{h}$ ), which turnover

$\mathbf{F}_{\mathrm{N} h}^{\xi}=\mathbf{R}_{h} \mathbf{G}_{\mathrm{N}}^{\xi}$, with associated right Cauchy-Green tensors $\mathbf{C}_{\mathrm{N} h}^{\xi}=\mathbf{F}_{\mathrm{N} h}^{\xi \mathrm{T}} \mathbf{F}_{\mathrm{N} h}^{\xi}=\mathbf{G}_{\mathrm{N}}^{\xi 2}$

(iv) Jacobian $\left(J_{h}=\operatorname{det} \mathbf{F}_{h}\right)$ dependent mass fractions for different constituents

$\phi_{h}^{\zeta}\left(J_{h}\right)=\frac{\phi_{o}^{\zeta}}{J_{h}} ; \phi_{h}^{\xi}\left(J_{h}\right)$ determined implicitly from Eqs. (11) and (12)

(v) Second Piola-Kirchhoff stresses for constituents $\zeta$ (at mixture-level)

$$
\mathbf{S}_{h}^{\zeta}=J_{h} \phi_{h}^{\zeta} \mathbf{G}^{\zeta} \hat{\mathbf{S}}_{h}^{\zeta} \mathbf{G}^{\zeta}=\phi_{o}^{\zeta} \mathbf{G}^{\zeta} \hat{\mathbf{S}}_{h}^{\zeta} \mathbf{G}^{\zeta}, \text { with constituent-level stresses } \hat{\mathbf{S}}_{h}^{\zeta}=\left.\frac{\partial \hat{W}^{\zeta}\left(\mathbf{C}^{\zeta}\right)}{\partial \mathbf{C}^{\zeta}}\right|_{\mathbf{C}_{h}^{\zeta}}
$$

(vi) Second Piola-Kirchhoff stresses for constituents $\xi$ (at mixture-level)

$$
\mathbf{S}_{h}^{\xi}=J_{h} \phi_{h}^{\xi} \mathbf{U}_{h}^{-1} \mathbf{G}_{\mathrm{N}}^{\xi} \hat{\mathbf{S}}_{\mathrm{N}}^{\xi} \mathbf{G}_{\mathrm{N}}^{\xi} \mathbf{U}_{h}^{-1} \text {, with constituent-level stresses } \hat{\mathbf{S}}_{\mathrm{N}}^{\xi}=\left.\frac{\partial \hat{W}^{\xi}\left(\mathbf{C}_{\mathrm{N}}^{\xi}\right)}{\partial \mathbf{C}_{\mathrm{N}}^{\xi}}\right|_{\mathbf{C}_{\mathrm{N} h}^{\xi}}
$$

(vii) Cauchy stresses for different constituents (at mixture-level)

$$
\left.\boldsymbol{\sigma}_{h}^{\zeta}=\phi_{h}^{\zeta} \mathbf{F}_{h}^{\zeta} \hat{\mathbf{S}}_{h}^{\zeta} \mathbf{F}_{h}^{\zeta \mathrm{T}} ; \boldsymbol{\sigma}_{h}^{\xi}=\phi_{h}^{\xi} \mathbf{F}_{\mathrm{N} h}^{\xi} \hat{\mathbf{S}}_{\mathrm{N}}^{\xi} \mathbf{F}_{\mathrm{N} h}^{\xi}=\phi_{h}^{\xi} \mathbf{R}_{h} \hat{\boldsymbol{\sigma}}_{\mathrm{N}}^{\xi} \mathbf{R}_{h}^{\mathrm{T}} \text { (rotated stresses } \hat{\boldsymbol{\sigma}}_{\mathrm{N}}^{\xi}=\mathbf{G}_{\mathrm{N}}^{\xi} \hat{\mathbf{S}}_{\mathrm{N}}^{\xi} \mathbf{G}_{\mathrm{N}}^{\xi}=\mathbf{c o n s t}\right)
$$

(viii) Consistency parameter (i.e., evolving Lagrange multiplier) enforcing mechanobiological equilibrium

$$
\Upsilon_{h}=1 \stackrel{(\text { e.g. })}{\Longrightarrow} p_{h}=\sum_{\alpha=1}^{N} \sigma_{v h}^{\alpha}-\sigma_{v o}, \text { with volumetric stresses } \sigma_{v}=\frac{1}{3} \operatorname{tr} \boldsymbol{\sigma}
$$

(ix) Rule-of-mixtures stresses for the mixture

$$
\mathbf{S}_{h}=\mathbf{S}_{h}^{x}+\mathbf{S}_{h}^{p}=\sum_{\alpha=1}^{N} \mathbf{S}_{h}^{\alpha}-J_{h} p_{h} \mathbf{C}_{h}^{-1} ; \quad \boldsymbol{\sigma}_{h}=\frac{1}{J_{h}} \mathbf{F}_{h} \mathbf{S}_{h} \mathbf{F}_{h}^{\mathrm{T}}=\boldsymbol{\sigma}_{h}^{x}+\boldsymbol{\sigma}_{h}^{p}=\sum_{\alpha=1}^{N} \boldsymbol{\sigma}_{h}^{\alpha}-p_{h} \mathbf{I}
$$


Box 2: Mechanobiologically equilibrated constrained mixture model for G\&R: Consistent linearization

(i) Derivative of (referential) mass fractions for constituents $\xi$ with respect to $J_{h}$

$\frac{d\left(J_{h} \phi_{h}^{\xi}\right)}{d J_{h}}, \xi=1, \ldots, N^{\xi}$ determined explicitly from derivatives of Eqs. (11) and (12)

(ii) Constitutive tangent for constituents $\zeta$ (mixture-level), which do not turnover

$\mathbb{C}_{h}^{\zeta}=2 \frac{\partial \mathbf{S}_{h}^{\zeta}}{\partial \mathbf{C}_{h}}=\phi_{o}^{\zeta} \mathbf{G}^{\zeta} \odot \mathbf{G}^{\zeta}: \hat{\mathbb{C}}_{h}^{\zeta}: \mathbf{G}^{\zeta} \odot \mathbf{G}^{\zeta}$

with constituent-level tangent $\hat{\mathbb{C}}_{h}^{\zeta}=2 \frac{\partial \hat{\mathbf{S}}_{h}^{\zeta}}{\partial \mathbf{C}_{h}^{\zeta}}=4 \frac{\partial^{2} \hat{W}^{\zeta}\left(\mathbf{C}_{h}^{\zeta}\right)}{\partial \mathbf{C}_{h}^{\zeta} \otimes \partial \mathbf{C}_{h}^{\zeta}}$

(iii) Constitutive tangent for constituents $\xi$ (mixture-level), which turnover

$$
\begin{aligned}
& \mathbb{C}_{h}^{\xi}=2 \frac{\partial \mathbf{S}_{h}^{\xi}}{\partial \mathbf{C}_{h}}=\frac{1}{\phi_{h}^{\xi}} \frac{d\left(J_{h} \phi_{h}^{\xi}\right)}{d J_{h}} \mathbf{S}_{h}^{\xi} \otimes \mathbf{C}_{h}^{-1}+J_{h} \phi_{h}^{\xi} \mathbb{C}_{\hat{\boldsymbol{\sigma}}}^{\xi} \\
& \text { with } \mathbb{C}_{\hat{\boldsymbol{\sigma}}}^{\xi}=\hat{\boldsymbol{\sigma}}_{\mathrm{N}}^{\xi}: 2 \frac{\partial\left(\mathbf{U}_{h}^{-1} \odot \mathbf{U}_{h}^{-1}\right)}{\partial \mathbf{C}_{h}} \text { given in spectral form in Appendix D }
\end{aligned}
$$

(iv) Contribution from evolving $p_{h}$ enforcing mechanobiologically equilibrated evolution

$$
\dot{\Upsilon}_{h}=0 \stackrel{(\text { e.g. })}{\Longrightarrow} \mathbb{C}_{h}^{p}=J_{h} \sigma_{v o} \mathbf{C}_{h}^{-1} \otimes \mathbf{C}_{h}^{-1}-\frac{1}{3} \mathbf{C}_{h}^{-1} \otimes \mathbf{C}_{h}: \mathbb{C}_{h}^{x}-\frac{2}{3} \mathbf{C}_{h}^{-1} \otimes \mathbf{S}_{h}^{x}+2 J_{h} p_{h} \mathbf{C}_{h}^{-1} \odot \mathbf{C}_{h}^{-1}
$$

(v) Referential and spatial tangent tensors for the mixture

$$
\mathbb{C}_{h}=\mathbb{C}_{h}^{x}+\mathbb{C}_{h}^{p}=\sum_{\alpha=1}^{N} \mathbb{C}_{h}^{\alpha}+\mathbb{C}_{h}^{p} ; \quad \mathbb{C}_{h}=\frac{1}{J_{h}} \mathbf{F}_{h} \odot \mathbf{F}_{h}: \mathbb{C}_{h}: \mathbf{F}_{h}^{\mathrm{T}} \odot \mathbf{F}_{h}^{\mathrm{T}}=\mathbb{C}_{h}^{x}+\mathbb{C}_{h}^{p}
$$


Box 3: Two-stage algorithm to compute mechanobiologically equilibrated G\&R evolutions

\section{Stage I: Hyperelastic pre-loading to the original homeostatic state}

Given (properties defined pointwise, in general):

- Geometry, external loads, and boundary conditions at G\&R time $s=0$

- Mass fractions $\phi_{o}^{\alpha}$ for all constituents $\alpha$ at G\&R time $s=0$

- Deposition stretch tensors $\mathbf{G}^{\zeta}$ and $\mathbf{G}_{\mathrm{N}}^{\xi}$ for all constituents $\zeta$ and $\xi$ at G\&R time $s=0$

- Strain energy functions $\hat{W}^{\alpha}$ for all constituents $\alpha$

- Volumetric (penalty) strain energy function $U(\ln J)$ for the mixture

(I.1) Solve boundary value problem at $s=0$, with:

Stresses and constitutive tangents in Eqs. (49) and (50)

(I.2) Store at integration points (for the only time, for a smooth transition to Stage II):

$\mathbf{F}_{o} \simeq \mathbf{I}, \quad \sigma_{v o}=\frac{1}{3 J_{o}} \mathbf{S}_{o}: \mathbf{C}_{o}, \quad$ and $\quad \hat{\sigma}_{\mathrm{N}}^{\xi}=\frac{1}{J_{o} \phi_{o}^{\xi}} \mathbf{U}_{o} \mathbf{S}_{o}^{\xi} \mathbf{U}_{o} \simeq \mathbf{G}_{\mathrm{N}}^{\xi} \hat{\mathbf{S}}_{\mathrm{N}}^{\xi} \mathbf{G}_{\mathrm{N}}^{\xi} \quad\left(\xi=1, \ldots, N^{\xi}\right)$

\section{Stage II: Mechanobiologically equilibrated G\&R evolution}

Given, additionally (properties defined pointwise, in general):

- Change in external loads and/or other stimuli over G\&R time $s>0$

- $N^{\xi}-1$ independent gain-removal ratios $\eta^{i j}$ between constituents $\left\{\xi_{i}, \xi_{j}\right\}$

(II.1) Solve boundary value problem at each $s=s_{h}$, with:

Stresses and consistent tangents in Boxes 1 and 2 


\begin{tabular}{|c|c|c|}
\hline Inner Radius, Thickness, Length & $a_{o}, h_{o}, l_{o}$ & $0.647 \mathrm{~mm}, 0.040 \mathrm{~mm}, 15 \mathrm{~mm}$ \\
\hline Mass Fractions & $\phi_{o}^{e}, \phi_{o}^{m}, \phi_{o}^{c}$ & $0.34,0.33,0.33$ \\
\hline Collagen Fractions & $\beta^{\theta}, \beta^{z}, \beta^{d}$ & $0.056,0.067,0.877$ \\
\hline Diagonal Collagen Orientation & $\alpha_{0}$ & $29.91^{\circ}$ \\
\hline Elastin Parameter & $c^{e}$ & $89.71 \mathrm{kPa}$ \\
\hline Smooth Muscle Parameters & $c_{1}^{m}, c_{2}^{m}$ & $261.4 \mathrm{kPa}, 0.24$ \\
\hline Collagen Parameters & $c_{1}^{c}, c_{2}^{c}$ & $234.9 \mathrm{kPa}, 4.08$ \\
\hline Elastin Deposition Stretches & $G_{r}^{e}, G_{\theta}^{e}, G_{z}^{e}$ & $1 /\left(G_{\theta}^{e} G_{z}^{e}\right), 1.9,1.62$ \\
\hline Muscle/Collagen Deposition Stretches & $G^{m}, G^{c}$ & $1.20,1.25$ \\
\hline Combined Production-Removal & $\eta^{m c}=\left(k^{m} / k^{c}\right) \cdot\left(K^{m} / K^{c}\right)$ & 1 \\
\hline
\end{tabular}

Table 1: Representative baseline model parameters for a mouse descending thoracic aorta, assuming that elastin does not turnover during the G\&R period but smooth muscle and collagen turnover continuously with constant deposition stretches (adapted from original homeostatic parameters in [15], with $\eta^{m c}=1$ because gain and rate parameters were determined while including inflammatory effects, which we do not consider here). Collagen family mass fractions are defined by $\beta^{j}=\phi_{o}^{c j} / \phi_{o}^{c}$, with $j=\theta, z, d$ representing circumferential, axial, and symmetric diagonal directions. The angle $\alpha_{0}$ is defined with respect to the axial direction. Additional values for $\eta_{K}=K_{\sigma}^{\xi} / K_{\tau}^{\xi}$ in Eq. (77) are given in the examples when appropriate. 


\begin{tabular}{|c||c|c||c|c||c|c|}
\cline { 2 - 7 } \multicolumn{1}{c|}{} & \multicolumn{2}{c||}{$\mathrm{G \& R}\left(K_{\tau} / K_{\sigma}=0\right)$} & $\mathrm{G} \& \mathrm{R}\left(K_{\tau} / K_{\sigma}=1\right)$ & \multicolumn{2}{c|}{ Hyperelastic } \\
\cline { 2 - 7 } & force & energy & force & energy & force & energy \\
\hline \hline Iteration 0 & $2.9 \mathrm{E}+00$ & $1.5 \mathrm{E}-00$ & $2.6 \mathrm{E}+00$ & $9.0 \mathrm{E}-02$ & $6.5 \mathrm{E}+00$ & $5.1 \mathrm{E}-01$ \\
Iteration 1 & $2.2 \mathrm{E}-01$ & $9.7 \mathrm{E}-03$ & $3.3 \mathrm{E}-02$ & $5.1 \mathrm{E}-04$ & $7.3 \mathrm{E}+01$ & $6.8 \mathrm{E}-02$ \\
Iteration 2 & $1.5 \mathrm{E}-04$ & $1.4 \mathrm{E}-08$ & $1.2 \mathrm{E}-05$ & $4.3 \mathrm{E}-09$ & $4.9 \mathrm{E}-01$ & $2.3 \mathrm{E}-04$ \\
Iteration 3 & $6.1 \mathrm{E}-10$ & $1.7 \mathrm{E}-18$ & $3.2 \mathrm{E}-10$ & $3.6 \mathrm{E}-20$ & $9.4 \mathrm{E}-04$ & $4.7 \mathrm{E}-09$ \\
Iteration 4 & \multicolumn{3}{|c||}{$6.63 \mathrm{~s}$} & \multicolumn{2}{c||}{$6.76 \mathrm{~s}$} & \multicolumn{2}{c|}{$6.37 \mathrm{~s}$} \\
\hline \hline Time (1+10 steps) & \multicolumn{3}{c|}{$6.1 .7 \mathrm{E}-10$} & $1.7 \mathrm{~s}$ \\
\hline
\end{tabular}

Table 2: Asymptotically quadratic rates of convergence for residual force (left sub-columns) and energy (right sub-columns) during a typical global time step for the two axially uniform G\&R simulations shown in Fig. 2 (c and d) as well as total elapsed CPU time (1+10 time steps each, Fig. 4) using a single processor. Note that both convergence rate and computational time are comparable to those for a hyperelastic computation using the same FE model and increase in pressure without G\&R (not shown). 


\begin{tabular}{|c||c|c|}
\cline { 2 - 3 } \multicolumn{1}{c|}{} & force & energy \\
\hline \hline Iteration 0 & $1.2 \mathrm{E}+00$ & $8.7 \mathrm{E}-01$ \\
Iteration 1 & $1.1 \mathrm{E}-01$ & $3.1 \mathrm{E}-02$ \\
Iteration 2 & $2.5 \mathrm{E}-03$ & $3.5 \mathrm{E}-06$ \\
Iteration 3 & $5.5 \mathrm{E}-06$ & $1.0 \mathrm{E}-10$ \\
Iteration 4 & $2.1 \mathrm{E}-11$ & $2.2 \mathrm{E}-19$ \\
\hline \hline Time (1+10 steps) & \multicolumn{2}{|c|}{11 min $20 \mathrm{~s}$} \\
\hline
\end{tabular}

Table 3: Asymptotically quadratic rates of convergence for residual force (left column) and energy (right column) during a typical global time step for the simulation of a hypertensive asymmetric fusiform aneurysm, with an asymmetric degradation of elastin and uniform increase in pressure, shown in Fig. 8 (mesh e), as well as total elapsed CPU time $(1+10$ time steps) using a single processor. The FE model comprised $N_{r} \times N_{\theta} \times N_{z}=4 \times 160 \times 60=38400$ displacement-based hexahedral elements. 